UC-21

$L A-\sim 9249-T$

DE82 011997

\title{
A Study of Degenerate Four-Wave Mixing in Germanium and Rhenate-Doped Potassium Chloride at Carbon-Dioxide-Laser Wavelengths
}

David Edward Watkins 
CONTENTS

ABSTRACT . . . . . . . . . . . . . . . .

CHAPTER I: Introduction . . . . . . . . . . I

A. Historical perspective of this work....... .

B. A grating picture of DFWM . . . . . . . . .5

C. Synopsis of the thesis . . . . . . . . . .9

CHAPTER II: Experimental Setup for Degenerate

Four-Wave Mixing Studies........ 20

A. The $\mathrm{CO}_{2}$ laser system ........... 20

B. The DFWM experiment... . . . . . . . 26

CHAPTER III: Degenerate Four-Wave Mixing in a

Kerr-Like Medium ........... 33

A. The third-order nonlinear susceptibility

and the four coupled equations for DFWM. . . 35

B. DFWM in the small signal limit . . . . . 46

C. DFWM in the large signal limit ...... . 56

D. Discussion and Summary . . . . . . . 64

CHAPTER IV: Theory of Degenerate Four-Wave Mixing

in Resonantly Enhanced Media..... . 68

A. Theory of DFWM in a two-level homogeneously

broadened saturable absorber ...... . 70

B. DFWM in an inhomogeneously broadened

saturable absorber ........... 79

C. Discussion ............. 82

CHAPTER V: DFWM in P-Type Germanium .......... 91

A. P-type Ge as an inhomogeneously broadened

saturable absorber ........... . . 92

B. Model of DFWM for p-type Ge . . . . . . 97

C. DFWM experiments in p-type Ge....... . 102

D. Discussion and conclusions . . . . . . 109 
CHAPTER VI: DFWM in the Plasma Formation Regime of Ge .119

A. The plasma formation process in germanium . . .121

B. DFWM in the plasma formation regime of $\mathrm{Ge} . . .125$

C. The decay of a laser induced plasma grating in Ge.................133

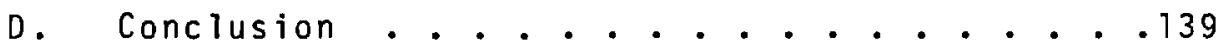

CHAPTER VII: Degenerate Four-Wave Mixing in $\mathrm{KC1} \mathrm{KReO}_{4}$................ 142

A. A description of $\mathrm{KCl}_{1}: \mathrm{KReO}_{4}$. . . . . . . . . 143

B. Polarizability of a three-level system . . . .148

C. Theory of DFWM in KCl:KReO4 ........ . . 157

D. Experimental results in $\mathrm{KCl}: \mathrm{KReO} 4 . . . . .162$

E. Discussion and Conclusions . . . . . . . .174 ChAPTER VIII: Conclusion . . . . . . . . . . 179

A. Review ................ . . . . . . . . 79

B. Directions for future research . . . . . . 182 ACKNOWLEDGEMENTS . . . . . . . . . . . . . . . . . . 185 BIBLIOGRAPHY . . . . . . . . . . . . . . . 186

Appendix A: Determination of the third Order Nonlinear Optical Coefficients of Germanium Through

Ellipse Rotation.......... . . . . . 94

Appendix B: The three-level polarizability in the slowly varying envelope approximation . .203

A. The zero-order polarizability in the slowly varying envelope approximation ..... . 203

B. The first-order polarizability in the SVEA and the derivation of the coupling coefficients. .206 


\section{LIST OF FIGURES}

Number

Page

I-1 Grating formed by the interaction of

two laser beams in a nonlinear medium. . . . 7

II- $1 \quad \mathrm{CO}_{2}$ oscillator-amplifier system . . . . . 21

II-2 DFWM experiment . . . . . . . . . . . . 27

III-1 Geometry for analysis of DFWM . . . . . . . 41

III-2a DFWM in a $3-\mathrm{mm}$ sample of opticai grade, multicrystalline Ge........... . . 51

III-2b DFWM in a 1-cm sample of intrinsic, multicrystalline Ge.......... . . 52

III-2c DFWM in a 2-cm sample of optical grade, single-crystalline Ge... . . . . . . . 53

III-2d DFWM in a 4-cm sampie of intrinsic, single crystalline Ge............. . . 54

III-3a Theoretical reflectivity in the regime of significant pump depletion . . . . . . . 62

I I-3b Comparison of experimental results to theory in the regime of pump depletion . . . 63

IV-1 Comparison of DFWM models with and without pump attenuation by the medium . . . . . 83

IV-2 Comparison of DFWM models as a function of detuning for constant attenuation.... . . 87

IV-3 Comparison of DFWM models for homogeneously and inhomogeneously broadened media.. . . 88

$V-1 \quad V a l a n c e$ band structure of Ge . . . . . . . 93

V-2 Absorption cross-section as a function of wave number in p-type Ge . . . . . . . 95

V-3 Effect of including Drude-zener absorption in modeling saturable absorption in Ge . . 98

V-4 DFWM in a 6-mm, p-type sample of Ge.... . 104 
V-5 DFWM in a $3-\mathrm{mm}, \mathrm{p}$-type sample of Ge... .106

V-6 DFWM in a $1-\mathrm{cm}, \mathrm{p}$-type sample of Ge ....108

V-7 Comparison of the change in index due to thermal changes, bound electrons, and the interband transition .......... . . 114

VI-1 Free-carrier density as a function of $\mathrm{CO}_{2}$ laser intensity in Ge......... . . 126

VI-2 DFWM in two, 3-mm samples of Ge......128

VI-3 Experiment for laser-induced grating decay measurements ............. . . 135

VI-4 Scattering efficiency of a laser-induced grating in Ge as a function of time... . . 137

VII-i Diagram of the Re04 molecule . . . . . . .144

VII-2 Absorption coefficient as a function of photon energy in $\mathrm{KCl}: \mathrm{KReO}_{4}$....... . . 146

VII-3 Saturation of the optical transmission of $\mathrm{KCl}: \mathrm{KReO}_{4}$................ 147

VII-4 Energy diagram of the $v_{3}$ vibrational spectrum of $\mathrm{KCl}: \mathrm{KReO}_{4} . . . . . . . . .149$

VII-5 DFWM on resonance in a $1-\mathrm{cm}$ sample of KC. $1: \mathrm{KReO}_{4}$..................... 164

VII-6 DFWM on resonance in a $2-\mathrm{cm}$ sample of

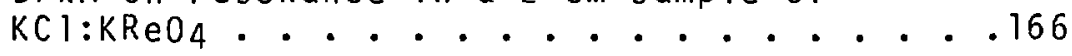

VII-7 DFWM in KCl:KReO4 detuned to the red and to the blue of resonance .........170

VII-8 DFWM in $\mathrm{KCl}: \mathrm{KReO}_{4}$, detuned to the blue of resonance................ . . 171

VII-9 DFWM in KCl:KRe04, detuned to the red of resonance................ . . 173

A-1 Experimental arrangement for measurements of nonlinear susceptibility through ellipse rotation .......................... 196

A-2 Ellipse rotation for two orientations in intrinsic germanium ............. . 198 
A STUDY OF DEGENERATE FOUR-WAVE MIXING IN GERMANIUM AND RHENATE-DOPED POTASSIUM CHLORIDE AT CARBON DIOXIDE LASER WAVELENGTHS

by

David Edward Watkins

ABSTRACT

Theoretical and experimental studies of degenerate four-wave mixing (DFWM) by three different mechanisms are presented. These are the nonlinear index of refraction of a lossless, Kerr-like medium, the saturable absorption of a resonant optical transition, and the formation of a free-carrier grating.

Results of large-signal DFWM experiments in a Kerr-like medium, Ge, provide the first quantitative comparison of experiment to theory in this regime. The mean absolute deviation of the data from a theoretical model with no free parameters is $8 \%$. This deviation can be accounted for by variations in the approximately constant pump intensity.

Theoretical models for small-signal DFWM in saturable absorbers are derived. The novel feature of these models is that pump attenuation by the medium is included. The first experimental results to indicate the importance of pump attenuation effects, taken in $\mathrm{KCl}: \mathrm{KReO}_{4}$, are also presented. 
A unique three-level model for $\mathrm{KCl}: \mathrm{KReO}_{4}$ is derived and used to explain an observed increase in reflectivity at high pump intensity and a frequency-asymmetric behavior in the reflectivity that cannot be explained by existing two-level models.

P-type Ge is used to study the mixture of Kerr-like and saturable absorption effects in DFWM. Experimental results in a lightly doped, 6-mm sample of $p$-Ge agree with a theoretical model which includes both the broadband inhomogeneous saturable absorption and the Kerr-like nonlinearity. Saturable absorption is shown to dominate the interaction below $10 \mathrm{MW} / \mathrm{cm}^{2}$ and the Kerr-like mechanism dominates above this pump intensity.

Free carriers can be induced in Ge by a cascade process, and can be used as a mechanism for DFWM. Amplified reflection by this process has been observed. Both the magnitude and the pump-intensity dependence of the reflectivity in the plasma formation regime have been successfully modeled by calculating the change in index of refraction due to the generation of free carriers. 


\section{CHAPTER I}

INTRODUCTION: A STUDY OF DEGENERATE FOUR-WAVE MIXING IN Ge AND KCI:KReO $\mathrm{K}_{4}$ at $\mathrm{CO}_{2}$-LASER WAVELENGTHS

A. Historical perspective for this work

Early in 1977, Hellwarth proposed the use of degenerate four-wave mixing (DFWM) by nonlinear refraction in a Kerr-like medium to generate optical phase conjugate wavefronts. This paper was followed almost immediately by other papers proposing alternative theories and mechanisms for $D F W M^{2}, 3,4$ and by a host of proposals for the application of phase-conjugate wavefronts. ${ }^{5-12}$ Some of the proposed applications are image reconstruction, 5 narrow optical band pass filters, ${ }^{6}$ differential holography, ${ }^{7}$ spatial convolution and correlation of optical fields, ${ }^{8}$ spatial diffusion measurements, ${ }^{9}$ doppler-free spectroscopy, ${ }^{10}$ self-adjusting laser-target systems, 11 and, more recently, optical bistability. 12 This profusion of possible applications prompts me to quote Mark Twain: 13 "There is something fascinating about science. One gets such wholesome returns of conjecture out of such a trifling investment of fact."

In all fairness, several of these applications have been demonstrated with reasonabe success. For example, a very interesting demonstration of spatial convolution and 
correlation of optical beams by DFWM has been given by White and Yariv. ${ }^{14}$ The reconstruction of the image of a template for ultraviolet photolithography has been demonstrated with $0.5 \mu \mathrm{m}$ resolution by Levenson. ${ }^{15}$ In general, however, no application has seen its way through to acceptance as the best technique for the solution of a practical problem.

Nevertheless, this vision of new technologies has prompted investigation of the DFWM processes by a variety of peopie. I have been among them.

The research I present in this thesis has been supported by the laser fusion division at Los Alamos National Laboratory. The application of interest to Los Alamos is the possibility of automatic target alignment and the removal of phase aberrations for laser-target systems. 11 For this application, an efficient, scalable, nonlinear medium for DFWM at $10 \mu \mathrm{m}$ is needed. My task has been to characterize some candidates for this application.

The production of phase conjugate wavefronts by DFWM is basically the simultaneous writing and reading of a temporary hologram. This thesis concerns itself with the physics of the optical nonlinearities that produce the gratings, or holograms, and the efficiency of these gratings in the generation of new waves. I am not 
concerned with the phase conjugate property of the waves in this thesis. However, since almost all possible applications are concerned with this phase conjugate nature, I develop a prief introductory history to the concepts from this point of view.

In the early stages of the development of holography, the generation of a wavefront proportional to the complex conjugate of the signal wave represented a major source of unwanted noise. ${ }^{16}$ These early electron microscope 17 and $x-r a y^{18}$ holograms were produced by transmission through semitransparent media, with the signal and reference beams collinearly incident on the recording medium. Thus, when read, the reconstructed information consisted of a wave proportional to both the signal wave and its complex conjugate. With the advent of the laser as a high-quality coherent source of optical radiation, optical holograms were produced, and it was quickly realized that the unwanted conjugate portion of the reconstructed wave could be displaced by recording the hologram with the reference beam at an angle to the signal beam. ${ }^{19}$ The conjugate wave could now be neglected, and, for the most part, it was.

The next development to set the stage for phase conjugation via DFWM was the demonstration by Gerritsen ${ }^{20}$ in 1967 of transient or temporary holograms 
in a saturable dye solution. Then, in 1970, Woerdman 21 demonstrated in an imaging experiment the formation of transient free-carrier holograms in Si, using a DFWM geometry and observing the phase conjugate beam. About this same time, Stepanov, Ivakin, and Rubinov 22 demonstrated wavefront conjugation and distortion correction in a four-wave mixing process. Although for the most part this work passed virtually unnoticed in the U. S., investigations continued in the Soviet Union with several theoretical papers discussing various techniques for the production of phase conjugate wavefronts, 23 and several experimental studies of the phase conjugation process in stimulated Brillouin and Raman scattering. 24 Work on transient holograms and other laser-induced grating phenomena received more general attention and is the subject of an excellent review paper by Eichler. 25 It is against this background that Hellwarth, Yariv, and others rediscovered the concept of phase conjugation. In the explosion of papers in the literature since the more recent work of these people, many new ideas have emerged. In developing the ideas in this thesis, I build upon this more recent work. 


\section{B. A grating picture of DFWM}

So far I have described DFWM and phase conjugation as the generation of a temporary hologram. While I confine myself to models for DFWM based on the nonlinear polarizability of various media throughout most of this thesis, the grating picture of DFWM that is the basis of the holographic analogy and the concept of phase conjugation is useful heuristically. In this section, I present a model based on this idea.

By illuminating a standard permanent hologram with a beam propagating in exactly the opposite direction of the original reference beam used to record the hologram, a wave that is proportional to the complex conjugate of the signal wave used to record the hologram is produced. This is directly analogous to phase conjugation by DFWM. The distinguishing characteristics of DFWM are the transient nature of the interaction (from $10^{-12}$ to $1 \mathrm{~s}$ ), the simultaneous "writing" and "reading" of the "hologram," and the additional problems with resolution that arise from the nonlinear nature of the DFWM process.

DFWM is a process where forward and backward counterpropagating pump beams interact with a signal or probe beam in a nonlinear medium. ${ }^{26}$ The result of the interaction is the generation of a fourth wave which is counterpropagating to the signal wave and which, at least 
under certain conditions, is proportional to the complex conjugate of the input signal wave. This process can te viewed as the scattering of one pump beam off a grating generated in the medium by the response of the modium to the other pump wave and the probe wave.

Consider, for example, the forward pump and probe waves as diagrammed in Fig. 1. Assume these waves are both polarized perpendicular to the plane of the page. The interference pattern generated by these two waves are planes of maxima and minima which bisect the angle between the waves, as shown in the figure. If I write the pump wave as $E_{f} \exp \left[i\left(k_{z} z+k_{x} x\right)\right]$ and the probe wave as $E_{1} \exp \left[i\left(k_{z} z-k_{x} x\right)\right]$ then the interference pattern can be described by the difference in the wave vectors of the pump and probe,

$$
\vec{k}_{g}=\left(k_{z} \hat{z}+k_{x} \hat{x}\right)-\left(k_{z} \hat{z}-k_{x} \hat{x}\right)=2 k_{x} \hat{x}
$$

If the medium responds in a nonlinear fashion to the electric fields of the intensity pattern set up by the pump and probe, say with an intensity-dependent index of refraction, the response will set up a grating, in this case a phase grating. This grating will exhibit the same variations as the intensity interference pattern of the 


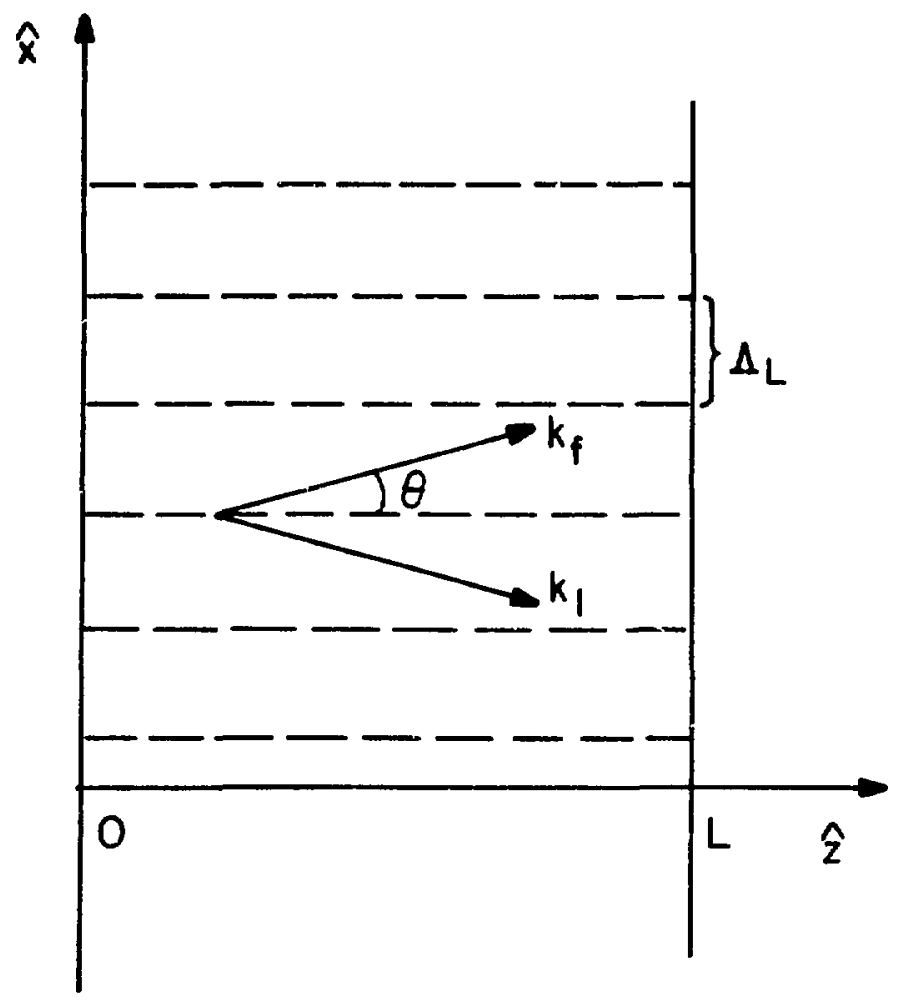

Figure I-1. The grating formed by the interaction of two laser beams of equal frequency in a nonlinear medium. The wave vectors of the two beams are $k_{f}$ and $k_{1}$ and the angle between them is $2 \theta$. The period of the interference pattern is $\Lambda_{L}$. If the medium responds in a nonlinear fashion, a grating is set up. A wave of equal frequency propagating opposite to $k_{f}$ (i.e. the backward pump beam) is incident on the grating at the Bragg angle and is diffracted, creating a beam counterpropagating to the signal kl. 
waves. Thus it has a period

(2) $\quad \Lambda_{L}=\frac{2 \pi}{k_{g}}=\frac{\pi}{k_{x}}=\frac{\lambda}{2 \sin (\theta)}$

where $\lambda$ is the optical wavelength in the medium and $\theta$ is the half angle between the pump and the probe.

The grating formed in the mediuin scatters the backward pump beam. This beam is taken as $E_{b} \exp \left[-i\left(k_{z} z+k_{x} x\right)\right]$. The possible directions for scattering are given by adding integral multiples of the graiting wave vector, $\mathrm{K}_{\mathrm{g}}$, to the wave vector of the backward wave. The only nonzero integral multiple of $\mathrm{K}_{\mathrm{g}}$ which satisfies the bragg condition when added to the backward wave vector is $m=1$. This gives the wave vector of the diffracted beam as $-k_{z} \hat{z}+k_{x} \hat{x}$, counterpropagating to the initial probe wave.

The initial probe wave also interferes with the backward pump beam to generate a grating of period

$$
\Lambda_{s}=\frac{\pi}{k_{z}}=\frac{\lambda}{2 \cos (\theta)}
$$

This grating scatters the forward pump wave into the backward direction of the initial probe wave. Because the angle $\theta$ is generally small, $\Lambda_{L}$ denotes the graiting with larger spacing and $\Lambda_{S}$ is the small-spaced grating.

Finally, I note that it is not necessary trat the polarization of the probe beam be the same as that of the 
pump beam. Perpendicular polarizations can create the same type of grating. That this is the case can be understood by analogy of a nonlinear medium, say a Kerr-like medium, with a mass held in a plane by four springs. The oscillation of the mass along one direction can stretch the perpendicular springs beyond the Hooke's law regime for these springs, introducing a nonlinear behavior. Thus the response of the mass to an oscillatory force in one direction can be dependent on the oscillatory force in the perpendicular direction, and on the relative phase of this perpendicular oscillation. A Kerr-like medium responds the same way, and thus it is not necessary for the probe wave to be polarized in the same direction as the pump wave.

\section{Synopsis of the thesis}

The purpose of this thesis is to develop theoretical models for DFWM and to compare these models to experimental results for DFWM in germanium and in an impurity doped alkali halide, $\mathrm{KCl}: \mathrm{KF}_{\mathrm{eO}} \mathrm{H}$. The point of comparison is the reflectivity, or the ratio of the intensity of the generated wave to the intensity of the signal wave. No attempt is made to predict or to measure quantitatively the quality of the generated wave (as the phase conjugate of the signal, for example). Three different mechanisms 
for DFWM are investigated; the nonlinear index of refraction of a lossless, Kerr-like medium, the saturable absorption of a resonant optical transition, and the formation of an optically induced free-carrier grating in a semiconductor.

The problems that this thesis addresses are as follows:

1. What is the effect of pump depletion by the four-wave mixing piocess in a lossless medium on the reflectivity, both theoretically and experimental1y?

2. What is the effect of pump attenuation by the medium (that is, absorption as distinguished from wave-to-wave conversion) on DFWM in saturable absorbers?

3. Given our knowledge of the saturable absorption and the Kerr-like nonlinearity of p-type Ge, can a comprehensive model be developed which explains the observed reflectivity in this material?

4. Is it possible to characterize the DFWM in the free-carrier formation regime of $G e$ by the index associated with the plasma?

5. Can a three-level model for $K C 1: K R e O_{4}$ be developed that can describe those features of the observed reflectivity in this material which cannot be explained by existing two-level models? 
In the remainder of this section, a brief synopsis of each chapter is presented as it related to the above problems.

The second chapter deals with the experimental facility and the techniques for DFWM I use throughout the thesis.

Chapter III is a study of DFWM in lossless Kerr-like media. This study is motivated by the amenability of such media to analysis, as well as their popularity in experiments. Analytic solutions for the reflectivity in DFWM are now available for both the small signal and the large-signal cases. 26,27 Understanding the mechanisms involved in DFWM in lossless media allows one to extrapolate certain physical processes to absorbing media, and, more specifically for p-type Ge, may be fundamental to understanding the mixture of resonantly enhanced and nonresonant DFWM processes.

My experimental results for DFWM in a Kerr-like medium, intrinsic Ge, demonstrate the validity of the published small-signal theory ${ }^{27}$ and are in good agreement with my original measurement of the third-order nonlinear susceptioility of Ge (see Appendix A). These results also demonstrate the failure of the time independent, small signal theory to quantitatively predict the magnitude of the wave mixing if the laser pulse is short compared to the optical path length in the medium. 
In Chapter III, I also present a numerical technique for the solution of the four coupled differential equations which describe DFWM in Kerr-like media in the large signal limit. I have obtained the only experimental results for DFWM in the large signal limit, and compare these results to the nurierical model. Although these results are in good agreement with the numerical model, it is not clear that the results could discriminate between the numerical model and a less complete aralytical solution of the coupled differential equations given by Hsu. ${ }^{28}$ The study of the large signal limit for DFWM was partially motivated by the incomplete solution obtained by Hsu. Since I developed the numerical solution, a complete but cumbersome analytic solution for the large signal case has been given by winful. 29 The relative merits of these models are discussed in Chapter III.

In Chapter IV, I expand the theory of DFWM in a two-level saturable absorber given by Abrams and Lind. ${ }^{3}$ The result is a numerical technique for the solution of the generalized coupled differential equations which describe the propagation of the pump and probe waves in DFWM in the small-signal limit. The motivation for such a study is multifaceted. The first good experimental results I obtained for DFWM were in $K C 1: K R e 0_{4}$, which is a saturable absorber. These results demonstrated the need 
to modify the Abrams and Lind model for DFWM in such media ${ }^{3}$ to incorporate the effect of attenuation of the pump beams by the medium. Also, the published model describes DFWM in homogeneously broadened media. My interest in p-type Ge, which saturates inhomogeneously, necessitates further modifications to the theory. The results of this chapter provide a background for the models for DFWM given in Chapters $V$ and VII.

Chapter $V$ extends the results of Chapters III and IV to obtain a model for DFWM in p-type Ge. The study of DFWM in p-type Ge is of interest because Ge has a large inherent nonlinear index of refraction, $p$-type Ge has an additional nonlinearity aue to saturable absorption, and Ge is available in large simples. These features make Ge an attractive candidate for DFWM experiments and applications. My interest in this chapter is primarily in the mixture of effects in DFWM arising from the inherent nonlinear index of refraction and the saturable absorption. I derive the first comprehensive model for DFWM in this material and compare it to experimental results. The comparison is good in the limit of wcak absorption. Various mechanisms that introduce additional nonlinearities are discussed, but no explanation is found for the observed discrepancy between the model and the experimental results in strongly absorbing samples. 
The investigation of DFWM at high pump intensities in the plasma formation regime of Ge presented in Chapter VI is prompted by the serendipitous experimental observation of a rapid and dramatic increase in the wave mixing process over a small range in intensity near the surface damage threshold of Ge. Since the original observation, the wave mixing process has been identified as being due to the generation of free carriers by nonlinear optical absorption. Results in various samples of Ge are discussed, and a model is derived which successfully predicts the magnitude of the wave mixing process and its intensity dependence. In a second experiment prompted by these results, the decay of a laser-incluced, free-carrier grating of the type which is responsible for the enhanced DFWM has been observed. The results of this experimeni are correlated with a diffusion coefficient identical in magnitude to the ambipolar diffusion coefficient in Ge at room temperature, and confirm our expectation that the free carriers are rapidly thermalized.

Finally, in Chapter VII, I deal with DFWM in $K C 1: K_{R} 0_{l}$. The original motivation for the study of DFWM in this material was that it acts like an ideal, two-level, homogeneously broadened medium in saturable absorption experiments. 30 Therefore, it was thought to be an ideal candidate for comparison to the model given by 
Abrams and Lind. 3 In fact, my early experimental investigations of $\mathrm{DFWM}$ in $\mathrm{KCl}: \mathrm{KReO}_{4}$ were the motivation for the study presented in chapter IV. In addition to pointing out the need for including pump attenuation effects in modeis of DFWM in saturable absorbers, there was a strong indication in my data that a multilevel model might be necessary to adequately describe DFWM in this material. Even so, the spectroscopy of $\mathrm{KCl}: \mathrm{KReO}_{4}$ was thought to be relatively simple compared to other infrared saturable absorbers (for instance, $\mathrm{SF}_{6}$, which has also been studied extensively $y^{31}$, and thus might be a good candidate for the study of multilevel effects. If a simple three-level model could describe this material, it might be possible to extend the results to more complicated media.

I have developed a model for the polarizability of $K C l: K R e 0_{4}$, treating the medium as a three-level system, with the third level acting as a perturbation on the lower two levels. This unique model for the polarizability has been applied to the description of DFWM in this material. The model describes DFWM on resonance well for weakly absorbing samples. It also works well away from the single-photon resonance. On resonance in strongly absorbing media, qualitative agreement is obtained, but the model does not quantitatively describe the DFWM in such a sample. 


\section{Footnotes}

1. R. W. Hellwarth, "Generation of time-reversed wive fronts by nonlinear refraction, "Journal of th: Optical Society of America 67, 1 (1977).

2. A. Yariv and D. M. Pepper, "Amplified reflection, phase conjugation, and oscillation in degenerate four-wave mixing," Optics Letters 1, 16 (1977).

3. R. L. Abrams and R. C. Lind, "Degenerate four-wave mixing in absorbing media," Optics Letters 2,94 (1978) and Erratum, optics Letters 3, 205 (1978).

4. J. H. Marburger and J. F. Lam, "Effect of nonlinear index changes on degenerate four-wave mixing, "Applied Physics Letters 35,249 (1979).

5. Image reconstruction by DFWM was first recognized by Woerdman (Ref. 2l), but is also fundamental to the work of Hellwarth (Ref. 1).

6. D. M. Pepper and R. L. Abrams, "Narrow optical bandpass filter via nearly degenerate four-wave mixing," Optics Letters $\underline{3}, 2$ (1979).

7. A. E. Siegman, "Dynamic interferoretry and differential holography of irregular phase objects using phase conjugate reflection," Optics Communications 31,257 (1979).

8. D. M. Pepper, J. Auyeung, D. Fekete, and A. Yariv, "Spatial convolution and correlation of optical fields via degenerate four-wive mixing," Optics Letters 3,7 $(1978)$.

9. D. S. Hamilton, D. Heiman, J. Feinberg, and R. W. Hellwarth, "Spatial diffusion measurements in impurity doped solids by degenerate four-wave mixing," Optics Letters 4, 124 (1979).

10. D. C. Haueisen, "Doppler-free two-photon spectrosccpy using degenerate four-wave mixing, "Optics Communications $28,, 183(1979)$.

11. Yu. I. Kruzhilin, "Self-adjusting laser-target system for laser fusion," Soviet Journal of Quantum Electronics 8,359 (1978), and I. Liberman, "Application of phase conjugation to $\mathrm{CO}_{2}$ lasers," in Proceedings of the Los Alamos Conference on Optics 179, D. H. Liebenberg, Ed. (SPIE, Washington 1979), p. 426. 
12. H. G. Winful, "Optical bistability in periodic structures and in degenerate four-wave mixing, "in the Digest of Technical Papers, International Conference on Excited States and Multiresonant Nonlinear Optical Processes in Solids, D. S. Chemla, Ed. (Les Editions de Physique, Orsay, France, 1981), p. 55.

13. Mark Twain, Life on the Mississippi (Bantam Books, Inc. New York, 1972) p. 93.

14. J. 0. White and A. Yariv, "Real-time image processing via four-wave mixing in a photorefractive medium," Applied Physics Letters 37, 5 (1980).

15. M. D. Levenson and K. Chiang, "Application of phase conjugation to photclithography, "presented at the Conference on Lasers and Electro-0ptics 181

(Washington, D. C., 10-12 June 1981).

16. H. M. Smith, Principles of Holography (John Wiley and Sons, New York, 1975), p. 3.

17. D. Gabor, "Microscopy by reconstructed wavefronts," Proceedings of the Royal Society, Series A, 197, 454 $(1949)$.

18. H. M. A. El Sum, Reconstruction Wavefront Microscopy, (Ph.D. thesis, Stanford University, T952).

19. E. M. Leith and J. Upatnieks, "Reconstructed wavefronts and communication theory," Journal of the Optical Society of America 52, 1123 (1962).

20. H. J. Gerritsen, "Nonlinear effects in image formation," Applied Physics Letters 10, 237 (1967).

21. J. P. Woerdman, "Formation of a transient free-carrier hologram in Si," Optics Communications 2, 212 (1971).

22. B. I. Stepanov, E. V. Ivakin, and A. S. Rubinov, "On registration of plane and volume dynamic holograms in bleachable substances," Doklady Akad, Nank. SSSR 196, 567 (1971).

23. For examples, see Yu. A. Anan'ev, and A. Urbanovich, "Mechanism of diffraction of light by optically induced gratings in absorbing media," Soviet Journal of Quantum Electronics $5,1320(1976)$ and P. Yu. Zel'dovich, V. I. Popovichev, V. V. Ragul'skii, and 
F. S. Faizullov, "Connection between the wavefronts of the reflected and exciting light in stimulated

Mandel'shtam-Brillouin scattering," Soviet Physics JETP 15, 109 (1972).

24. For examples see Yu. Ostrovskii, V. G. Sidorovich, D. I. Stastl'ko, and L. V. Tarrin, "Dynamic holograms in sodium vapor," Soviet Technical Physics Letters 1, $442(1975)$, E. V. Ivakin, I. P. Petrovich, and A. S. Rubinov, "Self diffraction of radiation by light induced phase gratings," Soviet Journal of Quantum Electronics $3,52(1372)$. and E. V. Ivakin, I. P. Petrovich, A. S. Rubinov, and B. I. Stepanov, "Dynamic holograms in an amplifying medium, "Soviet Journal of Quantum Electronics $\underline{5}, 840$ (1975).

25. H. J. Eichler, "Laser induced grating phenomena," Optica Acta 24, 631 (1977).

26. Throughout the thesis, when discussing DFWM, the four waves are divided into two classes, the pump waves and the probe waves. The pump waves are denoted "forward" or "backward" depending on their direction of propagation relative to the $z$-axis. The probe waves are denoted "signal" (in analogy to the holographic terminology) for the experimenter-controlled input wave and "generated" for the wave produced in the interaction. A slight variation of this terminology is used in section $C$ of Chapter VI, where I discuss the decay of a laser induced grating.

27. Small signal solutions are given in Ref. 1,2 , and 4 . The most complete solution is given in Ref. 4 .

28. H. Hsu, "Large-signal theory of phase conjugate backscatterings," Applied Physics Letters 34,855 (1979).

29. For the only complete solution for the iarge signal case see Ref. 12. Other attempts at solutions which are incomplete are given by Hsu (Ref. 28) and by J. H. Marburger and J. F. Lam, "Nonlinear theory of degenerate four-wave mixing, "Applied Physics Letters 34, $389(1979)$.

30. R. K. Ahrenkiel, D. J. Dunlavy, and A. J. Sievers, "A new class of saturable absorbers at $10.6 \mu \mathrm{m}$ based on doped alkali halides," IEEE Journal of Quantum Electronics QE-16, 225 (1980). 
31. D. G. Steel and J.F. Lam, "Multiline phase conjugation in resonant materials," Optics Letters 5 , $297(1980)$. 
CHAPTER II

EXPERIMENTAL SETUP FOR DEGENERATE FOUR-WAVE MIXING STUDIES

Introduction

In this chapter I describe the equipment and experimental arrangement used for DFWM experiments throughout the thesis. In Section A, I discuss the $\mathrm{CO}_{2}$ laser system and the changes $I$ made in it to insure safe operation for DFWM experiments. In Section B, I discuss the OFWM experiment itself and some of the data reduction technique.

\section{A. The $\mathrm{CO}_{2}$ laser system}

The $\mathrm{CO}_{2}$ laser I use in my studies is an oscillatoramplifier system assembled by Thomas, Harrison and myself in 1977. This laser is illustrated in Fig. 1. The study of DFWM at $\mathrm{CO}_{2}-1$ aser wavelengths $(9-11 \mu \mathrm{m})$ is primarily motivated by the application of DFWM to laser fusion systems for automatic target alignment and the removal of phase aberrations. The optical energy (several Joules) and the intensity (several $\mathrm{GW} / \mathrm{cm}^{2}$ in a $1-\mathrm{cm}$ diameter beam) available from $\mathrm{CO}_{2}$ lasers of moderate size make this laser an attractive candidate for investigation of nonlinear optical phenomena. 


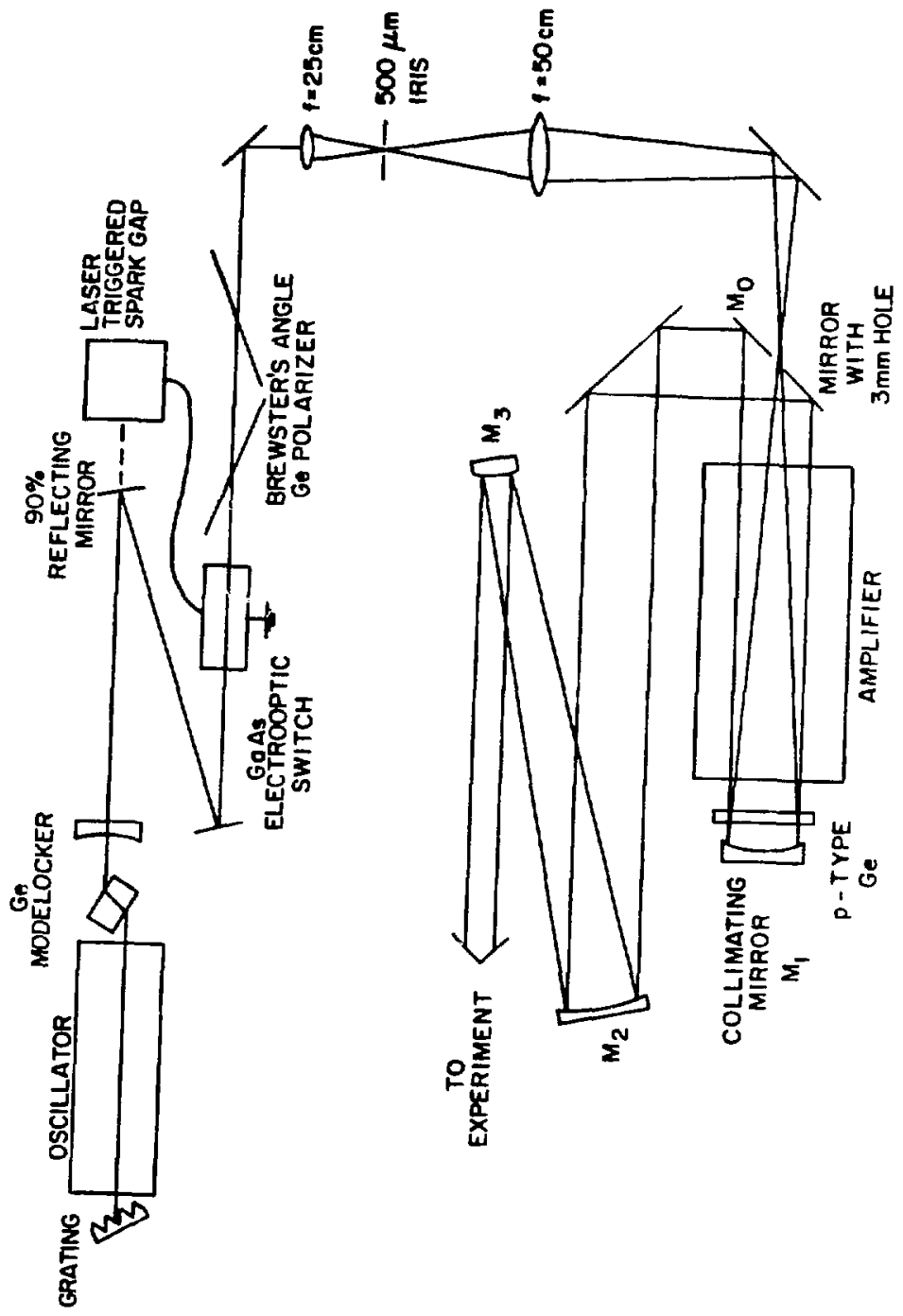

Figure II-1. A $\mathrm{CO}_{2}$ oscillator-amplifier laser system. The oscillator produces a train of 1 to 2 -ns pulses. A single pulse is selected with an electrooptic switch and double passed through an amplifier. A spatial filter with a $500-\mu m$ iris is used for retropulse isolation and a piece of p-type Ge is used to prevent self oscillation by the amplifier and to reduce the background optical energy emitted by the system. The laser is grating controlled and operates on a single, tunable line of $\mathrm{CO}_{2}$. The output on P-20 of the 10-um band is a 1-to 2-J, 1- to $2-n s$ laser pulse. 
The oscillator is a photo-preionized transverseelectricaliy excited medium at atmospheric pressure, with 1-cm aperture and a $90-\mathrm{cm}-10 n g \mathrm{gain}$ length. This oscillator is actively modelocked by an acoustically driven piece of Ge which is inserted into the laser cavity at Brewster's angle. The laser produces a train of TEM $_{00}$ pulses of 1- to 2-ns duration with 12-ns interval having a total energy of about $50 \mathrm{~mJ}$. Most of the energy is in the eight biggest pulses. The oscillator is grating tuned and may be operated on any of several of the $R$ and $P$ lines of both the 9 - and $10-\mu \mathrm{m}$ bands in $\mathrm{CO}_{2} \cdot 1$

The train of laser pulses initates a laser-triggered spark gap. This device generates a $12 \mathrm{kV}$ pulse of about 10-ns duration, which drives an $8 \times 8 \times 60 \mathrm{~mm} \mathrm{GaAs}$ electrooptic switch. The spark gap is timed to activate the switch when the largest pulse of the train is present, rotating the polarization plane of this pulse alone by $90^{\circ}$. A polarizer can then be used to transmit only this single pulse, rejecting the rest of the pulse train. The single pulse energy is typically 2 to $5 \mathrm{~mJ}$.

In the original design of this laser, this pulse was focused through a hole in mirror $M_{0}$ by a single lens and double passed through an amplifier. The amplifier is the same type of medium as the oscillator, but with $3.5-\mathrm{cm}$ aperture and $130-\mathrm{cm}$ gain length. The amplified beam is 
extracted by the mirror $M_{0}$. The diameter of this beam is reduced by a telescope (mirrors $M_{2}$ and $M_{3}$ ) and relayed to the experimental area. The telescope is carefully adjusted to fully utilize the relay to provide a region of maximum brightness and minimum phase curvature at the experiment. The experimental area is located within a "screen room," away from the electrical noise produced by the laser discharges.

For UFWM studies, some modifications to the laser have been necessary. These are primarily related to the presence of the counterpropagating pump beams. Any scheme used to generate these beams produces a retrodirected pulse, ${ }^{2}$ which, after further amplification, can optically damage components between the amplifier and the oscillator. In the present case, the most sensitive element to optical damage is the GaAs electrooptic switch. A related problem arising from this situation is that the high gain amplifier medium is susceptible to self-oscillation.

To avoid these problems, two additional components are necessary in the laser train. The first problem is avoided by replacing the single lens which focused the oscillator pulse into the hole in the mirror with a spatial filter. 
A spatial filter operated in air can be made to break down at $\mathrm{CO}_{2}-1$ aser fluxes of several joules per $\mathrm{cm}^{2}$ in the focal regime, for ns-duration pulses. The plasma formed at the focus blocks the transmission of any 10-um light. Such a device can be designed to transmit the weak forward-going oscitiator pulse and Tater limit the transmission of the amplified retropulse to energies which are safe for sensitive components. 3

The system I designed uses a $25-\mathrm{cm}$ focal length lens which focuses the oscillator pulse through a $500 \mu \mathrm{m}$ iris. The expanding beam from this iris is refocused by a $50-\mathrm{cm}$ focal length lens through the hole in $M_{0}$ in exactly the same way as previously. This spatial filter is designed to break down at an energy of about $5 \mathrm{~mJ}$ in the focal region. The measured maximum transmission of the filter is actualiy about 8 to $10 \mathrm{~mJ}$, even at input energies of over $1 \mathrm{~J}$. This level of isolation is quite adequate to prevent optical damage to sensitive components.

The second addition to the laser system is a piece of p-type Ge placed at the recollimating mirror $M_{2}$. This piece of Ge has a small-signal transmission of $10 \%$ at 10.6 $\mu \mathrm{m}$, and bleaches to higher transmission in the presence of intense $\mathrm{CO}_{2}$-laser radiation. ${ }^{4}$ This acts to reduce the gain of the amplifier system for weak signals, while maintaining a high gain for intense 
signals, and can thus prevent self oscillation of the amplifier system without severe penalty in overall laser performance.

All additional benefit of the insertion of the $p$-type Ge is a reduction of the amount of background light in the laser pulse due to finite optical contrast in the electrooptic switch and modelocker. Insertion of the Ge reduced the background energy from $8 \%$ to 1 ess than $1 \%$ of the total energy, below the $3 \%$ resolution of my calorimeter.

An additional spatial filter is sometimes used between the amplifier and the experiment. This device was originally intended to be the only isolation in the experiment. The unit is evacuated to enhance its power-handling capability. Unwanted spatial frequencies in the laser beam strike the iris, producing a metallic plasma which reduces the transmission of the spatial filter. This level of isolation was not by itself sufficient for my experiments.

The complete laser system produces a 1 - 2-ns pulse with total energy of over $1 \mathrm{~J}$ on the $\mathrm{P}-20$ line of the 10- $\mu \mathrm{m}$ branch of $\mathrm{CO}_{2}$. A small energy penalty is paid for tuning to other lines. The wavefront quality in the experimental region is nearly perfect. 


\section{B. The DFWM experiment}

Figure 2 shows the experimental arrangement used for al1 DFWM experiments presented in this thesis. The goal of the DFWM experiment is to measure the intensity of the generated wave as a function of the pump intensities and the input signal intensity for a given sample. (Usually, but not always, the signal intensity is held constant.) From this measurement, the reflectivity, or ratio of the generated intensity to the input signal intensity, can be calculated and compared to theoretical models. A detailed description of the experiment follows.

The $\mathrm{CO}_{2}$ laser beam is first split to provide a signal for the input monitor system. This consists of a calorimeter which determines the input beam energy with $\sim 20 \%$ absolute accuracy and $3 \%$ resolution, and a pyroelectric detector used in conjunction with an oscilloscope to monitor the temporal profile of the laser pulse. This monitoring system is calibrated to give intensities for all beams in the experiment.

A second splitter is used to obtain a signal or probe beam. Both splitters are, in fact, the front and back surfaces of a single, wedged ZnSe splitter. Neither surface of the ZnSe is optically coated, so at small angle of incidence each split removes 15 to $20 \%$ of the 1 aser energy. ZnSe is used so that a high quality optical 


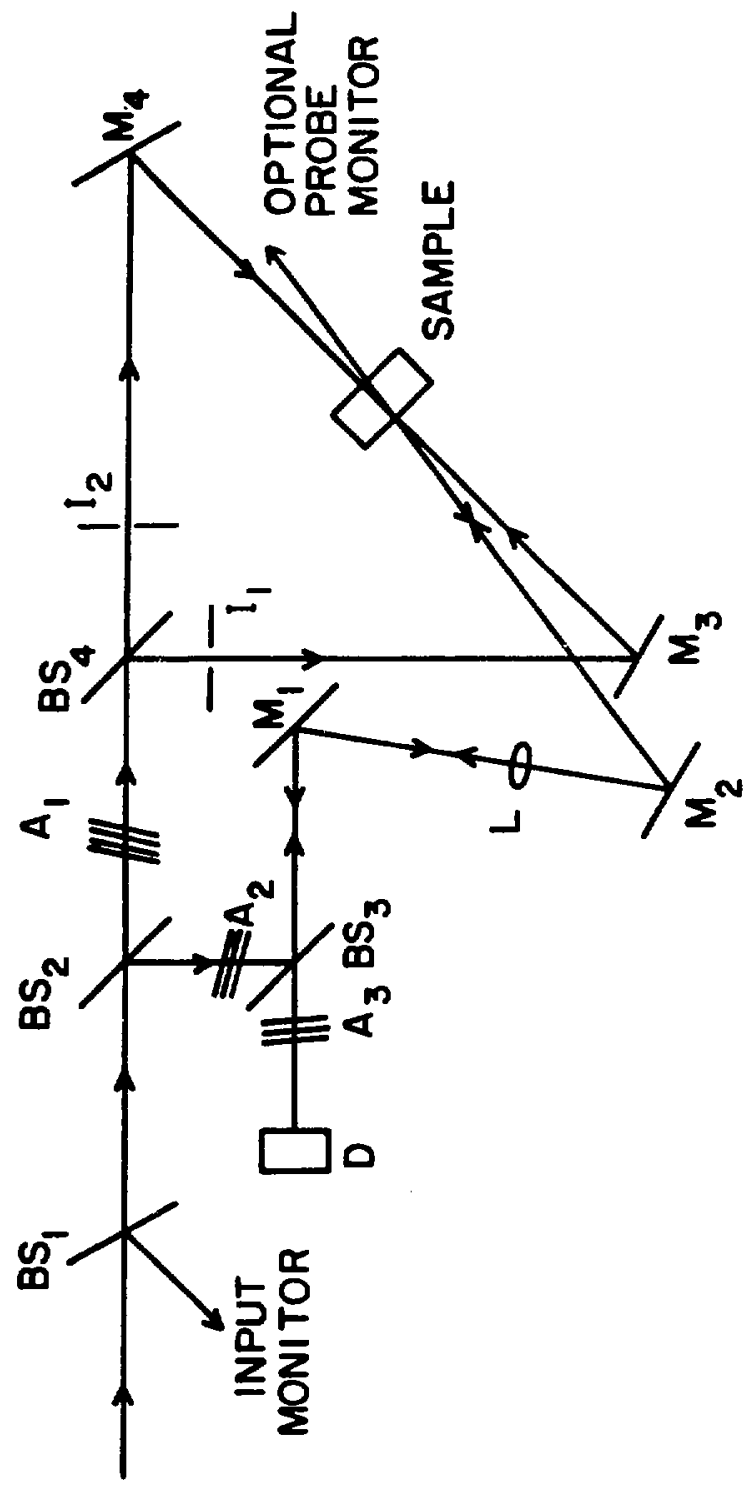

Figure II-2. The DFWM experiment. Beamsplitters BS 1 and $\mathrm{BS}_{2}$ are $15-20 \%$ reflecting. $\mathrm{BS}_{3}$ is about $35 \%$ reflecting and $B_{4}$ is a $50-50$ splitter. The $A_{j}$ are variable $\mathrm{CaF}_{2}$ attenuators used to control intensities. The lens $L$ is a 60 or $76-\mathrm{cm}$ focal length lens which creates a focal spot in the probe beam at the sample. Irises $I_{1}$ and $I_{2}$ are used in conjunction with mirrors $M_{3}$ and $M_{4}$ to align the counterpropagating pump beams. The wave mixing is observed with a Ge:Hg detector at $D$. 
finish can be obtained. In this case, all the optics are $\lambda / 20$. The signal beam is reflected off a third splitter and focused onto the sample.

A fourth splitter is used to obtain the counterpropagating pump beams. This splitter is a piece of $\mathrm{Ge}$, uncoated on the first surface and antireflection coated on the second surface. This gives a $50 \%$ reflection for p-polarized light at about $45^{\circ}$ angle of incidence.

The intensities of the pump beams and the signal beam are controlled by means of calibrated $\mathrm{CaF}_{2}$ attenuators at $A_{1}$ and $A_{2}$ respectively. The intensity in each beam is calibrated to the input monitor by carefully aligning a 1.5-mm-diameter iris in each beam at the sample and measuring the energy transmitted by this iris as a function of the input energy and the attenuation. This information, along with the temporal profile provided by the input pyroelectric detector, gives the intensity in each beam. The signal beam is calibrated at the sample region in the focal spot of the lens $L$.

The lens $L$ is used to focus the signal beam to about $10 \%$ of the diameter of the pump beams. In this way the signal samples a region where the pump beams are fairly uniform in space. This lens also collects the generated wave from the four-wave mixing process, returning it through the third beam splitter and into a detector. 
The intensity at the wave mixing detector $D$ is maintained at a constant level by use of $\mathrm{CaF}_{2}$ attenuators at $C$. This detector is calibrated by placing a mirror at the focal spot of the lens $L$, and carefully aligning the sample beam back on itself. This provides a reference for $100 \%$ reflection, which is calibrated against the input monitor. By maintaining an approximately constant intensity at the detector $D$, any nonlinear response of the detector is avoided.

For the experiments presented in this thesis, the detector $D$ is a liquid-helium cooled Hg-doped Ge device. This detector has the advantage of having about $10^{5}$ times the sensitivity of a pyroelectric detector, such as is used for the input monitor, with only a little more than an order of magnitude loss in response time. The sensitivity is required for measuring extremely weak pulses. Typically, probe intensities must be below the saturation intensity of a saturable absorber. The materials studied here have saturation intensities of over $1 \mathrm{MW} / \mathrm{cm}^{2}$. The required signal intensity is thus about $0.3 \mathrm{MW} / \mathrm{cm}^{2}$, or about $2.5 \mu \mathrm{J}$ in the focused probe beam. To measure a DFWM reflectivity of $10^{-4}$, I must be about to detect a fraction of a $n J$. This restricts my choice of detectors. 
The detector $D$ should also have a fast response time, so it can follow the ns pulses created by the wave mixing. The risetime of the detector employed is on the order of the laser pulse risetime, and the falltime is slightly longer. Thus the actual response of the detection system is some convolution of these behaviors. I ignore this, and simply take the detector response as a measure of the reflectivity. This procedure is a smali source of error in the measurement of absolute reflectivities.

To align the pump beams, irises are carefully centered on the two pump beams near the fourth beamsplitter. The mirror $M_{3}$ is used to align the forward pump beam onto the backward pump beam aperture, and vice-versa for the backward pump beam. This process is iterated until both beams are properly aligned with the sample in place. Then the signal beam can be aligned onto the center of the pump beams at the sample by adjusting mirror $M_{2}$. It is estimated that this technique allows the pump beams to be aligned to within $1 \mathrm{mR}$ of true counterpropagation. Typically, an angle of $15^{\circ}$ is set between the pump and the probe beams, external to the sample. Care is taken to insure that the two pump beams and the signal beam all arrive at the sample within \pm 20 ps of simultaneity.

The optional signal monitor is only used in this work 
to monitor the signal in the presence of plasma formation in Ge. This detector system consists of a pyroelectric detector and a fast, 5-GHz oscilloscope. Only in the case of plasma formation in Ge is the amplification of the forward beam by DFWM significant enough to be observed.

The data obtained from DFWM experiments consists of a chart recording of the input calorimeter and photographs of oscilloscope traces for the input pyroelectric and the DFWM detector. The oscilloscope traces are analyzed by digitizing them with a small computer. A complete pulse analysis is then performed by the computer, giving pulse height (or intensity), risetime, falltime, full width at half maximum, and pulse area. This information can be used to obtain the intensities of all four beams in the experiment.

A typical measurement consists of varying the pump intensity by varying the attenuation at $A_{1}$, while maintaining a constant signal intensity. The result of such an experiment is a measurement of the DFWM reflectivity as a function of pump intensity. 


\section{Footnotes}

1. For a discussion of $\mathrm{CO}_{2}$ spectroscopy, see Handbook of Lasers, R. J. Pressley, Ed. (CRC Press, Inc.

CTeveland, 1977), p. 302 .

2. For an example of an experiment for DFWM, see Fig. 2 . Some experimenters have derived counterpropagating pump beams by simply retroaligning a single beam with a single mirror, in which case the possibility of returning $100 \%$ of the pump beam back into the laser is obvious. In Fig. 2 it is not as obvious, but with some reflection by the reader it is apparent that this system also can return $100 \%$ of the pump beam back to the laser.

3. J. F. Figueira, S. J. Czuchlewski, C. R. Phipps, Jr., and S. J. Thomas, "Plasma-breakdown retropulse isolators for the infrared, "Applied Optics 20, 838 (1981).

4. See Chapter IV, or C. R. Phipps, Jr., and S. J. Thomas, "Saturation behavior of p-type germanium at $\mathrm{CO}_{2}$ laser wavelengths," Optics Letters I, 93 (1977) 
CHAPTER III

DEGENERATE FOUR-WAVE MIXING IN A KERR-LIKE MEDIUM

Introduction

In the presence of the electric field,

$$
\vec{E}(r, t)=\frac{1}{2} \sum_{v_{j}}\left[\vec{E}\left(v_{j}\right) \exp \left(-i v_{j} t\right)+c . c \cdot\right],
$$

the polarizability of a medium can be written as the sum

$$
\left.\vec{P}(r, t)=\frac{1}{2} \sum_{v} \overrightarrow{[P}(v) \exp (-i v t)+c \cdot c \cdot\right] .
$$

In conventionai electromagnetic wave theory, the Fourier component of the polarizability is given by $P_{i}(v)=$ $x^{i j}(\nu) E_{j}(\nu)$, where $x^{i j}$ is the susceptibility and $i$ and $j$ refer to cartesian coordinates. The polarizability can be generalized to include higher order terms in the electric field. That is, the Fourier component of the polarizability at the frequency $\nu$ can be expanded in a series in the applied electric field as ${ }^{1}$

$$
\begin{aligned}
P_{i}(v)= & x_{1}^{i j}(v ; v) E_{j}(v)+x_{2}^{i j k}\left(v ; v_{1}, v_{2}\right) E_{j}\left(v_{1}\right) E_{k}\left(v_{2}\right) \\
& +x_{3}^{i j k 1}\left(v ; v_{1}, v_{2}, v_{3}\right) E_{j}\left(v_{1}\right) E_{k}\left(v_{2}\right) E_{1}\left(v_{3}\right)+\ldots
\end{aligned}
$$


Here, $x_{n}$ is the nth-order susceptibility and $v=v_{1}+v_{2}+\ldots+v_{n}$ for the $n t h$ order.

Summations are taken over repeated indices. If all $v_{i}$ and possible combinations of $v_{i}$ are far from resonance, then $x_{n}$ is real. If this is the case for $x_{3}$, and $x_{2}$ vanishes, the material is termed "Kerrlike," because this nonlinearity is responsible for both the dc and the optical Kerr effect. I note that even orders of the susceptibility vanish in media with inversion symmetry. 2

In typical, transparent, solid-state, Kerr-like media, the first-order susceptibility ranges from about 0.2 to 2 esu, while the third-order susceptibility ranges from $10^{-10}$ to $10^{-15}$ esu. Higher order terms are generally negligible. For example, in intrinsic germanium, $x_{1}=$ 1.19 esu and $x_{3} \simeq 3 \times 10^{-11}$ esu. No higher order effect has been identified in Ge. The susceptibilities can be greatly enhanced by the presence of resonances. In this chapter, I will deal with effects due to the third-order term. It is this effect that was first proposed as a mechanism for DFWM in the papers that sparked the intense interest of the last few years. This third-order term has received much attention since the early days of the study of nonlinear optics.

The novel features of DFWM in Kerr-like media presented here are as follows: A quantitative comparison 
is made between the third-order nonlinear susceptibility as deduced from DFWM experiments and the time-independent theory to previous measurements of $x_{3}$ (see Appendix A). I also present a numerical technique for the solution of the coupled differential equations which describe DFWM in the large signal limit and compare experimental DFWM data to this model.

Chapter III is organized as follows: In the first section I discuss the third-order nonlinear susceptibility and the derivation of the four coupled equations that describe DFWM in Kerr-like media. In the second section, I present a model of DFWM in the limit where the probe intensities are much weaker than the pump intensities, and discuss experimental results in intrinsic germanium. The third section reviews theoretical treatments of the full four-wave interaction, discusses a numerical technique for the solution of the four-wave equations, and compares experimental results to this numerical mociel. A discussion and summary is given in the fourth section.

A. The third-order nonlinear susceptibility and DFWM The third-order susceptibility as discussed in the introduction is a well defined constant for a given medium. In resonant media, Eq, 1 is generally valid only for intensities weak compared to some "saturation" 
intensity, and the higher order terms dominate at intensities near the saturation intensity. There has been a tendency in recent months to identify the nonlinearity in any wave mixing process that looks like DFWM with a third-order nonlinearity. ${ }^{3}$ Usually this identification results in an intensity dependent $x_{3}$. Since I feel this runs counter to the philosophy of the perturbation expansion given in Eq. 1, I will avoid such identifications throughout this thesis. The confusion results because one can either count the number of distinguishable waves in an interaction, and label the interaction accordingly, or one can count the number of waves used in the theoretical expressions for the interaction. I should also note that in the presence of a resonance and significant absorption, $x_{3}$ is complex.

With this much out of the way, I can now turn to a more precise definition of $x_{3}$ and a discussion of its properties.

Each term in Eq. 1 can be considered as an expression for the nth order polarizability. The nth rank susceptibility is the $(n+1)$ th-rank tensor that relates the polarizability (at a given frequency) to the electric fields (possibly at different frequencies). In discussing this nonlinear polarizability, I employ the convention of Maker and Terhune, ${ }^{4}$ who wrote the third-order 
polarizability as

(2) $P_{i}(3)\left(v_{4}\right)=D x_{3}^{i j k 1}\left(v_{4} ; v_{1}, v_{2}, v_{3}\right) E_{j}\left(v_{1}\right) E_{k}\left(v_{2}\right) E_{1}\left(v_{j}\right)$

Conservation of energy requires $v_{4}=v_{1}+v_{2}+v_{3}$.

The factor $D$ is called the degeneracy factor and counts possible permutations of the three frequencies. The indices $i, j, k$ and $l$ refer to cartesian coordinates, and for crystalline structures the coordinate system is defined by the crystal axes.

In Maker and Terhune's notation, the electric field is given by $E_{j}(r, t)=\operatorname{Re}\left[E_{j}(v) e^{-i v t}\right]$. Note that $E_{j}(v)$ is twice the actual Fourier component of the electric field. Another common convention is to use the true Fourier component, in which case the third-order susceptibility must be four times the magnitude of that used here.

For the sake of simplicity, I consider only media with inversion symmetry (either cubic crystals or isotropic media). The inversion symmetry, and the symmetry of rotations by $90^{\circ}$ result in a great reduction of the number of independent components of $x_{3}^{j j k 1} .^{2}$ The independent components are denoted $x_{3}^{1111}, x_{3}^{1122}, x_{3}^{1212}$, and $x_{3}^{1221}$, where 1 and 2 are any of the three axes, $x, y$ or $z$. All other components are zero. In isotropic media, the further restriction $x_{3}^{1111}=x_{3}^{1122}+x_{3}^{1212}+x_{3}^{1221}$ applies. 
In the case of DFWM, the frequencies are related by $v_{1}=v_{2}=-v_{3}$. Under this condition, permutation of the middle frequency terms gives $x_{3}^{1122}(v ; v, v,-v)=x_{3}^{1212}(v ; v, v,-v)$.

If the fields are all polarized along a single direction, it is often convenient to use an effective $x_{3}$. Table 1 relates the effective $x_{3}$ to $x_{3}^{i j k 1}$ for various polarizations. For arbitrary orientation of the input field relative to the crystal axes, the polarizability of the medium may not be along the same orientation as the driving field.

I now turn to the derivation of the coupled equations describing DFWM in a Kerr-like medium. ${ }^{5}$ To describe the nonlinear interaction of the four waves in the medium, four coupled equations are used. These equations will be derived by obtaining the polarizability, inserting it into the wave equation, and "phase matching," that is matching terms in the wave equation that have similar spatial wave vectors. The phase-matching splits the wave equation into folir equations each of which describes the propagation of a single wave. 
Table 1

Effective $x_{3}$ as a function of electric field orientation.

Material E-Field Polarization Effective $X_{3}$

I sotropic

Cubic

Cub ic

Cub ic
[111] Crystal Axis $x_{3}^{1111}=x_{3}^{1122}+x_{3}^{1212}+x_{3}^{1221}$

$x_{3}^{1} 111$

$\left(x_{3}^{1111}+x_{3}^{1122}+x_{3}^{1212}+x_{3}^{1221}\right) / 2$

$\left[x_{3}^{11 i 1}+2\left(x_{3}^{1122}+x_{3}^{1212}+x_{3}^{1221}\right)\right] / 2$ 
The word "degenerate" in DFWM refers to the characteristic that all four waves in the interaction have the same frequency. Hence the third-order polarizability is given in terms of the total field (including all four waves) by

(3) $P_{i}(3)(\nu)=3 x_{3}^{i j k 1}(\nu ; \nu, v,-v) E_{j}(\nu) E_{k}(\nu) E_{1}(-\nu)$

The degeneracy factor of three applies because there are three positions where the $-\nu$ term could appear. For the sake of simplicity, I assume that all the waves are polarized along the same direction, so that the indices may be dropped and an effective $x_{3}$ used. Implicit in this assumption for cubic media is the additional constraint that the fields are polarized along a symmetry axis in the crystal, so that the polarization lies along the same axes and Table 1 applies. With this assumption, I have

$$
p^{(3)}(v)=3 x_{3} E^{2}(v) E *(v)
$$

since for $E(r, t)$ to be real, $E(-\nu)=E^{*}(\nu)$.

The total electric field is the sum of the four interacting waves,

$$
\begin{aligned}
E(v) & =E_{f} e^{i\left(k_{z} z+k_{x} x\right)}+E e^{-i\left(k_{z} z+k_{x} x\right)} \\
& +E_{1} e^{i\left(k_{z} z-k_{x} x\right)}+E_{2} e^{-i\left(k_{z} z-k_{x} x\right)} .
\end{aligned}
$$




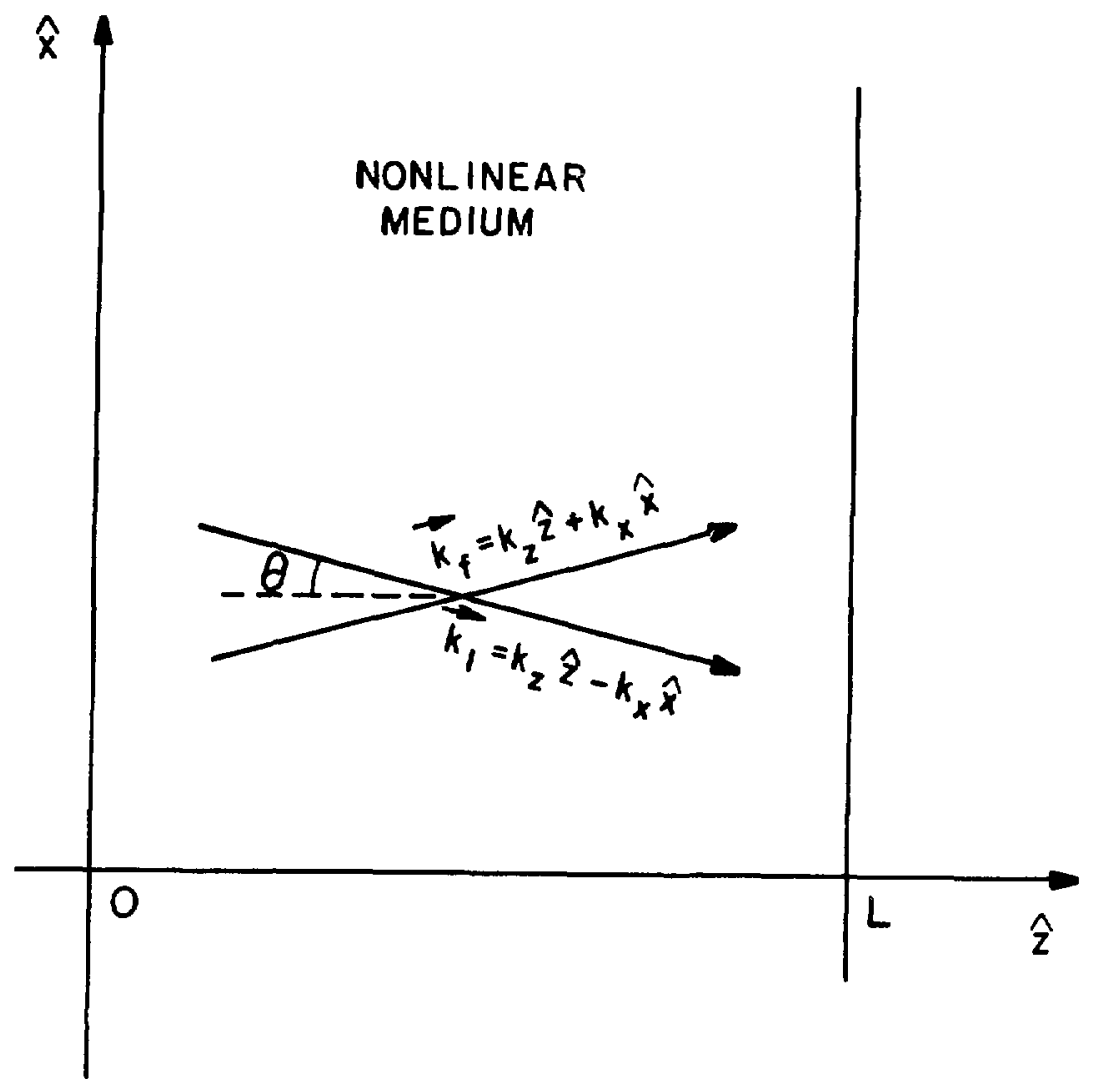

Figure III-1. Geometry for analys is of DFWM. Only plane wave analysis is done in the thesis. Thus the symmetry is defined by the boundaries of the medium which are taken as parallel to the $x-y p l a n e$. The wave vectors are in the $x-z$ plane. This combination insures that only variations in $z$ occur in the wave equation. That is $d / d x$ and $d / d y$ $\rightarrow 0$. 
Here, I have assumed that the polarization lies along the $y$ axis and the $z$ axis is symmetric with respect to the propagation of the four waves (see Fig. 1). The boundaries of the medium are parallel to the $x-y$ plane. It is obvious from Eq. 5 that the triple product of the fields needed in Eq. 3 is a mess.

To avoid this mess, I introduce phase-matching. The concept of phase-matching is that in order for a wave to exchange energy in an interaction, the relative phase of the wave and a component of the polarizability must be approximately the same over macroscopic distances. If this is not the case, destructive interference prohibits the exchange of energy. Thus, phase matching ideally requires the same relative phase for the wave and polarizability over the full interaction length, or the interaction will be reduced. Although each of the fields $E_{\alpha}$ is complex, and has a phase that will vary slowly in space, the wave vector gives the dominant contribution to the total phase. Phase-matching is thus achieved by determining which terms in the triple product $E^{2} E *$ have the same wave vector as some wave in the interaction.

For instance, the terms which are phase-matched to the forward pump beam $E_{f}$ are found by noting that any term of the form $\left|E_{\alpha}\right|^{2} E_{f} \exp \left[i\left(k_{z} z+k_{x} x\right)\right]$ is phase-matched, as is the term $E_{1} E_{2} E_{b}^{*} \exp \left[i\left(k_{z} z+k_{x} x\right)\right]$ (which occirs twice). 
There are many terms such as $E_{2}^{2} E_{f}^{*} \exp \left[-i\left(3 k_{z} z-k_{x} x\right)\right.$ or $E_{1} E_{2}^{*} E_{b} \exp \left[i\left(k_{z} z-3 k_{x} x\right)\right]$ which do not phase match to any of the above four waves. It is not the case that these terms phase-match to some other wave not yet accounted for, because the magnitude of the wave vector is never right, that is $\left|3 k_{z} z-k_{x} x\right|=k=\sqrt{\left(k_{z}^{2}+k_{x}^{2}\right)}$. Hence, these terms cannot be phase-matched to any wave at the frequency $v$.

Phase matching is essentially the requirement that momentum be conserved. That is, just as energy conservation requires $v=v_{1}+v_{2}+v_{3}$, conservation of momentum approximately requires $k=k_{1}+k_{2}+k_{3}$. The approximation applies because the total momentum of a proton is given by $\hbar k^{\prime}$ where $k^{\prime}=k+\Delta k=[n+\Delta n(I)] \nu / c$. Here, $n$ is the linear index of refraction (resulting from $\left.x_{l}\right), \Delta n(I)$ is the nonlinear, intensity-dependent index (resulting from $x_{3}$ ), and $\Delta n \ll n$.

Finally, I note that the common assertion ${ }^{6}$ that there is no phase-matching constraint on the four-wave mixing geometry presented here is incorrect. There is no constraint on the angle $\theta$ between the probe and the pump beams, but for perfect phase-matching, the pump beams must be exactly counterpropagating. To the degree that this is not the case, a phase-mismatch occurs. 
Summarizing the results so far, the third-order nonlinear polarizability can be written as the sum of five parts,

(6)

$$
p^{(3)}=P_{f} e^{i\left(k_{z} z+k_{x} x\right)}+P_{b} e^{-i\left(k_{z} z+k_{x} x\right)}
$$

$$
+P_{1} e^{i\left(k_{z} z-k_{x} x\right)}+P_{2} e^{-i(k z-k x)}+P_{n p m}
$$

where $P_{n p m}$ refers to the part of the polarizability that is not phase-matched, and, for example,

$$
P_{f}=3 x_{3}\left\{\left(\left|E_{f}\right|^{2}+2\left|E_{b}\right|^{2}+2\left|E_{q}\right|^{2}+2\left|E_{2}\right|^{2}\right) E_{f}+2 E_{1} E_{2} E_{b} *\right\}
$$

The wave equation third order in the nonlinear polarizability is

$$
\nabla^{2} E(v)+\frac{n^{2} v^{2}}{c^{2}} E(v)=\frac{-4 \pi v^{2}}{c^{2}} P(3)(v)
$$

Here the first order polarizability is incorporated into the index of refraction $n$.

The wave equation is customarily treated in the slowly varying envelope approximation (SVEA). This approximation amounts to assuming that the variations in the amplitudes E occur only on a scale that is large compared to the wavelength. So putting $\vec{\xi}=x \hat{x}+z \hat{z} I$ have, for example,

$$
\left|\frac{d^{2} E_{f}}{d \zeta^{2}}\right| \ll k\left|\frac{d E_{f}}{d \zeta}\right|
$$

and 
(10) $\quad \nabla^{2}\left(E_{f} e^{i k \zeta}\right) \approx\left(2 i k \frac{\partial E_{f}}{\partial \zeta}-k^{2} E_{f}\right) e^{i k \zeta}$

Since $k^{2}=n^{2} v^{2} / c^{2}$, the second term in Eq. 8

cancels. I now have four terms on the left hand side of

Eq. 8, one for each field, and five terms in the

polarization on the right hand side, four of which

phase-match (i.e., have identical exponentials) with the

terms on the left hand side. Equating coefficients of the

exponentials yields the four coupled differential equations

(11a) $\frac{d E_{f}}{d z}=i \beta\left\{\left[\left|E_{f}\right|^{2}+2\left|E_{b}\right|^{2}+2\left|E_{1}\right|^{2}+2\left|E_{2}\right|^{2}\right] E_{f}+2 E_{1} E_{2} E_{b} *\right\}$,
(11b) $\frac{d E_{b}}{d z}=-i \beta\left\{\left[\left|E_{b}\right|^{2}+2\left|E_{f}\right|^{2}+2\left|E_{1}\right|^{2}+2\left|E_{2}\right|^{2}\right] E_{b}+2 E_{1} E_{2} E_{f} *\right\}$,
(11C) $\frac{d E_{1}}{d z}=i \beta\left\{\left[\left|E_{1}\right|^{2}+2\left|E_{2}\right|^{2}+2\left|E_{f}\right|^{2}+2\left|E_{b}\right|^{2}\right] E_{1}+2 E_{f} E_{b} E_{2} *\right\}$,

(11d) $\frac{d E_{2}}{d z}=-i \beta\left\{\left[\left|E_{2}\right|^{2}+2\left|E_{1}\right|^{2}+2\left|E_{f}\right|^{2}+2\left|E_{b}\right|^{2}\right] E_{2}+2 E_{f} E_{b} E_{1} *\right\}$,

where $\beta=6 \pi v x_{3} /(n c \cos \theta)$. In obtaining Eqs.

(11), I have employed the symmetry of Fig. 1 to transform

the equations by noting that the steepest gradient for all quantities occurs along the $z$ axis. Thus $d / d x=0$, and a transformation of variables simply introduces the $\cos (\theta)$ dependence. Note that the condition $\theta=\pi / 2$ or $\cos (\theta)=0$ is singular, because at that point the beams are propagating along the $\mathrm{x}$ axis and remain in the medium forever. This condition is unphysical. 
The four Eqs. 11 form the basis for the theories presented in this chapter. The term in the square brackets in each of these equations produces a change in index of refraction for the wave it multiplies. Since each of these square brackets is different, the nonlinear index experienced by each wave is generally different. The last term in each equation is the coupling term.

Various solutions to these coupled equations have been presented. My purpose in the remainder of this chapter is to present the solutions and compare them to experiment when possible. The features present in these solutions are important in understanding the more complex wave mixing processes discussed in later chapters.

B. DFWM in the small signal limit

Three variations of the small-signal theory have been published. The original papers are by Hellwarth, ${ }^{6}$ Yariv and Pepper, ${ }^{7}$ and Marburger and Lam. ${ }^{8}$ The authors differ somewhat in their approaches, and consequently in their results. In the simplest form, their results are a 11 identical, though some are more general than others. The treatment given here most closely parallels that given by Marburger and Lam, which is the most general.

The small signal limit means that the probe fields, $E_{1}$ and $E_{2}$, are much smaller than the pump fields, $E_{f}$ 
and $E_{b}$. The four coupled Eqs. 11 can then be further simplified by dropping the second order terms in $E_{1}$ and $E_{2}$. This gives

(12a) $\frac{d E_{f}}{d z}=i \beta\left[\left|E_{f}\right|^{2}+2\left|E_{b}\right|^{2}\right] E_{f}$
(12b) $\frac{d E_{b}}{d z}=-i \beta\left[\left|E_{b}\right|^{2}+2\left|E_{f}\right|^{2}\right] E_{b}$
(12c) $\frac{d E_{1}}{d z}=2 i \beta\left\{\left[\left|E_{f}\right|^{2}+\left|E_{b}\right|^{2}\right] E_{f}+E_{f} E_{b} E_{2} *\right\}$

and

(12d) $\frac{d E_{2}}{d z}=-2 i \beta \quad\left\{\left[\left|E_{r}\right|^{2}+\left|E_{b}\right|^{2}\right] E_{2}+E_{f} E_{b} E_{1} *\right\}$

The pump field Eqs. $12 \mathrm{a}$ and $b$ are decoupled from the probe fields.

The solution for the pump fields is trivial.

Substituting $E_{\alpha}=\rho_{\alpha} \exp \left(i \phi_{\alpha}\right)$, and separating

real and imaginary parts gives

$$
\frac{d \rho_{f}}{d z}=\frac{d \rho_{b}}{d z}=0
$$

and

(14a) $\quad \frac{d \phi_{f}}{d z}=+\beta\left[\rho_{f}^{2}+2 \rho_{b}^{2}\right]$

(14b) $\quad \frac{d \phi_{b}}{d z}=-\beta\left[\rho_{b}^{2}+2 \rho_{f}^{2}\right]$

Integration gives $\rho_{f}$ and $\rho_{b}$ constant. In the probe field Ers. $12 c$ and $d$ only the sum $\phi_{+}=\phi_{b}+$ $\phi_{f}$ occurs and 


$$
\phi_{+}=\beta\left[\rho_{b}^{2}-\rho_{f}^{2}\right] z
$$

To solve the equations for the probe waves, I note that the nonlinear index terms in $\mathrm{Eq} .12 \mathrm{c}$ and $\mathrm{d}$ are identical except for the sign. Transforming by

$$
E_{1}=A_{1} \exp [ \pm i \Delta n z]
$$

where $\Delta n=2 B\left(\rho_{f}^{2}+\rho_{b}^{2}\right)$, I obtain

(17a) $\frac{d A_{1}}{d z}=i \kappa A_{2}^{*} e^{i \phi_{+}}$

and

(17b) $\quad \frac{d A_{2}}{d z}=-i k A_{1}^{*} e^{i \phi_{+}}$

where I define $k=12 \pi \nu x_{3} \rho_{f} \rho_{b} / n c \cos \theta$.

The solution to these equations is obtained by substituting from one into the other and solving the resulting second order differential equation. Application of the boundary conditions $A_{1}(z=0)=0$ and $A_{2}(z=L) \neq 0$ results in the solution

$$
A_{1}(z)=\frac{-i K A_{2}^{*}(L) e^{-i \phi_{+}} \sin w z}{w \cos w L+\frac{i}{2} \frac{d \phi_{+}}{d z} \sin w L}
$$

where

$$
w=\sqrt{\kappa^{2}+\frac{1}{4}\left(\frac{d \phi_{+}}{d z}\right)^{2}}=\kappa \sqrt{1+\left(\rho_{b}^{2}-\rho_{f}{ }^{2}\right)^{2} / 16 \rho_{f}{ }^{2} \rho_{b}{ }^{2}}
$$


This is the result that has made DFWM almost synonymous with phase conjugation. The wave amplitude of the generated wave $A_{1}$ is proportional to the complex conjugate of the input wave $A_{2}$, and $A_{1}$ propagates in exactly the opposite direction to $A_{2}$ (actually this is subject to the constraint that the pump beams are exactly counterpropagating). The reflectivity is defined as the ratio of the generated wave intensity to the input signal intensity:

$$
R=\left|\frac{A_{1}(L)}{A_{2}(L)}\right|^{2}=\frac{\sin ^{2}(w L)}{\cos ^{2}(w L)+\left(\rho_{b}{ }^{2}-\rho_{f}\right)^{2} / 16 \rho_{f}^{2} \rho_{b}{ }^{2}} .
$$

This form of the reflectivity was originally given by Marburger and Lam. ${ }^{8}$ For constant $\rho_{f} \rho_{b}$, that is for constant coupling coefficient $k$, the reflectivity is maximum when $\rho_{f}=\rho_{b}$. This is because when these two beams are not equal in intensity, the nonlinear index experienced by each wave is different. This difference causes the phase $\phi_{+}$to vary and creates a phase-mismatch in the coupling terms of the probe wave equations, which results in a decreased efficiency for the wave mixing process.

The reflectivity for the case $\rho_{f}=\rho_{b}$ is given by

$$
R=\tan ^{2} \kappa L
$$


This is the expression originaily given by Yariv and Pepper. ${ }^{7}$ Hellwarth's solution ${ }^{6}$ reduces this even further by the assumption that $k L \ll 1$, so that $R=$ $(k L)^{2}$.

In Eq. 20, when $K L=\pi / 2, R+\infty$. This results from ignoring the depletion of the pump beam intensity by the interaction. This condition has been dubbed the oscillation condition, because the "reflected" wave can be nonzero for zero input probe wave intensity. A laser oscillator has been built based on this observation usirg barium titanate, which has a large non-kerr-like non 1 inearity. ${ }^{9}$

Experimental results in intrinsic germanium

DFWM experiments have been performed in several samples of intrinsic Ge. A general description of the DFWM experiment used to obtain these results is given in Chapter II. Germanium has a large third-order nonlinear susceptibility in the infrared due to nonlinear response of bound electrons. The samples employed in these experiments have varying lengths and effective nonlinear susceptibilities (due to variations in crystal orientation). The results of these measurements are plotted in Fig. 2 . 


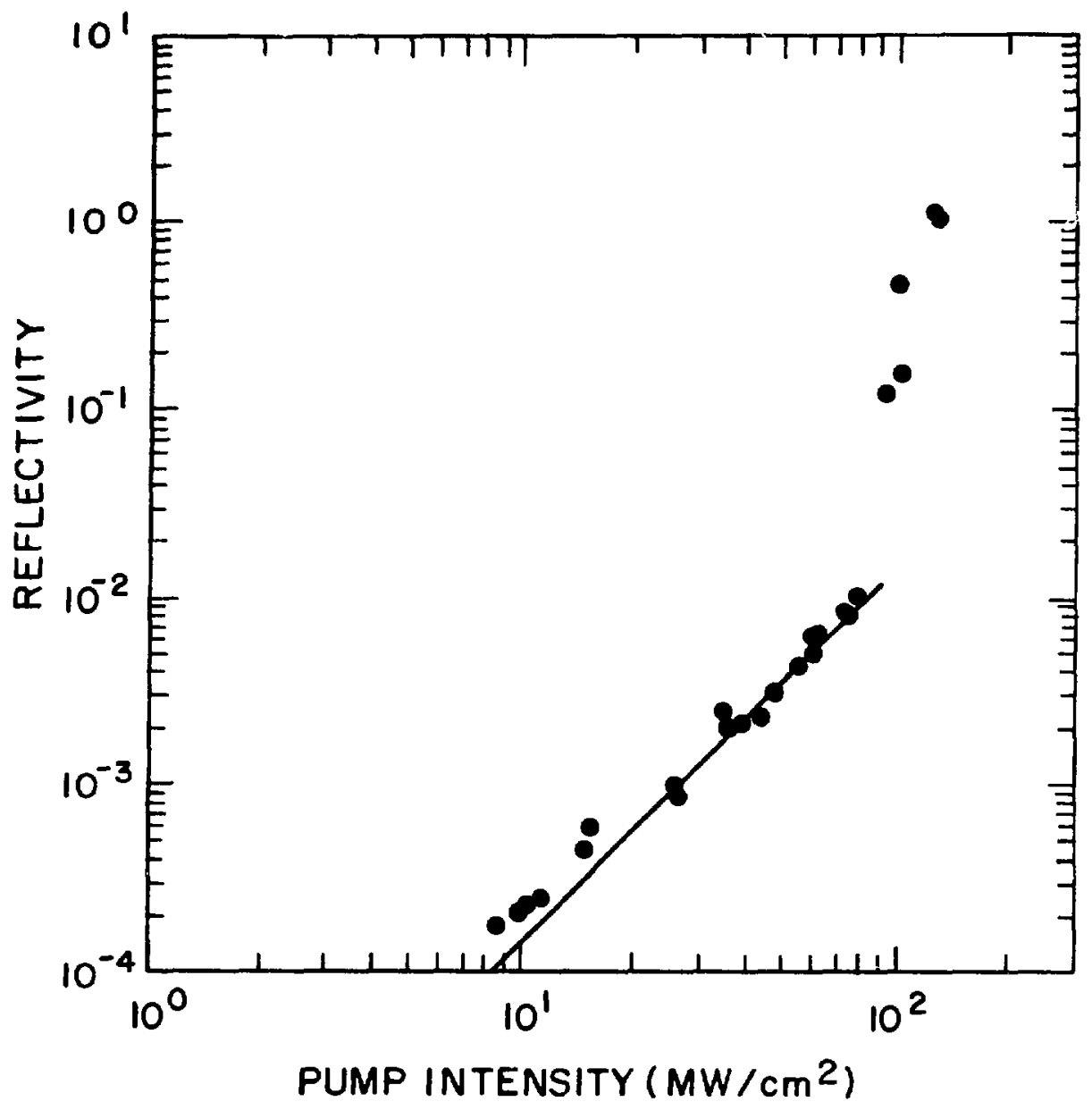

Figure III-2a. DFWM in a 3-mm sample of optical grade, multicrystalline Ge. The curve is a fit of Eq. 20 to the data using $X_{3}=3.2 \times 10^{-11}$ esu. Plasma formation causes enhanced reflectivity above $80 \mathrm{MW} / \mathrm{cm}^{2}$. All of the data presented in Figs. 2 are taken at $10.6 \mu \mathrm{m}$. 


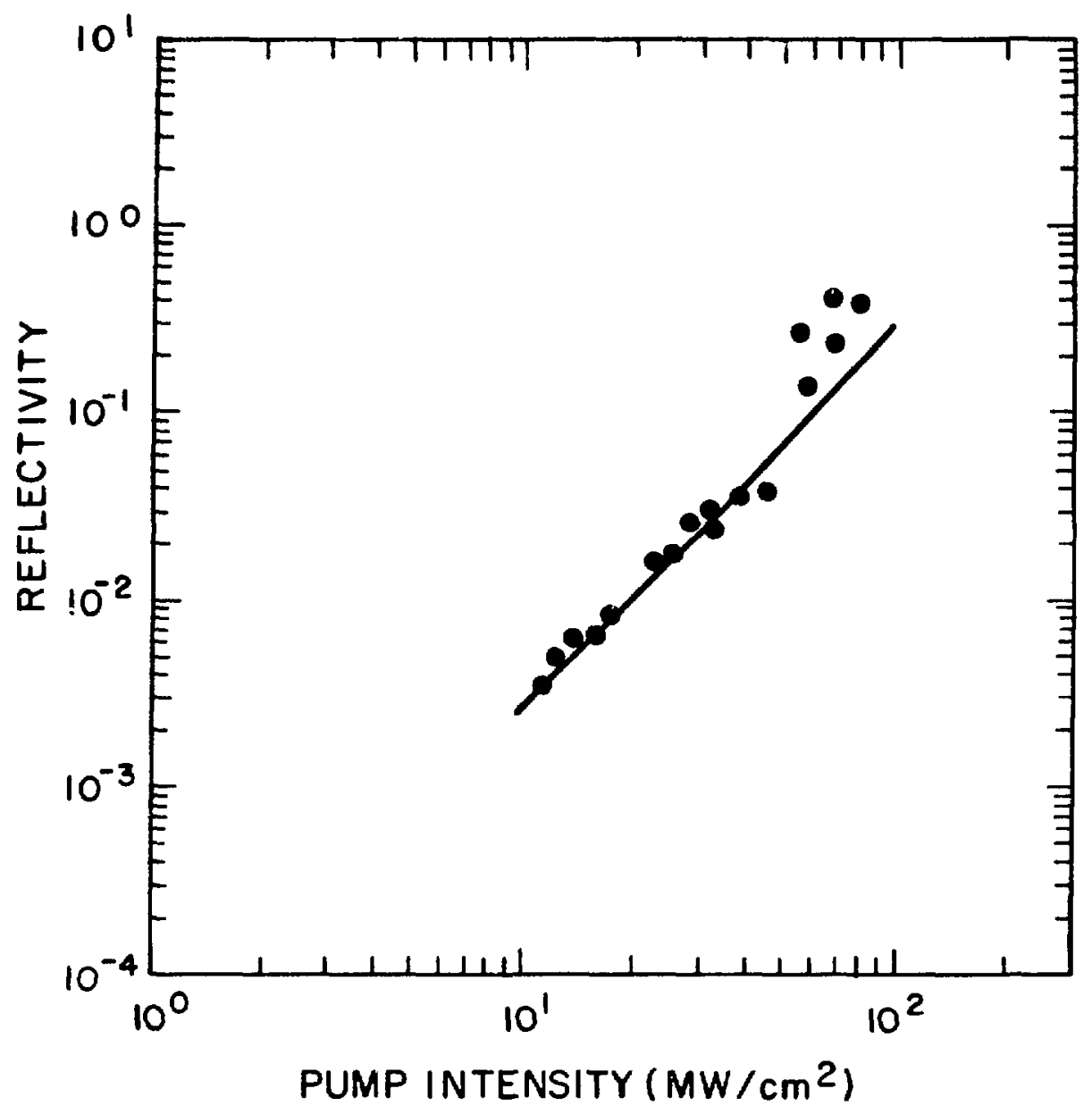

Figure III-2b. DFWM in a 1-cm sample of intrinsic, multicrystalline Ge. The curve is a fit of Eq. 20 to the data using $x_{3}=4.4 \times 10^{-11}$ esu. Plasma formation occurs at about $70 \mathrm{MW} / \mathrm{cm}^{2}$. 


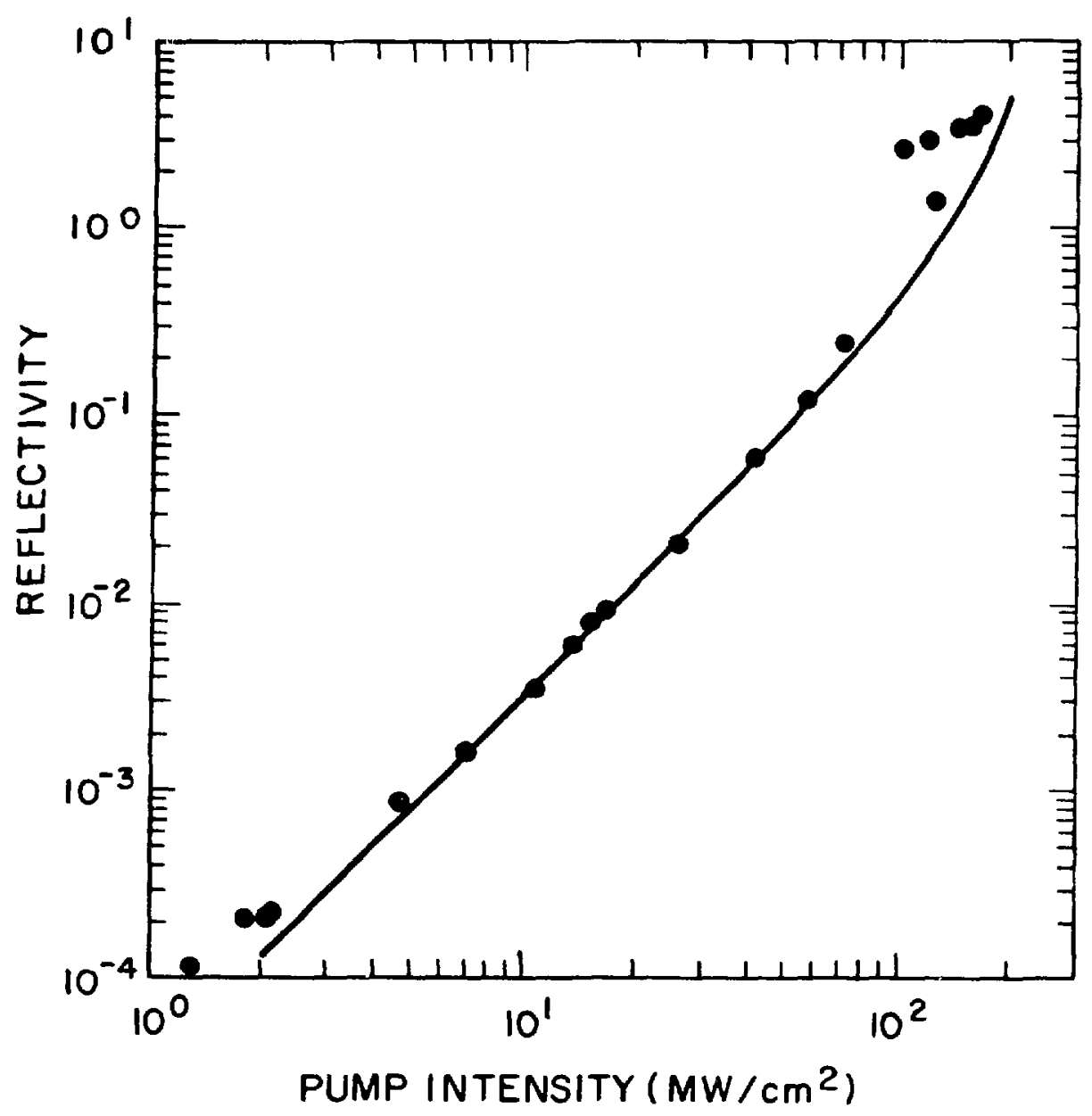

Figure III-2c. DFWM in a 2-cm sample of optical grade, single-crystalline Ge. The polarization is along the [100]-crystal axis. The curve is a fit of Eq. 20 to the data using $x_{3}=2.5 \times 10^{-1}$ esu. The values of $x_{3}$ in $F i g s . a, b$, and $c$ are in reasonable agreement with the values in Appendix B. Plasma formation occurs above 80 $\mathrm{MW} / \mathrm{Cm}^{2}$ and slight enhancement is observed. 


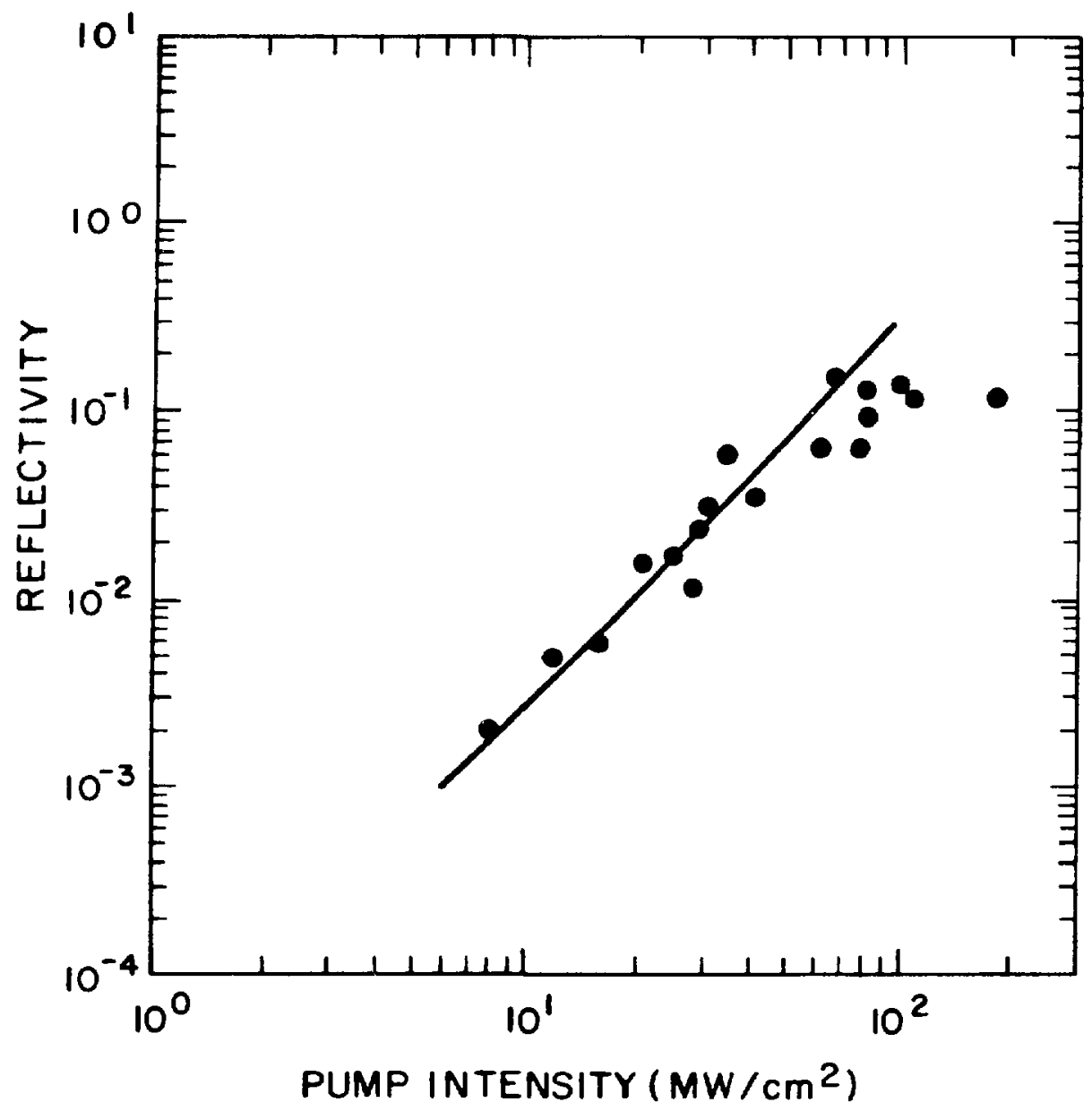

Figure III-2d. DFWM in a 4-cm sample of intrinsic, single-crystalline Ge. The polarization is along the [100]-crystal axis. The curve is a fit of Eq. 20 to the data by $x_{3}=7.1 \times 10^{-11}$ esu, less than $7 / 3$ the expected value (see Table 1 and Appendix B). This is because the sample is long compared to the laser pulse. Plasma formation above $90 \mathrm{MW} / \mathrm{cm}^{2}$ causes a degradation in reflectivity in this long sample. (For a discussion, see Chapter VI.) 
The duration of the laser pulse is nominally 1.6 ns. The application of a time independent theory to these experiments requires that the response time of the nonlinear medium be much shorter than the laser pulse, and that the optical path length can be traversed in a time much shorter than the laser pulse duration. In Ge, the nonlinearity is due to bound electrons, which respond on subpicosecond time scales. The optical path length in a dielectric is $n L$, where $n$ is the index of refraction. Thus I must have $\mathrm{nL} / \mathrm{c} \ll 1.6 \mathrm{~ns}$, or $\mathrm{L} \ll 12 \mathrm{~cm}$. The samples shown in the figure range in length from $0.3 \mathrm{~cm}$ to $4 \mathrm{~cm}$. The only sample that shows a substantial deviation from a realistic value for the effective $x_{3}$ is the 4-cm-long sample, for which the time independent model is inapplicable. ${ }^{10}$ An $I^{2}$ dependent line still fits the data below the plasma formation threshold. The other samples are all modeled well by the relation $R=\tan ^{2} \mathrm{KL}$ for equal pump intensities, although in fact, the only sample that shows any divergence from the simpler $R=(K L)^{2}$ model is the $2-c m-1$ ong sample. The divergence that occurs at high intensities is due to plasma formation and is dealt with in Chapter VI.

Values for the third-order nonlinear susceptibility of Ge have been published by J. J. Wynne. 11 However, more recent determinations of these same coefficients by myself 
have yielded significantly smaller values. 12 A reprint of my work in determining these coefficients is given in Appendix B. The values for the effective $x_{3}$ 's used above are in good agreement with the values given in Appendix B.

C. DFWM in the large signal $1 \mathrm{imit}$

In the previous section, I presented a solution for the coupled Eqs. 11 in the limit of small probe intensity, or negligible pump depletion by the four-wave mixing. In this section, I will discuss solutions of the full four-wave interaction, where only the SVEA that lead to Eqs. 11 is used.

The analytic solution of the four coupled Eqs. 11 is beyond the scope of this thesis. My purpose here is to briefly discuss the various attempts at solutions that others have made, to present a form of the equations suitable for numerical integration, and to compare experimental results to the numerical model.

The first attempt at a solution to Eq. 11 was made by Hsu. 13 In his treatment, Hsu ignores the nonlinear index terms in these equations, in an attempt to isolate the effect of pump depletion. However, as we have seen in the smali-signal theory presented above, these terms are important whenever the pump intensities are not equal 
throughout the interaction volumn. If pump depletion is present, the counterpropagating pump beams cannot be equal at all points along the interaction. Thus deletion of the nonlinear index terms introduces significant error into Hsu's solution.

The second attempt at a solution of Eqs. 11 was reported by Marburger and Lam. 14 These authors are obviously aware of the importance of including the nonlinear index terms, since they also worked out the small-signal theory given above. Marburger and Lam employ a collinear geometry, and attempt to distinguish between pump and probe waves by polarization. The problem with their solution is that, in a collinear geometry, the terms in the polarizability such as $x_{3}^{1212} E_{f} E_{2} E_{b}^{*}$ are phase matched. This term couples a wave to the phase conjugate of the backward pump beam with the forward pump beam and the input probe beam acting as "pump beams."

This wave interferes with the phase conjugate wave produced by the desired interaction arising from $x_{3}^{1221}$ $E_{f} E_{b} E_{2}^{*}$. Since Marburger and Lam did not include these terms in their analysis, their solution is incorrect; it is also clear that a solution of the complete equations for this case would not yield a simple phase conjugate relation between the input signal and the generated wave anyway. 
An analytic solution to Eqs. 11 has been provided by Winful, 15 and independently by Rigrod. ${ }^{16}$ These solutions basically agree, and I believe them to be correct. Although a thorough treatment of this solution is beyond the scope of this thesis, I review the general idea here. Three conserved quantities may be obtained from Eqs. 11, which follow from the way in which energy is transferred from one wave to another in a lossless Kerr-like medium. These three conserved quantities, plus the boundary conditions on the input intensities, allow the four coupled equations to be reduced to a single equation. This equation relates a generalized coupling coefficient to an elliptic integral of the first kind, which depends on the conserved quantities and the boundary conditions. The solution of this relationship gives the transmitted signal intensity, from which the reflected intensity can be obtained. Although this solution gives many interesting results, to compare it with my data would require an iteration process, since the coupling coefficient cannot be defined a priori in the elliptic integral relation. I present a form of the equations suitable for numerical integration from which I have obtained a model for the decrease in reflectivity as a function of increasing probe intensity (and hence pump depletion). This model can be compared directly to 
experimental results.

The transformation to a form convenient for numerical integration is simple. I substitute

(21) $\quad E_{j}=\rho_{j} \exp \left(2 i \beta \rho^{2} z+i \phi_{j}\right)$

where $\rho^{2}=\rho_{f}^{2}+\rho_{b}^{2}+\rho_{1}^{2}+\rho_{2}^{2}$ into Eq. 11.

Then, defining the total phase as $\phi=\phi_{f}+\phi_{b}-\phi_{1}-$

$\phi_{2}$, I obtain

(22a) $\frac{d \rho_{f}^{2}}{d z}=\frac{-d \rho_{b}^{2}}{d z}=-4 \beta \rho_{f} \rho_{b} \rho_{1} \rho_{2} \sin \phi$

(22b) $\quad \frac{d \rho_{1}^{2}}{d z}=\frac{-d \rho_{2}^{2}}{d z}=4 \beta \rho_{f} \rho_{b} \rho_{1} \rho_{2} \sin \phi$

and

$\begin{aligned} \text { (22c) } & \frac{d \phi}{d z}=\beta\left(\rho_{f}^{2}-\rho_{b}{ }^{2}+\rho_{1}{ }^{2}-\rho_{2}{ }^{2}\right) \\ + & 2 B\left\{\frac{\rho_{1} \rho_{2} \rho_{f}}{\rho_{b}}-\frac{\rho_{1} \rho_{2} \rho_{b}}{\rho_{f}}+\frac{\rho_{f} \rho_{b} \rho_{2}}{\rho_{1}}-\frac{\rho_{f} \rho_{b} \rho_{1}}{\rho_{2}}\right\} \cos \phi .\end{aligned}$

The three conservation laws mentioned above are obtained from the equations for the amplitudes $\rho_{j}$. These are

(23a) $\rho_{f}^{2}+\rho_{f}^{2}=$ constant,

(23b) $\rho_{1}^{2}-\rho_{b}^{2}=$ constant,

and

(23c) $\rho_{1}^{2}+\rho_{2}^{2}=$ constant.

59 
The conservation laws of Eqs. 23 can be understood as follows: since the medium is lossless, the sum of all the intensities (Eq. a - Eq. b + Eq. c) must be a constant. Conservation of momentum requires that for each unit of energy scattered from the forward pump beam, an equal amount must be scattered from the backward pump beam (Eq. a - Eq. b), and similarly for the probe beam (Eq. C). The conservation laws of Eqs. 23 result from a linear combination of these relations.

Returning to Eqs. 22, if I take $\rho_{2}$ as the input signal and $\rho_{1}$ as the generated wave, so that $\rho_{1}(z=0)=0$, there appears to be a singularity in the equation for $\phi$. In fact, the boundary conditions require $\phi(z=0)=\pi / 2$, so that no singularity occurs. The apparent singularity is the result of dividing by $\rho_{1}$ before application of the boundary conditions.

A numerical solution for the four coupled waves can be obtained from the differential equations for $\rho_{1}$ and $\phi$ using the conservation laws of Eq. 23. Since the boundary conditions that determine the constants of Eqs. 23 are not al1 known at either the $z=0$ or $z=L$ boundaries, an iterative process must be used to obtain this solution. In the iterative process I have developed, the values for the various parameters are "guessed" at the $z=0$ boundary, and a solution is generated based on this guess. The solutions at 
the boundaries are compared to the boundary conditions, and the process is repeated until there is good agreement between the numerical solution and the input boundary conditions.

The results of this numerical calculation are presented in Fig. 3a. Here the ratio of the calculated reflectivity to the reflectivity in the small-signal limit is plotted as a function of the relative signal intensity, for several values of the small-signal reflectivity (or coupling coefficient $\beta$ ). The common factor in these curves is that the reflectivity begins to decrease whenever the reflected intensity is as large as $1 \%$ of the pump intensity.

Experimental results in the pump depletion regime are shown in Fig. 3b. The sample is a 2-cm-long piece of intrinsic Ge with the laser poiarization oriented along the [111]-crystal axis for maximum effective $x_{3}$. The points are the experimental data, and the curve is the numerical model presented above. Although the scatter in this plot is fairly large, the mean absolute deviation of the data from the model is only $8.2 \%$. The scatter is due to the large intensity dependence of the reflectivity (approximately $I_{\text {pump }}^{2}$ ), and the unavoidable fluctuations in laser intensity. Although some data with very large fluctuations could be discarded, the remaining data still have 


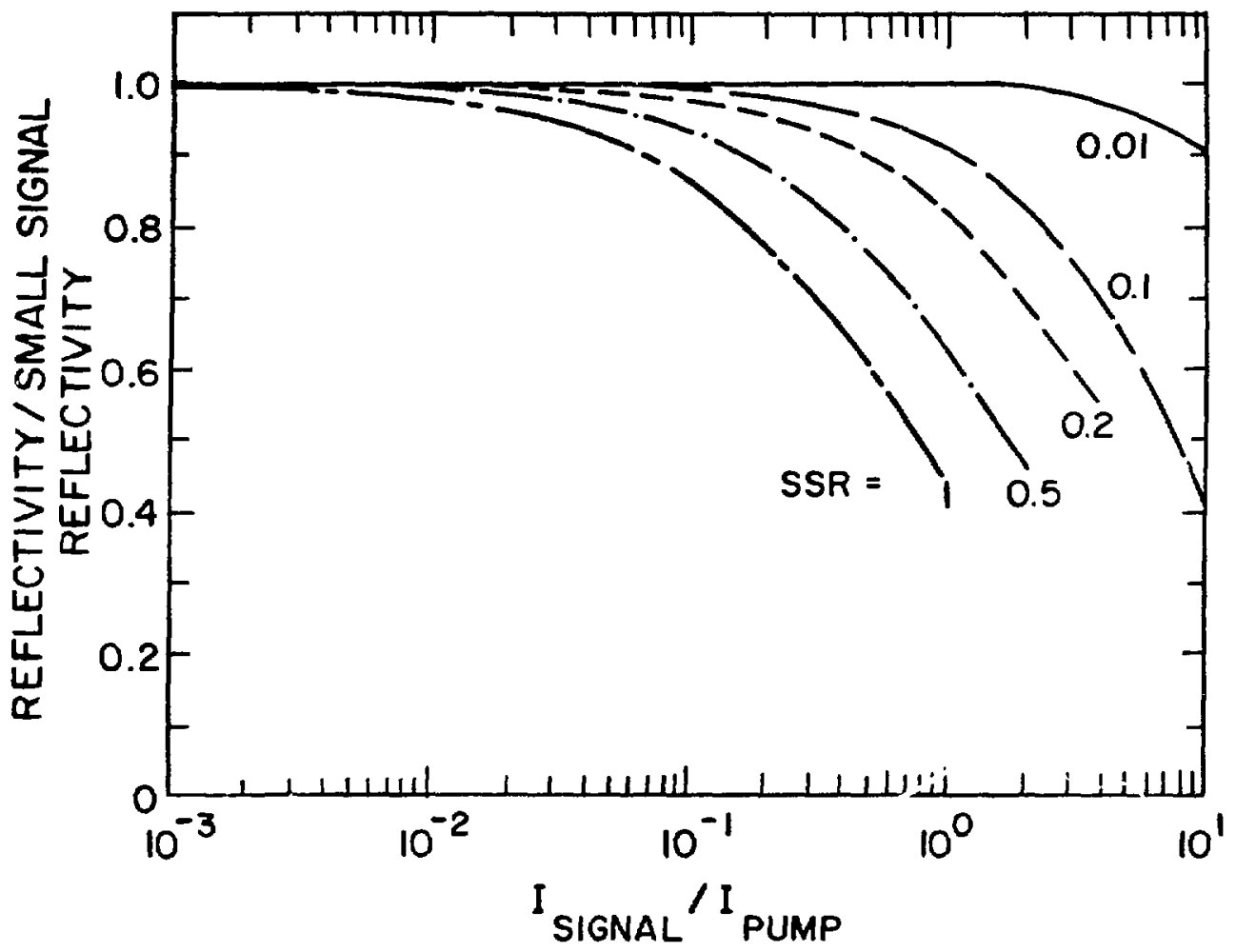

Figure III-3a. Theoretical reflectivity in the regime of significant pump depletion. The curves show the reflectivity normalized to the small-signal reflectivity (SSR) as a function of relative probe intensity for several values of the SSR. The common feature of these curves is that the reflectivity begins to decline whenever

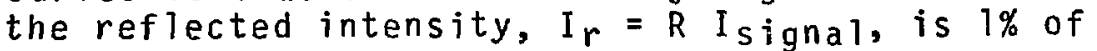
I pump. 


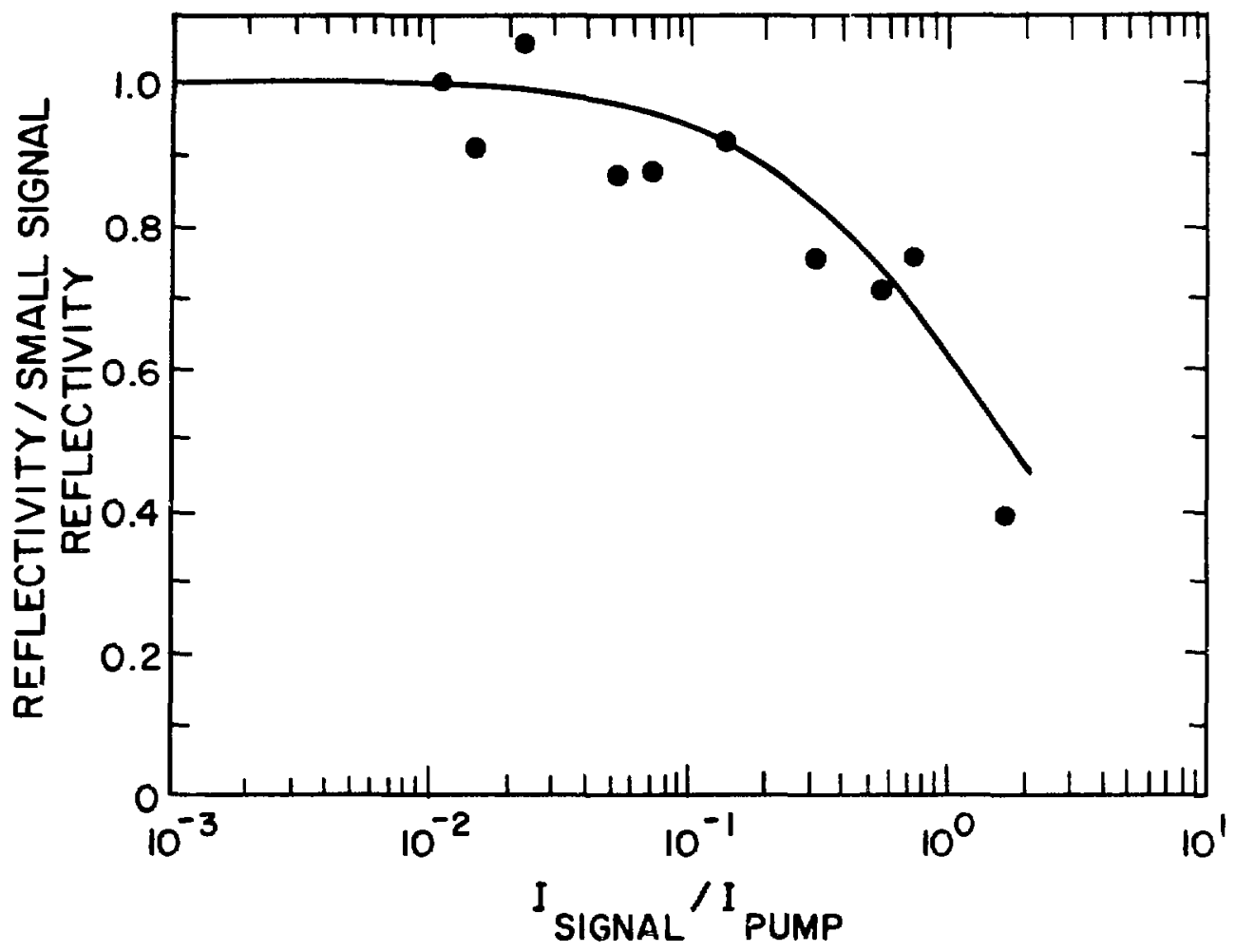

Figure III-3b. Comparisons of experimental results to theory in the regime of pump depletion. A DFWM experiment is performed at near-constant pump intensity and variable probe intensity, in a 2-cm sample of intrinsic Ge, with the laser polarized along a [111]-crystal axis. The scatter is due to variations in iaser intensity (Ipump = $53 \pm 6 \mathrm{MW} / \mathrm{cm}^{2}$ so $\left.\mathrm{SSR}=.4 \pm .1\right)$. 
variations in laser intensity of over $\pm 10 \%$. The small-signal reflectivity in this case is 0.39 . The experimental value for the effective nonlinear susceptibility is $4.5 \times 10^{-11}$ esu, in good agreement with the value obtained from Table I and Appendix B, $x_{3}=3.8 \times 10^{-11} \mathrm{esu}$.

IV. Discussion and summary

In this chapter I have presented two models of DFWM in Kerr-like media. Both theories employ the slowly varying envelope approximation. The small-signal theory employs the additional approximation that the probe intensities be much less than the pump intensities, so that pump depletion and the nonlinear index induced by the probes may be ignored. Under these conditions it is relatively simple to obtain an analytic solution for the reflectivity, Eq. 19. In the large signal case, the coupled differential equations are most conveniently solved numerically.

Experimental data for DFWM in germanium has been compared to both models and found to be in good agreement. In the small-signal limit, the data generally follow the predicted $R=\tan ^{2}(\kappa L)$ dependence below the threshold for plasma formation. In fact the only significant deviation from this model is the result of the 
short laser pulse duration invalidating the time independent theory. In the large signal case, the data and the model agree well, especially considering the large variations in reflectivity that occur from the fluctuations in laser intensity.

The reduction in reflectivity that is observed as the signal intensity is increased is the result of two causes. First, there is a significant reduction in pump intensity because of the coupling of energy out of the pump beams and into the probe beams. Second, as the pump beams are depleted by the interaction, the two counterpropagating pump beams cannot remain equal in intensity throughout the interaction length. This results in the phase-mismatch mentioned in Section $B$, and a corresponding decrease in reflectivity. This latter effect is greater in the large signal theory, since all four waves contribute to the nonlinear index experienced by a given wave. It is still expected that the first of these two causes dominates for the case presented here. However, this type of effect plays a bigger role in lossy materials, such as the resonant materials dealt with in the next few chapters, where the pump beams cannot be equal in intensity throughout the interaction length because they are absorbed by the medium as they propagate. 


\section{Footnotes}

1. N. Bloembergen, Nonlinear optics (W. A. Benjamin, Inc., New York, 1965) p. 2 .

2. For a discussion see R. R. Birss, "Property tensors in magnetic crystal classes," Proceedings of the Physical Society 79,946 (1962).

3. See, for example, D. A. B. Miller, S. D. Smith, and B. $S$. Wherret, "The microscopic mechanism of third-order optical nonlinearity in InSb, "Optics Communications $35,221(1980)$.

4. P. D. Maker and R. W. Terhune, "Study of optical effects due to an induced polarization third order in electric field strength," Physical Review 137, A801 $(1965)$.

5. The equations derived here are slightly different than those presented elsewhere (see Refs. 11, 12, and 13). This is because of incompleteness on the part of other authors and because others have assumed isotropic media.

6. See for example R. W. Hellwarth, "Generation of time-reversed wavefronts by nonlinear refraction," Journal of the Optical Society of America 67, 1 (1977).

7. A. Yariv and D. M. Pepper, "Amplified reflection, phase conjugation, and oscillation in degenerate four-wave mixing," Optics Letters 1, 16 (1977).

8. J. H. Marburger and J.F. Lam, "Effect of nonlinear index changes on degenerate four-wave mixing," Applied Physics Letters 35, 249 (1979).

9. J. Feinberg and $R$. W. Hellwarth, "Phase-conjugating mirror with continuous-wave gain," Optics Letters $\underline{5}$, $519(1980)$.

10. Some work has been done on time-dependent models of DFWM in Kerr-like media. None of these models is appropriate for the geometry and temporal variation I use here. Time-dependent probe and time-independent pump models have been given by J. H. Marburger, "Optical pulse integration and chirp reversal in degenerate four-wave mixing," Applied Physics Letters 32,372 (1970), and by W. W. Rigrod, R. A. Fisher, and B. J. Feldman, "Transient analys is of nearly 
degenerate four-wave mixing," Optics Letters 5,105 (1980). A model with all waves time dependen $\bar{t}$, but using a different geometry than here is given by $D$. A. B. Miller, "Time reversal of optical pulses by four-wave mixing," Optics Letters $\underline{5}, 300$ (1980).

11. J. J. Wynne, "Optical third-order mixing in GaAs, Ge, Si, and InAs," Physical Review 178, 1295 (1969).

12. D. E. Watkins, C. R. Phipps, Jr., and S. J. Thomas, "Determination of the third-order nonlinear optical coefficients of Ge through ellipse rotation, "Optics Letters $\underline{5}, 248$ (1980).

13. H. Hsu, "Large-signal theory of phase-conjugate backscatterings," Applied Physics Letters 34,855 (1979).

14. J. H. Marburger and J. F. Lam, "Nonlinear theory of degenerate four-wave mixing, "Applied Physics Letters 34, $389(1979)$.

15. H. G. Winful, "Optical bistability in periodic structures and in degenerate four-wave mixing," in the Digest of Technical Papers, International Conference on Excited States and Multiresonant Nonlinear Optical Processes in Solids, D. S. Chemla, Ed. (Les Edition de Physique, Orsay, France, 1981), p. 55.

16. W. W. Rigrod, private communication (1980). 


\section{CHAPTER IV}

\section{THEORY OF DEGENERATE FOUR-WAVE MIXING}

\section{IN RESONANTLY ENHANCED MEDIA}

\section{Introduction}

The idea of resonantly enhancing a nonlinear optical process is quite common. A well known example where this idea plays a fundamental role is Raman spectroscopy. In the case of degenerate four-wave mixing (DFWM), two somewhat distinct types of resonant enhancement have been investigated. These are the resonant enhancement that occurs near the band gap in semiconductors and resonant enhancement due to saturable absorption. The first of these is discussed in more detail in Chapter VI. The second is the subject of this chapter, and of Chapters $V$ and VII.

Experimental results in resonantly enhanced DFWM have been reported in sodium vapor, 1 rubidium vapor, ${ }^{2}$ ruby crystal, ${ }^{3}$ and inverted $N d: Y A G,{ }^{4}$ in several semi-conductors near the band gap, 5,6,7 in numerous infrared absorbing gases, ${ }^{8,9}$ and in inverted $\mathrm{CO}_{2} \cdot 10$ Also, I have reported ${ }^{11}$ the first quantitative comparison of resonantly enhanced DFWM in $\mathrm{KCl}: \mathrm{KReO}_{4}$ with the theory of Abrams and Lind $^{12}$ (see Chapter VII). These experimental results were the original motivation for the study presented here. 
In this chapter, I present a model of DFWM in a two-level homogeneously broadened saturable absorber. The model is an extension of the original work by Abrams and Lind ${ }^{12}$ to include the effect of pump attenuation by the medium. The general formulation of this model provides a basis for developing the specialized models used in later chapters to analyze DFWM in p-type germanium and in $\mathrm{KCl}: \mathrm{KReO}_{4}$. The more sophisticated model developed here is compared to the Abrams and Lind model under conditions of significant pump absorption, and to a model that assumes a broadband inhomogeneously broadened saturable absorption rather than homogeneous broadening.

The model presented here is a generalization of the original work of Abrams and Lind. The model given by Abrams and Lind has three underlying assumptions. 12 First there is the slowly varying envelope approximation (SVEA) which underlies essentially all such theories of nonlinear optical interactions. Second, the probe intensities are assumed to be much weaker than the pump intensities, so that a perturbation analysis can be used. The third assumption Abrams and Lind make is that the pump beams are constant throughout the interaction volume. They somewhat mistakenly refer to this as assuming no pump depletion. This would follow froin the weak probe assumption. In fact, this assumption also requires that 
the pump beams are not substantially attenuated by the medium so that either the optical frequency must be substantially detuned from resonance or the absorption on resonance must be very weak. It is this third assumption that is removed in the present analysis.

A. Theory of DFWM in a two-level homogeneously broadened saturable absorber

Both the Abrams and Lind model and the model presented here are based on a perturbation expansion of the well known polarizability for a two-level homogeneously broadened system to first order in the weak probe fields. This first order polarizability is used in the wave equation to obtain separate coupled equations for the two pump waves and the two probe waves. In Abrams and Lind's solution, the equations for the pump beams are completely ignored, and the probe wave equations are solved for constant pump intensity. In the solution developed here both sets of equations are solved numerically.

The polarizability of a two-level system is given in terms of the total field $E(\nu)$ by ${ }^{12}$

$$
P(\nu)=\frac{\alpha_{0}}{2 \pi k_{0}} \frac{(i-\delta) E}{\left(1+\delta^{2}+|E|^{2} / E_{s}^{2}\right)} .
$$


Here $\alpha_{0}=N \pi|\mu|^{2} T_{2} k_{0} / h$ is the line-center small-signal field absorption coefficient, $E_{s}^{2}=n / T_{1} T_{2}|\mu|^{2}$ is the saturation field, $\delta=\left(\nu-\omega_{0}\right) T_{2}$ is the normalized detuning of the laser from the resonant transition frequency $\omega_{0}$, and $k_{0}$ is the free space wave number. The dipole moment is $\mu$ and $\mathrm{T}_{1}$ and $\mathrm{T}_{2}$ are the longitudinal and transverse (energy and phase) relaxation times respectively. The total field $E(v)$ is the sum of the four interacting waves, and $I$ use the notation $E(r, t)=\operatorname{Re}\left(E(v) e^{-i v t}\right)$. Following Abrams and Lind, I write the normalized sum of the pump fields and the probe fields as $E_{0}$ and $\Delta E$ respectively, so $E / E_{S}=E_{0}+\Delta E$. The polarizability of $E q$. 1 to first order in $\Delta E$ and $\Delta E^{*}$ is

$$
\begin{aligned}
& P=P_{0}+\Delta P=\frac{\alpha_{0} E_{s}}{2 \pi k_{0}} \frac{(i-\delta) E_{0}}{\left(1+\delta^{2}+\left|E_{0}\right|^{2}\right)} \\
& +\frac{\alpha_{0} E_{s}}{2 \pi k_{0}}(i-\delta) \frac{\left(1+\delta^{2}\right) \Delta E-E_{0}^{2} \Delta E^{\star}}{\left(1+\delta^{2}+\left|E_{0}\right|^{2}\right)^{2}} .
\end{aligned}
$$

The full wave equation is

$$
\nabla^{2} E(\nu)+\frac{\nu^{2}}{c^{2}} E(\nu)=-\frac{4 \pi v^{2}}{c^{2}} P(\nu) .
$$


Separating zero- and first-order terms in Eq. 3 gives

$$
\begin{aligned}
& \text { (4a) } \quad \nabla^{2} E_{0}+\frac{\nu^{2}}{c^{2}} E_{0}=-\frac{4 \pi v^{2}}{c^{2} E_{s}} P_{0} \\
& \text { (4b) } \quad \nabla^{2} \Delta E+\frac{\nu^{2}}{c^{2}} \Delta E=-\frac{4 \pi \nu^{2}}{c^{2} E_{s}} \Delta P \text {. }
\end{aligned}
$$

These equations are to be treated in the SVEA. For the left hand side of this equation, the procedure is to substitute $E_{0}=E_{f} \exp \left(i k_{0} \zeta\right)+E_{b} \exp \left(-i k_{0} \zeta\right)$ and $\Delta E=E_{1} \exp \left(i k_{0} \zeta_{1}\right)+E_{2} \exp \left(-i k_{0} \zeta_{1}\right)$ and use the procedure ${ }^{13}$ given in Chapter III. To obtain a slowly varying form for the right hand side is more difficult. This is because the counter-propagating pump beams set up a standing wave in the medium. The response of the medium to this standing wave is a spatial variation in the polarizability at the wavelength of the standing wave, that is at $\lambda / 2$. To remove this rapid variation from the polarizability, an average is taken over a single period of the standing wave. This average is taken for the coefficient of $E_{0}$ in the expression for $P_{0}$ and for the coefficients of $\Delta E$ and $\Delta E^{*}$ in the expression for $\Delta P$. After taking this average, I can substitute the results into Eqs. $4 a$ and $b$ and equate coefficients of $\exp \pm(j k z)$ to obtain the four equations

(5a) $\quad \frac{d E_{f}}{d z}=-\alpha_{p} E_{f}$, 
(5b)

$$
\frac{d E_{b}}{d z}=\alpha_{p} E_{b},
$$

(5c) $\frac{d E_{1}}{d z}=\alpha E_{1}+i k^{*} E_{2} * \exp \left(i \phi_{+}\right)$,

and

$$
\frac{d E_{2}}{d z}=-\alpha E_{2}-i \kappa^{*} E_{1} * \exp \left(i \phi_{+}\right) .
$$

Here

$$
\alpha_{p}=\frac{\alpha_{0}(1+i \delta)}{\cos (\theta) D},
$$

(6b)

$$
\alpha=\frac{-\alpha_{0}(1+i \delta)\left(1+\delta^{2}\right)\left(1+\delta^{2}+\rho_{f}^{2}+\rho_{b}^{2}\right)}{\cos (\theta) D^{3 / 2}},
$$

$$
\kappa=\frac{\alpha_{0}(i+\delta)\left(1+\delta^{2}\right) 2 \rho_{f} \rho_{b}}{\cos (\theta) D^{3 / 2}} \text {, }
$$

$\phi_{+}=\phi_{f}+\phi_{b}$ is the sum of the pump beam

phases, and

$$
D=\left(1+\delta^{2}\right)^{2}+2\left(1+\delta^{2}\right)\left(\rho_{f}{ }^{2}+\rho_{b}{ }^{2}\right)+\left(\rho_{b}{ }^{2}-\rho_{f}{ }^{2}\right)^{2} .
$$

The amplitudes $\rho_{f}=\left|E_{f}\right|$ and $\rho_{b}=\left|E_{b}\right|$.

Equations 5 and 6 reduce to Abrams and Lind's equa-

tions under the conditions that they assumed, specifical-

$l y$, that $\rho_{f}=\rho_{b}$ are constant. In this particular case, $\phi_{+}$is a constant. This is true in general if $\rho_{f}=\rho_{b}$, true here independent of this condition, but not true in later problems. In the solution that I derive here, I will retain this term for the sake of generality. Abrams and Lind arbitrarily, but without loss 
of generality, put $\phi_{+}=0$. In their case the constancy of the pump intensities gives constant coupling coefficients in the coupled probe wave equations. Given this, Abrams and Lind are able to solve the coupled Eqs. $5 c$ and d analytically. They find that the reflectivity, or the ratio of the "reflected" intensity to the input signal intensity is given by ${ }^{12}$

$$
R=\frac{|\kappa \sin (w L)|^{2}}{\left|w \cos (w L)+\alpha_{R} \sin (w L)\right|^{2}}
$$

where $w=\sqrt{|\kappa|^{2}-\alpha_{R}^{2}}, \alpha_{R}=\operatorname{Re}(\alpha)$ and $L$ is

the length of the sample. This solution is approximate in that it ignores attenuation of the pump beams and the effect of phase shifts introduced by the nonlinear index of refraction of the medium. It is a good approximation in the limit of very weak absorption. It is also a good approximation in the limit of very large pump beam intensicies, where the transition is highly saturated and little absorption occurs.

Another analytic solution of the coupled Eqs. $5 \mathrm{c}$ and $d$ has been given by $W$. W. Rigrod. ${ }^{14}$ In this solution Eqs. $5 \mathrm{c}$ and d are transformed to an equivalent second order differential equation of the form

$$
\frac{d^{2} B_{2}}{d z^{2}}+w^{2} B_{2}=0
$$


where

$$
\text { (9) } \begin{aligned}
w^{2}= & \mid k^{2}-\alpha_{R}^{2}+\frac{1}{4}\left(\frac{d \phi+}{d z}\right)^{2}+\frac{\alpha_{R}}{k^{*}} \frac{d k^{*}}{d z}+\frac{1}{2 k^{*}} \frac{d^{2} k^{*}}{d z^{2}} \\
- & \frac{3}{4} \frac{1}{k^{*} 2}\left(\frac{d k^{*}}{d z}\right)^{2}-\frac{d \alpha_{R}}{d z}+i\left\{\alpha_{R} \frac{d \phi_{*}}{d z}+\frac{1}{2} \frac{d^{2} \phi_{+}}{d z^{2}}\right. \\
& \left.-\frac{1}{2 k^{*}} \frac{d k^{*}}{d z} \frac{d \phi+}{d z}\right\} .
\end{aligned}
$$

The quantity $w$ is a complex function of distance, $z$, because $\alpha, \kappa$, and $\phi_{+}$are in general functions of $z$. Nevertheless, Eq. 8 can be solved in the WKB approximation. The solution in this approximation is

where

$$
R=\left|\frac{|\kappa(0)| \sin (\bar{w} L)}{w(0) \cos (\bar{w} L)+\left(\alpha_{R}(0)-\frac{i}{2} \frac{d \phi+}{d z}(0) \sin (\bar{w} L)\right.}\right| 2
$$

$$
\bar{w}=\frac{1}{L} \int_{0} L w(z) d z,
$$

and both $w$ and $w$ are generally complex. The WKB approximation used in this solution is valid under conditions such as

$$
\text { (11) }\left|\frac{d k}{d z}\right| \ll|k|^{2} \text {. }
$$

Investigation has shown that such conditions are met for pump intensities $I_{\text {pump }} \ll C I_{s}$, where $C$ is a constant on the order of unity and $I_{S}$ is the saturation intensity. 
Although a solution to Eqs. $5 \mathrm{c}$ and $d$ has been obtained analytically, the complete solution is only semianalytical, because no such solution exists for the coupled pump Eqs. $5 \mathrm{a}$ and $\mathrm{b}$. These equations must still be solved numerically.

The two solutions to Eqs. 5 discussed so far cover the two extreme cases $I \ll I_{S}$ and $I \gg I_{S}$. For intermediace intensities, I $\mathrm{I}_{s}$, the best solution is a numerical treatment of the couplad differential equations.

To obtain a numerical solution for Eqs. 5, I note that the pump equations are complete?y decoupled from the probe equations. Substituting for the pump beams $E_{j}=\rho_{j} \exp \left(i \phi_{j}\right)$, I obtain

$$
\begin{aligned}
& \text { (12a) } \frac{d \rho_{f}}{d z}=-\alpha_{p R} \rho_{f} \\
& \text { (12b) } \frac{d \rho_{b}}{d z}=-\alpha_{p R} \rho_{b}
\end{aligned}
$$

where $\alpha_{p}=\alpha_{p R}+i \alpha_{p I}$, and

$$
\text { (12c) } \quad \frac{d \phi+}{d z}=0
$$

or $\phi_{+}$is constant, in this particular case. In order to obtain the most general form for numerical computation in later cases, I will assume that, in general, $\phi_{+}$is not constant, and retain its derivatives here. Note that 
$\alpha_{p}$ is independent of the phases $\phi_{f}$ and $\phi_{b}$.

This result is general, and is a consequence of the

spatial averaging process that is used to obtain $\alpha_{p}$ in the SVEA. In the unaveraged form for $\alpha_{p}$, the phases occur as the difference $\phi_{-}=\phi_{f}-\phi_{b}$. The important parameter is the sum $\phi_{+}$, which, as in the small signal solution for Kerr-like media in the previous chapter, measures the effect of the nonlinear index of refraction on the propagation of the pump waves. Since the pump bealis are being scattered to generate the reflected beam, if changes in the pump beam phases are substantial, the phase of the scattered pump beam will vary relative to that of the wave being generated. This introduces a phase-mismatch, so that destructive interference can occur, reducing the overall efficiency of the wave mixing process.

An analytical solution to Eqs. 12 a and $b$ is not known. It is, however, relatively simple to solve the coupled equations numerically. The value of $\rho_{f}$ is given at the boundary $z=0$. The value of $\rho_{b}$ is given at the opposite boundary, $z=L$. An iterative process must be used to obtain the solution, where the value of the amplitude $\rho_{b}$ is guessed at the $z=0$ boundary, and the coupled waves are used to generate a solution. The value $\rho_{b}(L)$ is compared to the boundary condition, and 
used to improve the guess of $\rho_{b}(0)$. This process continues until it converges. The numerical routine that I developed for this process normally converges in two to four iterations to a value within $0.1 \%$ of the boundary condition. This is quite adequate for my purposes.

To obtain a form of the coupled probe-wave equations that is convenient for numerical integration, I first note that the phase shift introduced by the imaginary part of $\alpha$ is common to both waves. Transforming the Eqs. $5 \mathrm{c}$ and d by

$$
E_{2}=A_{1} \exp \pm i \int_{0}^{Z} \alpha_{I}(x) d x
$$

where $\alpha=\alpha_{R}+i \alpha_{I}$, removes $\alpha_{I}$ from the coupled equations:

$$
\text { (14a) } \frac{d A_{1}}{d z}=\alpha_{R} A_{1}+i k * A_{2} * \exp \left(i \phi_{+}\right)
$$

and

(14b) $\frac{d A_{2}}{d z}=-\alpha_{R} A_{L}-i k^{*} A_{1} * \exp \left(i \phi_{+}\right)$.

Now put $\kappa^{*}=\kappa_{R}-i \kappa_{I}$ and $A_{j}=\rho_{j} \exp \left(i \phi_{j}\right)$ and define the total phase $\phi=\phi_{+}-\phi_{1}-\phi_{2}$. Separating real and imaginary parts gives

(15a) $\frac{d \rho_{1}}{d z}=\alpha_{R} \rho_{1}-\left(\kappa_{R} \sin (\phi)-\kappa_{I} \cos (\phi)\right) \rho_{2}$

(15b) $\frac{d \rho_{2}}{d z}=-\alpha_{R} \rho_{2}+\left(\kappa_{R} \sin (\phi)-\kappa_{I} \cos (\phi)\right) \rho_{1}$ 
$(15 c) \quad \rho_{1} \frac{d \phi 1}{d z}=\left(k_{R} \cos (\phi)+\kappa_{I} \sin (\phi)\right) \rho_{2}$
$(15 d) \quad \rho_{1} \frac{d \phi 2}{d z}=-\left(\kappa_{R} \cos (\phi)+\kappa_{I} \sin (\phi)\right) \rho_{1}$.

I choose $\rho_{2}$ as the injected prote wave, with known input value at the boundary $z=L$. Then $\rho_{1}$ is the generated wave with the boundary condition $\rho_{1}(z=0)=0$. From Eq. 15c, this boundary condition implies

$$
\tan (\phi(0))=\frac{-k_{R}(0)}{K_{I}(0)} \text {. }
$$

For $z \neq 0$ the equation for the total phase is

$$
\frac{d \phi}{d z}=\frac{d \phi+}{d z}-\left(K_{R} \cos (\phi)+\kappa_{I} \sin (\phi)\right)\left(\frac{\rho_{2}}{\rho_{1}}-\frac{\rho_{1}}{\rho_{2}}\right)
$$

The coupled Eqs. $15 \mathrm{a}$ and $\mathrm{b}$ and 17 form the basis for the numerical solution of the probe waves, using the solution of the pump waves discussed earlier. The only approximations in this solution are the slowly varying envelope approximation and the weak probe approximation. The quanticis of interest is the reflectivity, $R=\left(\rho_{1}(L) / \rho_{2}(L)\right)^{2}$.

B. DFWM in an inhomogeneously broadened medium

Equations 5 and the solutions presented in the previous section are quite general, and apply whenever the polarizability of the medium is expanded to only first 
order in the weak probe fields. The coefficients $a_{p}$, $\alpha$, and $k$ vary according to the polarizability used. (These equations even apply to the small signal theory of the previous chapter. In that case $\alpha_{p}$ is imaginary and gives the phase mismatch for the pump beams, $\alpha$ is also imaginary, and so vanishes from the problem, and $k$ is the same as in chapter III. The two solutions given by Eq. 10 of this chapter and Eq. 19 of the previous chapter can be shown to be equivaient.) Thus for all future problems, it is sufficient to determine the coefficients $\alpha_{p}, \alpha_{R}$, and $k$ and then apply the appropriate solution given in section A.

For instance, the polarizability of a broadband inhomogeneously broadened saturable absorber $c a n$ be obtained from Eq. 1 by integrating over $\delta$ from $-\infty$ to $\infty .15$ This essentially gives an ensemble of absarbers of uniform strength and density for all wavelengths. The result is

$$
P_{I H}=\frac{i \alpha_{0}}{2 \pi k_{0}} \frac{E}{\left(1+|E|^{2} / E_{s}\right)^{\frac{1}{2}}} .
$$

Expanding this polarizability to first order in the weak field $\triangle E, I$ obtain

$$
P_{I H}=P_{O I H}+\Delta P_{I H}
$$


(19)

$$
\begin{aligned}
& =\frac{i \alpha_{0} E_{S}}{2 \pi k_{0}} \frac{E_{0}}{\left(1+\left|E_{0}\right|^{2}\right)^{\frac{1}{2}}} \\
& +\frac{i \alpha_{0} E_{S}}{2 \pi k_{0}} \frac{\left(2+\left|E_{0}\right|^{2}\right) \Delta E-E_{0}^{2} \Delta E^{*}}{\left(1+\left|E_{0}\right|^{2}\right)^{3 / 2}} .
\end{aligned}
$$

From this equation, I obtain the coefficients $\alpha_{p}$, $\alpha$, and $k$ from the spatial averages (for the SVEA) of the terms multiplying $E_{0}, \Delta E$ and $\Delta E *$ respectively. In this case, the spatial averages involve complete elliptic integrals of the first and second kind with argument $b=4 \rho_{f} \rho_{b} /\left(1+\left(\rho_{f}+\rho_{b}\right)^{2}\right)$. The coefficients are

$$
\alpha_{p}=\frac{\alpha_{0}}{\pi \cos (\theta)\left(1+\left(\rho_{f}+\rho_{b}\right)^{2}\right)^{\frac{T}{2}}} K(b)
$$

$$
\begin{aligned}
& \text { (21) } \alpha=\frac{-\alpha_{0}}{\pi \cos (\theta)\left(1+\left(\rho_{f}+\rho_{b}\right)^{2}\right)^{\frac{1}{2}}}\left\{k(b)+\frac{E(b)}{1+\left(\rho_{f}-\rho_{b}\right)^{2}}\right\} \\
& \text { (22) } k=\frac{i \alpha_{o}}{\pi \cos (\theta)\left(1+\left(\rho_{f}+\rho_{b}\right)^{2}\right)^{\frac{2}{2}}} \times \\
& \quad\left\{\frac{\rho_{f}^{2}+\rho_{b}{ }^{2}}{2 \rho_{f} \rho_{b}}[K(b)-E(b)]-\frac{\left(\rho_{f}-\rho_{b}\right)^{2}}{1+\left(\rho_{f}-\rho_{b}\right)^{2}} E(b)\right\}
\end{aligned}
$$

These coefficients are used here for a comparison of DFWM in inhomogeneously broadened versus homogeneously broadened media. In the next chapter, they are incorporated into a more general form for the polarizability of p-type germanium. 
C. Discussion and conclusions

In this chapter I have presented a numerical technique for the solution of the coupled wave equations describing DFWM in two-level homogeneously broadened media and broadband inhomogeneously broadened media. I have also presented the original solution of the problem given by Abrams and Lind, which ignored pump attenuation by the medium. In this section I graphically compare these results.

Figure 1 compares the reflectivity as a function of pump intensity predicted by the two models. The solid lines are the Abrams and Lind model, and the dashed lines are my model. This figure is analogous to Fig. 2 of Abrams and Lind's erratum, ${ }^{16}$ and $I$ use the same values of $\alpha_{0} L$. These calculations are done on resonance, where the effect of pump attenuation by a given medium will be most severe. As seen in the case of $\alpha_{0} L=l$, Abrams and Lind's model is good for large pump intensities where the medium is highly bleached and the effective attenuation of the pump beams is small. The trend of improvement in Abrams and Lind's model with decreasing $\alpha_{0} \mathrm{~L}$ can also be seen in the figure. For $\alpha_{0} \mathrm{~L}=6$, this plot shows Abrams and Lind's prediction to be an overestimate of the reflectivity by as much as a factor of 1000. This is because such a sample has a small-signal 


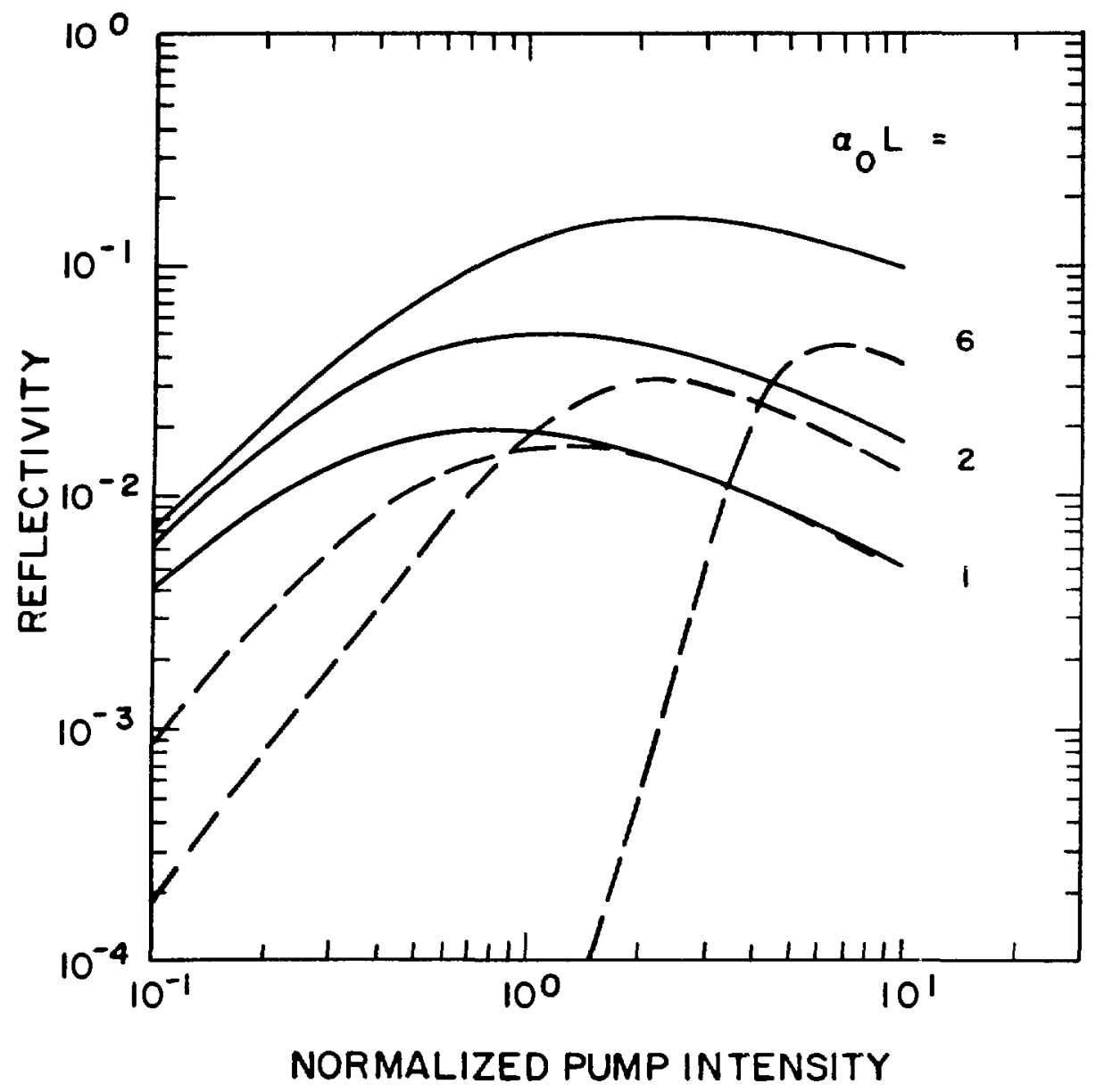

Figure IV-1. Comparison of DFWM models with (dashed curves) and without (solid curves) pump attenuation by the medium for $\delta=0$. The three solid lines are reproductions of Abrams and Lind's curves (see Ref. 16). The dashed lines are the numerical model presented in the text, for the same cases. The failure to include pump attenuation effects results in a gross overestimation of the reflectivity. 
intensity transmission of on 1 y $6 \times 10^{-6}$, and bleaches very slowly. Until it is bleached, no wave mixing can occur because the pump beams are effectively reduced to zero by the medium. Abrams and Lind went so far as to calculate the case $\alpha_{0} L=14$ (small-signal transmission of $7 \times 10^{-13}$ ). Clearly such a calculation which ignores the effect of pump attenuation is absurd.

As can be seen from the dashed curves of $F i g .1$, the general effects of an increase in absorption coefficient are a shift of the peak reflectivity toward highor intensity, an increase in pump intensity dependence below the peak (from less than a square dependence to greater than a cubic dependence), and no significant increase or decrease in the peak reflectivity. Eventually, as $\alpha_{0} L$ is increased or decreased there is a significant decrease in peak reflectivity, tither because the resonant effect becomes very weak, or, by the time the pump intensity is great enough to bleach the medium, it is so intense throughout most of the medium that the coupling coefficient is also reduced to nothing.

Figure 2 is analogous to Abrams and Lind's Fig. 3.16 In this case, the small-signal intensity transmission is held constant at $13.5 \%$ as the optical frequency is detuned from resonance. Thus the small-signal absorption coefficient on resonance, $\alpha_{0} L$, must be increased with 


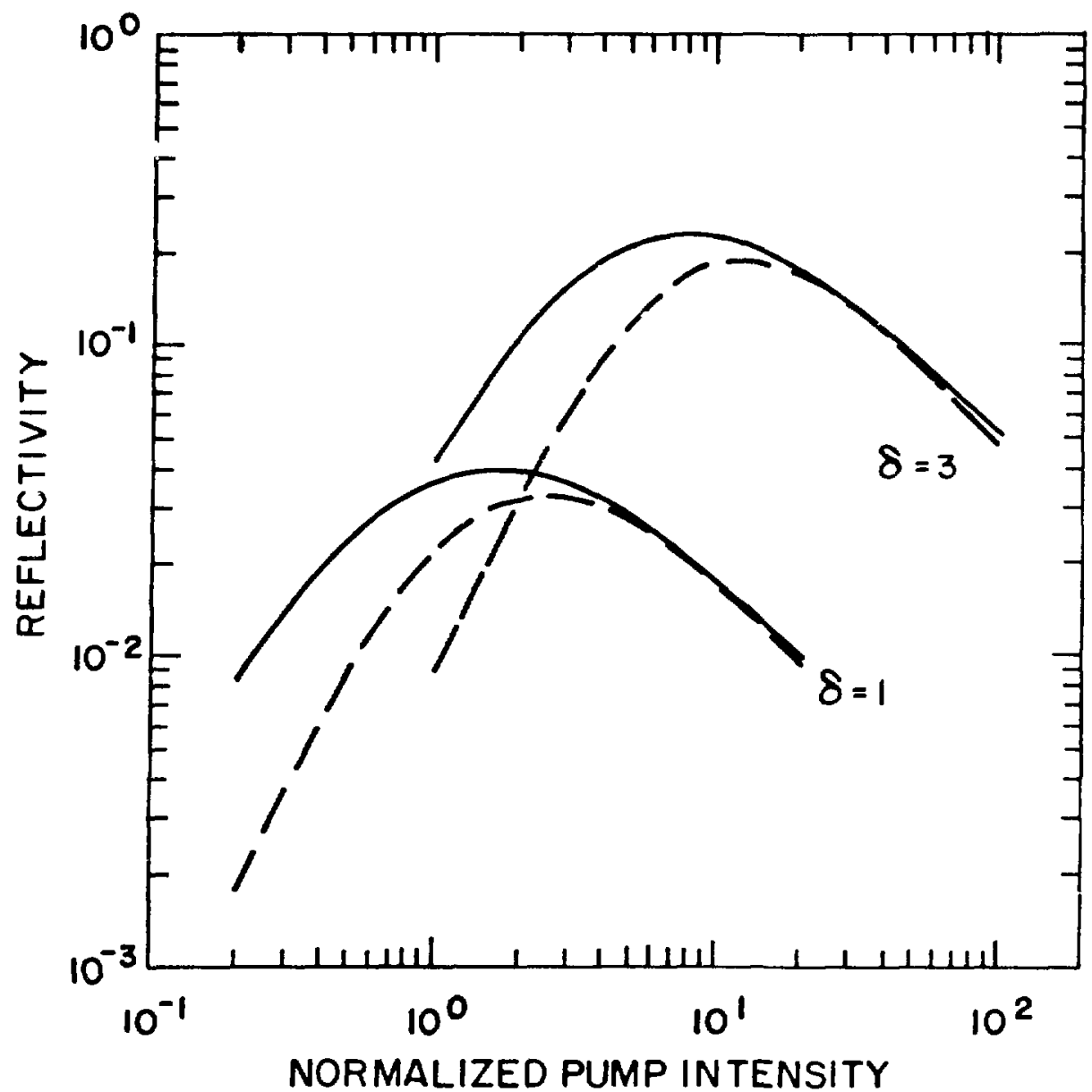

Figure IV-2. Comparison of DFWM models with (dashed lines) arid without (solid lines) pump attenuation as a function of detuning, $\delta$, for constant attenuation corresponding to a line center attenuation of $\alpha_{0} L=1$. The solid curves are a reproduction of Abrams and Lind's curves (see Ref. 16). In this case, the attenuation of the pump beams is always the same, so the degradation in reflectivity is similar (see also the $\alpha_{0} L=1, \delta=0$ case in Fig. 1). By going to larger detuning $\delta>10$, amplified reflection is possible. 
increased detuning. The curves plotted here are analogous to the $\alpha_{0} L=1$ curve of Fig. 1. Indeed, in the two cases plotted here, my model and the Abrams and Lind model converge in essentially the same way as in the $\alpha_{0} L=1$ case of Fig. 1. The increase in peak reflectivity with increased detuning under these circumstances continues and eventually gain (or reflectivity greater than unity) is predicted.

Finally, in Fig. 3, I compare DFWM in inhomogeneously broadened media (dashed line) to homogeneous media (solid line) with the same small-signal transmission. The inhomogeneous curve is broader and its peak is less than that for the homogeneous case. Both of these features arise from of the slower bleaching of inhomogeneous media with intensity.

In conclusion, I have presented a numerical technique for the solution of the coupled differential equations which describe DFWM in saturable absorbers. The numerical solution includes the effect of attenuation of the pump beams by the medium and nonlinear index effects induced by the pump beams. I have compared my solution to a published solution ${ }^{12}$ which ignores these effects, and found the published solution to be inadequate. I have also compared DFWM in homogeneously broadened media to DFWM in inhomogeneously broadened media. The coupled 


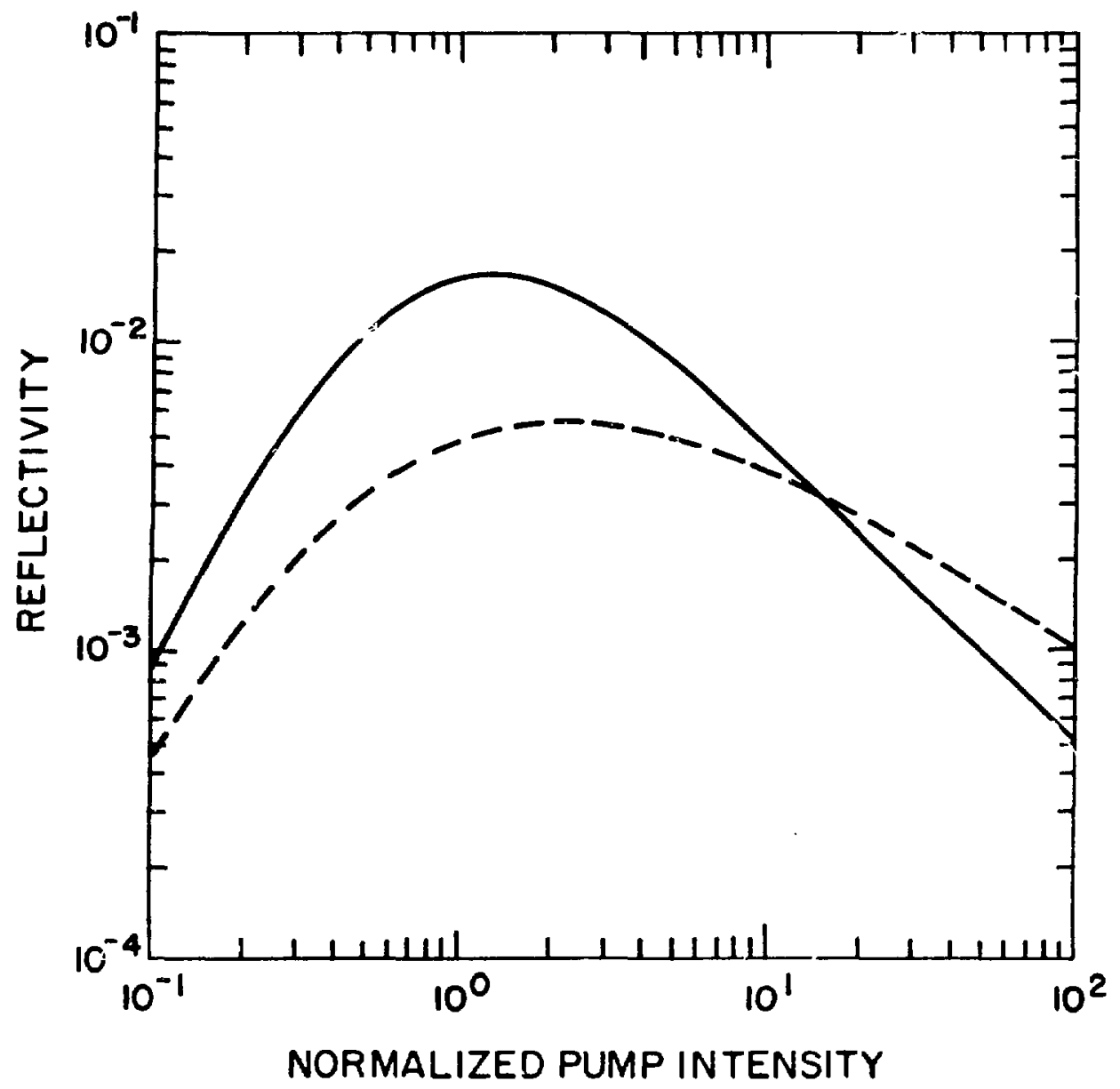

Figure IV-3. Comparison of DFWM models for homogeneousiy broadened media on resonance (solid curve) to inhomogeneously broadened media (Jashed curve). The inhomogeneously broadened model is wider and does not reach as great a peak reflectivity. This is the result of the slower bleaching of inhomogeneous media. Both curves are for $\alpha_{0} L=1$. 
differential equations describing DFWM and the numerical technique for their solution presented here are completely general, and are applied to the description of DFWM in $\mathrm{p}$-type Ge and $\mathrm{KCl}: \mathrm{KReO}_{4}$ in later chapters. 


\section{Footnotes}

1. D. M. Bloom, P, F. Liao, and N. P. Economou, "Observation of ampified degenerate four-wave mixing in atomic sodium vapor," Optics Letters 2, 58 (1978).

2. D. Grischkowsky, N. S. Shiren, and R. J. Bennett, "Generation of time reversed wave fronts using a resonantly enhanced electric nonlinearity, "Applied Physics Letters 33,805 (1978).

3. P. F. Liao and D. M. B $100 \mathrm{~m}$, "Cont inuous-wave backward-wave generation by degenerate four-wave mixing in ruby," Optics Letters $\underline{3}, 4$ (1978).

4. A. Tomita, "Phase conjugation using gain saturation of a Nd:YAG laser," Applied Physics Letters 34, 463 (1979).

5. For results in Si, see J. P. Woerdman, "Formation of a transient free-carrier hologram in Si," Optics Communications 2, 212 (1971), or R. K. Jain, M. B. Klein, and R. C. Lind, "High-efficiency degenerate four-wave mixing of $1.06 \mu \mathrm{m}$ radiation in silicon," 0ptics Letters 4 , 328 (1979).

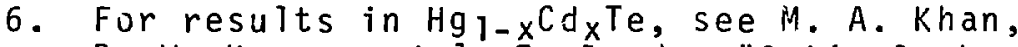
P. W. Kruse and J. F. Ready, "Optical phase conjugation in $\mathrm{Hgl}_{-\mathrm{x}} \mathrm{Cd}_{\mathrm{x}} \mathrm{Te}$," 0 ptics Letters 5,26 ? (1980) or R. K. Jain and D. G. Steel, "Degeñerate four-wave mixing of $10.6 \mu \mathrm{m}$ radiation in $\mathrm{Hg}_{-\mathrm{x}} \mathrm{Cd}_{\mathrm{x}} \mathrm{Te}$," Applied Physics Letters 37 , I (1980).

7. Resonant DFWM near the bandgap in InSt ${ }^{2}$ as been reported by D. A. B. Miller, R. G. Harrison, A. M. Johnston, C. T. Seaton, and S. D. Smith, "Degenerate four-wave mixing in InSb at $5^{\circ} \mathrm{K}$ " optics Communications 32,478 (1980). Forward DFWM near the bandgap in InAs has recently been observed by D. C. Haueisen and D. Depatie, private communication.

8. A. Elci, D. Rogovin, D. Depatie, and D. Häueisen, "Phase conjugation in ammonia," Journa! c.l: the 0ptical Society of America 70, 990 (1980).

9. R. C. Lind, D. G. Steel, M. B. Klein, R. L. Abrams, C. R. Guiliano, and R. K. Jain, "Phase conjugation ac $10.6 \mu \mathrm{m}$ by resonantly enhanced degenerate fuur-wave mixing," Applied Physics Letters 34 , 457 (1979). 
10. R. A. Fisher and B. J. Feldman, "On-resonant phase-conjugate reflection and amplification at $10.6 \mu \mathrm{m}$ in inverted $\mathrm{CO}_{2}$," Optics Letters 4,140 (1979).

11. D. E. Watkins, J. F. Figueira, and S. J. Thomas, "Observation of resonantly enhanced degenerate four-wave mixing in doped alkali halides," Optics Letters $5,169(1980)$.

12. R. L. Abrams and R. C. Lind, "Degenerate four-wave mixing in absorbing media," Optics Letters 2, 94 (1978), and Erratum, 0ptics Letters 3 , 205 T1978).

13. See Chapter III. Eqs. 9 and 10. Note that I use the same symmetry arguments here as in Chapter III to reduce the equations so that they involve only derivatives with respect to $z$, that is

$$
\frac{d}{d x} \text { and } \frac{d}{d y} \rightarrow 0 \text {. }
$$

14. W. W. Rigrod, private communication.

15. This polarizability is used in the next chapter and is the basis of several people's work in p-type Ge. See for example, F. Keilmann, "Infrared saturation spectroscopy in p-type germanium, "IEEE Journal of Quantum Electronics, QE-12, 592 (1976) and C. R. Phipps, Jr. and S. J. Thomas, "Saturation behavior of p-type germanium at $\mathrm{CO}_{2}$ laser wavelengths," Optics Letters 1, 93 (1977).

16. R. L. Abrams and R. C. Lind, Erratum, Optics Letters 3, 205 (1978). 


\section{CHAPTER $V$}

\section{DFWM IN P-TYPE GERMANIUM}

\section{Introduction}

In the pr vious chapter, models for DFWM in saturable absorhers have been presented. In this chapter, these models are adapted to the specific case of DFWM in p-type Ge. This material is particularly interesting because three different mechanisms for DFWM can be observed. First, p-type Ge exhibits saturable absorption, and thus can be used for resonantly enhanced DFWM. This mechanism dominates at low pump intensities. Second, there is the third-order nonlinear susceptibility inherent to Ge as discussed in Chapter III. From my previous work with intrinsic Ge, the magnitude of this interaction is well known. This mechanism plays a major role at intermediate intensitics, where it gives a contribution to the wave mixing process of about the same magnitude as the resonantly enhanced process. This mechanism also introduces a nonlinear index of refraction, which can cause a phase mismatch and reduce the efficiency.

Finally, there is the mechanism of free-carrier plasma generation, which dominates at very high intensities. This last mechanism is discussed in detail in the next chapter. Here, it is the interplay of the first two mechanisms that is of particular interest. 
This chapter first gives a description of p-type Ge as a saturable absorber. Then, in the second section, the polarizability is obtained from the absorption characteristics and the third-order nonlinear susceptibility. This polarizability is expanded in a perturbation series in the weak probe waves, as in the previous chapter. Given this expansion, the coefficients $\alpha_{p}, \phi_{+}, \alpha$ and $k$ can be obtained. The models of Chapter III can then be applied. In the third section, these models are compared to the results of DFWM experiments in $p$-type Ge, and a discussion of the results is given in the last section.

\section{A. P-type Ge as an inhomogeneously broadened saturable} absorber

A simplified diagram ${ }^{1}$ of the valence structure of p-type Ge is given in Fig. 1. When Ge is doped p-type, a thermal distribution of holes appears in the heavy-and light-hole bands (the lowest band is too far removed to be significantly populated at normal temperatures). optically induced transitions from the heavy- to light-hole bands are dipole allowed in the $\vec{k} \cdot \vec{p}$ approximation away from zero wave vector. ${ }^{2}$ The arrow in the diagram shows the transition for optical wavelength $\lambda=10.6 \mu \mathrm{m}\left(\mathrm{P}-20\right.$ line of the $\mathrm{CO}_{2}$ laser $)$. The 


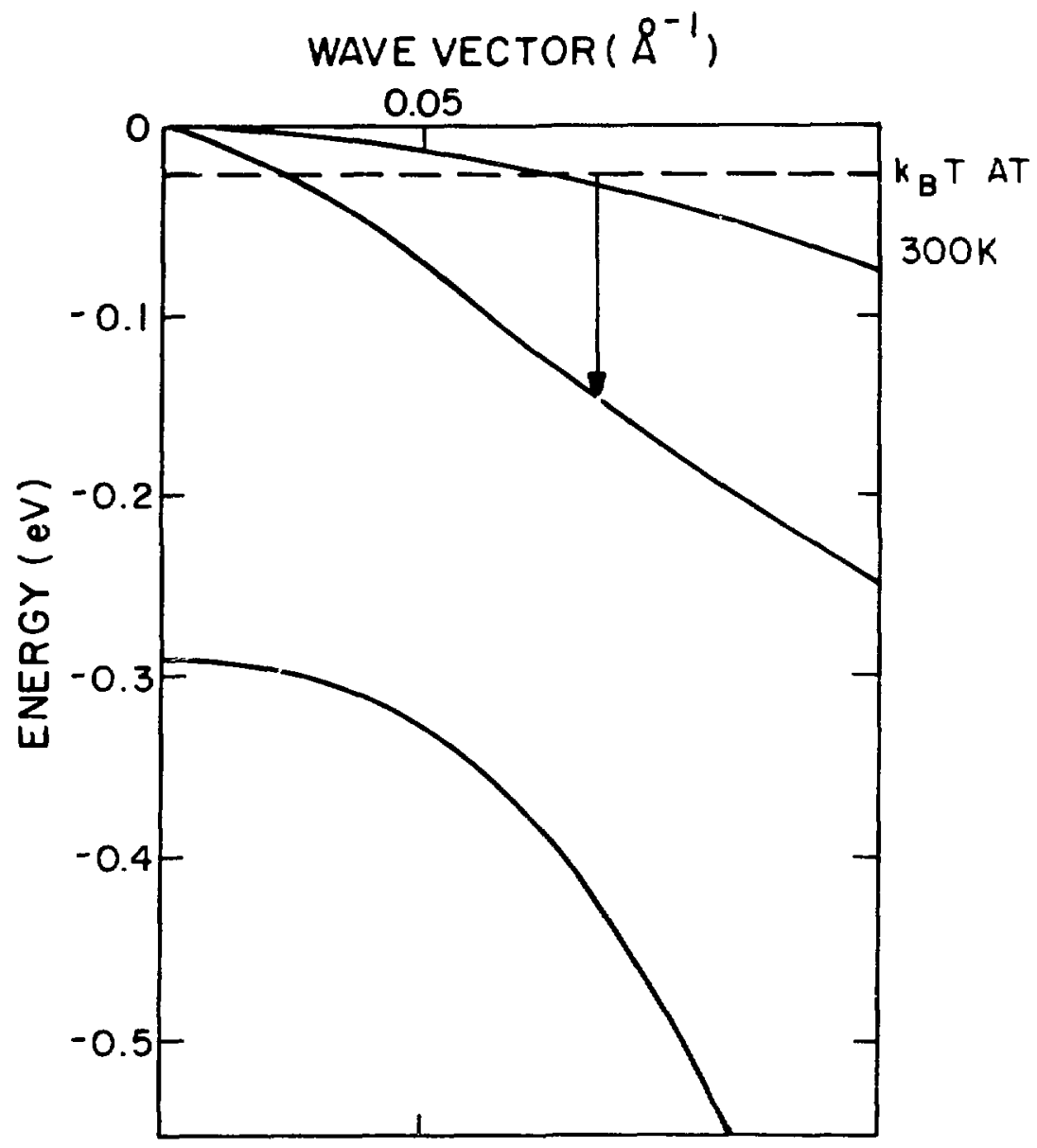

Figure $V-1$. Valence band structure of Ge, after keilmann (Ref. 1). The arrow shows the hole transition induced by 10.6 $\mathrm{\mu m}$ 1ight. Note that transitions can occur between these bands over a large range in optical photon energies. 
absorption associated with this optical transition can be bleached. Several things in the structure of Ge conspire to make this absorption extremely broadband and uniform (see Fig. 2). Basically, the increase in dipole moment with increasing wave vector is balanced by the decrease in the population of holes and by the density of states in the light hole band. The scattering of the light holes by acoustic phonons occurs on a subpicosecond time scale and the system completely relaxes in a matter of picoseconds by the emission of several phonons. 4

The model that has been commonly used ${ }^{4,5}$ to describe the saturable absorption of p-type $G e$ is essentially that for an infinitely broadband saturable absorber given in Chapter IV, section B. This model is an approximation, as is apparent from Fig. 2, which shows a variation of absorption with wavelength. Nevertheless, it has been successful in describing the saturation characteristics of Ge with only minor modification. One of the modifications has been the use of a frequency-dependent saturation intensity. ${ }^{5}$ The first attempt to model the dependence of the saturation intensity on wavelength used a model based purely on the relaxation process mentioned above. 6 This model predicts that there should be a factor of two decrease in saturation intensity as the wavelength goes from $10.6 \mu \mathrm{m}$ to $9.6 \mu \mathrm{m}$. Just the opposite behavior is 


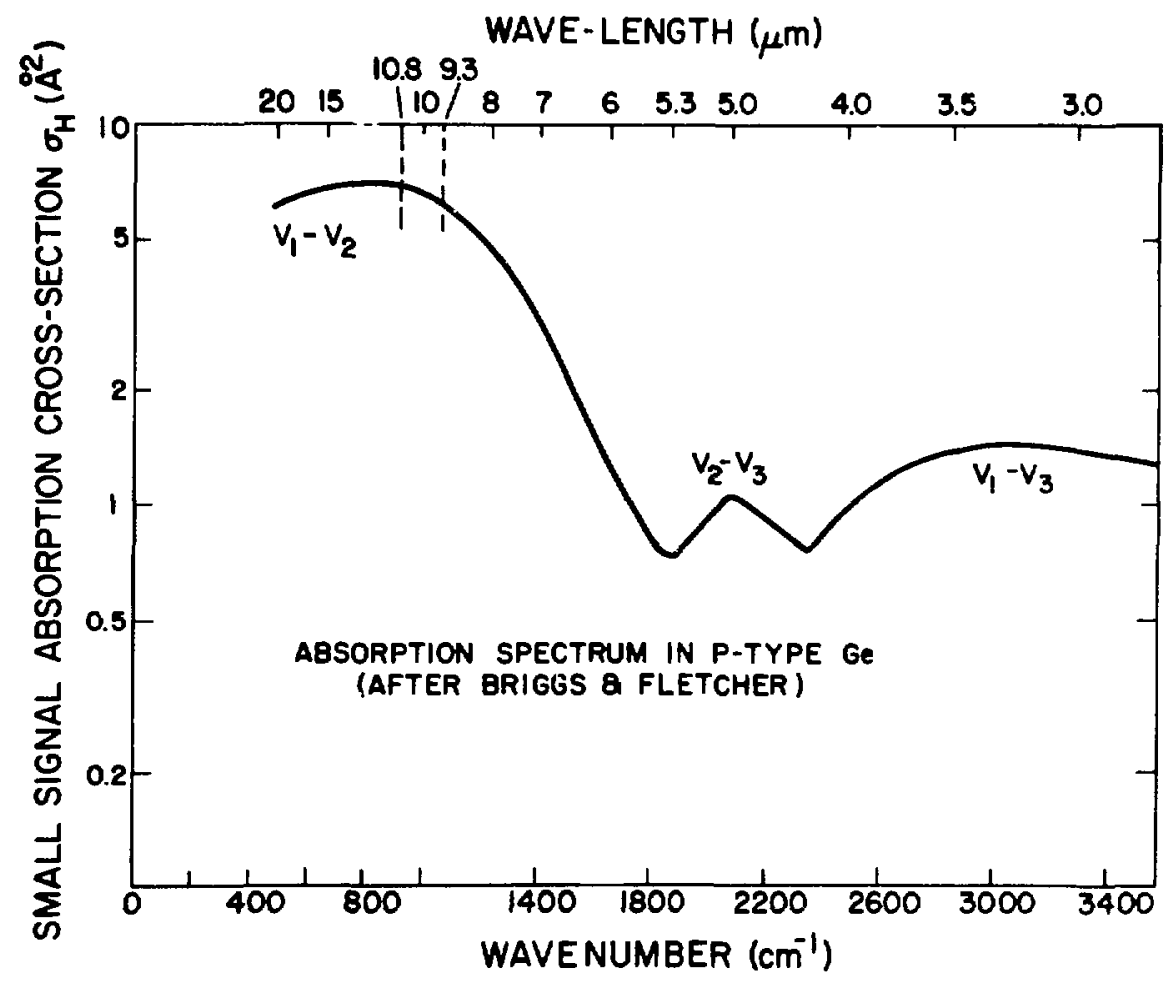

Figure $V-2$. Absorption cross-section as a function of wave number in p-type Ge (after Ref. 2). The absorption from the heavy to the light hole band is labeled $v_{1}-V_{2}$. other features are due to transitions between other combinations of bands. The $\mathrm{CO}_{2}$ laser spectrum falls between 9.3 and $10.8 \mu \mathrm{m}$. Over this range, there is only a $20 \%$ variation in cross-section. The width of the absorption band is on the order of the laser frequency. 
observed experimentally. Since this very simple model was published, a very careful analys is has been done by Ralph James. ${ }^{7}$ He uses degenerate $\vec{k} \cdot \vec{p}$ perturbation theory and a model of Ge which realistically accounts for the anisotropic and nonparabolic valence band structure. This model agrees with the simple analytic formula for the absorption coefficient used here and by others. His model does not supply another analytic form for the absorption coefficient. James also successfully predicts the observed dependence of saturation intensity on wavelength.

The most complete analytic and experimental characterization of the absorption that occurs in p-type Ge has been done by claude Phipps. ${ }^{8}$ In this model, even the Drude-zener absorption of the light holes is included (that for the heavy holes is miniscule and can be ignored). This essentially modifies the total absorption by making the residual nonsaturable absorption and the small signal saturable absorption coefficients dependent upon doping density.

That is, the total local absorption coefficient as a function of intensity can be determined by first partitioning the population of holes, $N$, between the heavy and light hole densities by

$$
N_{L}=(N / 2)\left[1-\left(1+|E|^{2} / E_{S}^{2}\right)^{-\frac{1}{2}}\right]
$$


and

(1b) $\quad N_{H}=(N / 2)\left[1+\left(1+|E|^{2} / E_{S}^{2}\right)^{-\frac{1}{2}}\right]$

where $E_{S}$ is the saturation field. Then the total absorption coefficient is

$$
\alpha_{\text {tot }}=\alpha_{r}+N_{L} \sigma_{L} / 2+\left(N_{H}-N_{L}\right) \sigma_{12} / 2
$$

$$
=\alpha_{r}^{\prime}+\alpha_{0}\left(1+|E|^{2} / E_{s}^{2}\right)^{-\frac{1}{2}}
$$

where $\alpha_{r}{ }^{\prime}=\alpha_{r}+N \sigma_{L} / 4$ and $\alpha_{0}=N\left(\sigma_{12} / 2-\sigma_{L} / 4\right)$.

The absorption cross sections are well known, as is the residual absorption coefficieit. I take $3,9 a_{r}=$ $0.017 \mathrm{~cm}^{-1}, \sigma_{12}=6.8 \AA^{2}$, and $\left(\sigma_{L} / \sigma_{12}\right)=0.085$.

The success of this model for the absorption coefficient is shown in Fig. 3.8 Here two models of the absorption are compared to experimental data, and it is shown that by including the Drude-zener absorpton of the light holes, a better fit to the experimental data is obtained.

B. Model of DFWM for p-type Ge

A model for DFWM in p-type Ge is obtained by deriving an expression for the polarizability from our knowledge of the saturation behavior and the inherent properties of 


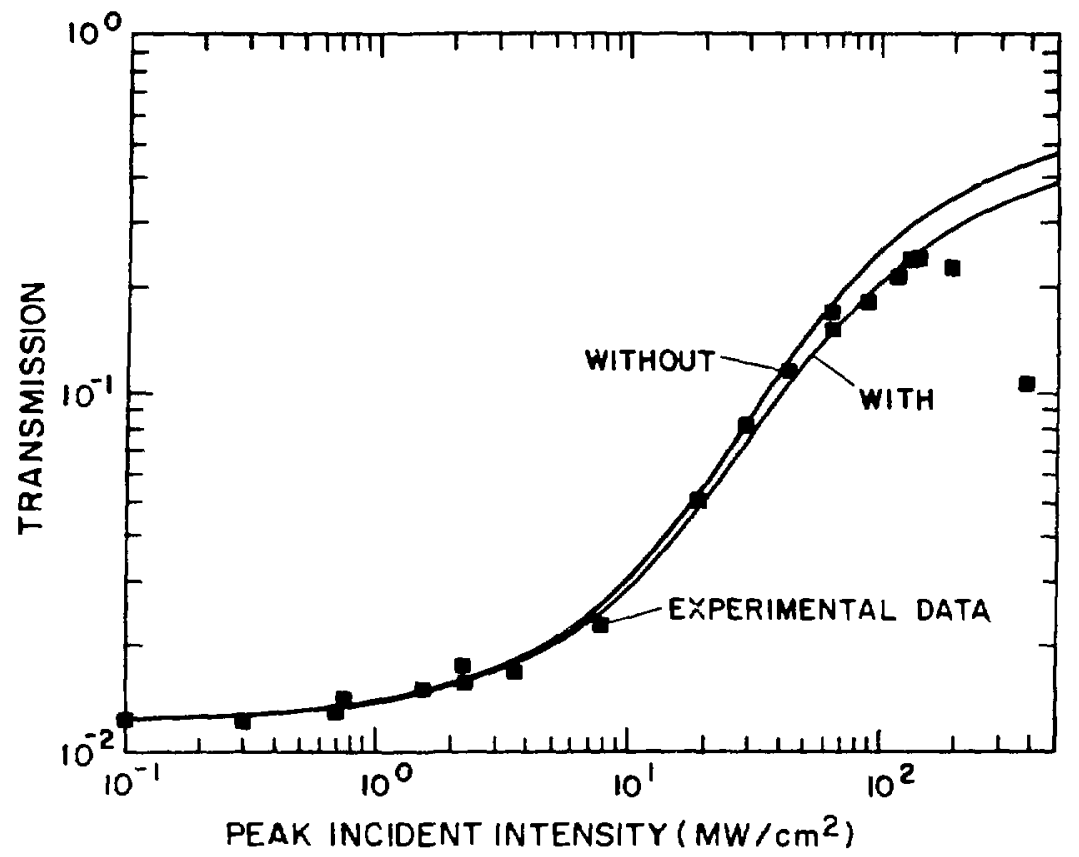

Figure $V-3$. The effect of including Drude-zener absorption in modeling saturable absorption in $p$-type Ge, after Phipps (see Ref. 8). The data and models are for uniform spatial and temporal illumination. 
Ge. This polarizability is expanded to firct order in the weak probe fields. Then the solutions of the previous chapter can be appiied.

The polarizability derived here has two major components. The first component is derived from the previous section, and models the saturation behavior of $p$-type Ge. The second component is derived from the inherent third-order nonlinear susceptibility, as discussed in Chapter III. The linear index of refraction is not incorporated as an independent entity in the polarizability, but appears as an additional factor of $n$ in various coefficients of the polarizability and the wave equation. The polarizability is taken as

(3) $P=\frac{i n \alpha_{r}}{2 \pi k_{0}} E+\frac{i n \alpha_{0}}{2 \pi k_{0}}-\frac{E}{\left(1+|E|^{2} / E_{s}^{2}\right)^{\frac{3}{2}}}+3 \times 3|E|^{2} E$

where $\alpha_{r}$ and $\alpha_{0}$ are defined in the previous section and $x_{3}$ is an effective third-order nonlinear susceptibility as in chapter III. The component of $P$ that describes the saturable absorption is of the same form as the broadband inhomogeneous saturable absorber treated in section B of Chapter IV. The third order nonlinear susceptibility has been treated in chapter III. The combination of these in Eq. 3 is basically treated by combining the two previous treatments. 
The justification for this polarizability is 1) that it models the inherent nonlinearity in the same way as a previous model for intrisic Ge, where it has been successiul. 2) It successfully models saturable absorption in p-type Ge. And 3 ) it is a good analytic approximation to the more complete model developed by James, which has no other analytic form. The analytic form is necessary because I wish to expand the polarizability in a perturbation series in the weak probe fields.

In ihis case, the expansion has been done in the previous chapters. Combining these results I obtain the propagation equations

$$
\begin{aligned}
& \frac{d E_{f}}{d z}=\left[-\alpha_{p R}+i \beta\left(\rho_{f}{ }^{2}+2 \rho_{b}{ }^{2}\right)\right] E_{f}, \\
& \frac{d E_{b}}{d z}=\left[\alpha_{p R}-i \beta\left(\rho_{b}{ }^{2}+2 \rho_{f}^{2}\right)\right] E_{b},
\end{aligned}
$$

$$
\frac{d E_{1}}{d z}=\alpha E_{1}+i k * E_{2} * \exp \left(i \phi_{+}\right)
$$

and

$$
\frac{d E_{2}}{d z}=-\alpha E_{2}-i \kappa * E_{1} * \exp \left(i \phi_{+}\right),
$$

where $\rho_{j}=\left|E_{j}\right|$. Equations for $\rho_{f}, \rho_{b}$ and the sum of the pump beam phases are obtained from Eqs. 5. 
These are

(6a) $\frac{d \rho_{f}}{d z}=-\alpha_{p R} \rho_{f}$,

(6b) $\frac{d \rho_{b}}{d z}=\alpha_{p R} \rho_{b}$,

and

(7)

$$
\frac{d \phi+}{d z}=\beta\left(\rho_{b}^{2}-\rho_{f}^{2}\right) .
$$

The coupling coefficients are given by

(8)

$$
\alpha_{p R}=\frac{\alpha_{\dot{r}}}{\cos (\theta)}+\frac{\alpha_{0}}{\pi \cos (\theta)\left[1+\left(\rho_{f}+\rho_{b}\right)^{2}\right]^{\frac{1}{2}}} k(b),
$$

(9a)

$$
\begin{aligned}
\alpha=-\frac{\alpha_{r}}{\cos (\theta)}- & -\frac{\alpha_{o}}{\pi \cos (\theta)\left[1+\left(\rho_{f}+\rho_{b}\right)^{2}\right]^{\frac{1}{2}}} \times \\
& \left\{k(b)+\frac{E(b)}{1+\left(\rho_{f}-\rho_{b}\right)^{2}}\right\} \\
& +2 i \beta\left(\rho_{f}{ }^{2}+\rho_{b}{ }^{2}\right),
\end{aligned}
$$

and

(9b)

$$
\begin{aligned}
& K= \frac{i \alpha_{o}}{\cos (\theta)\left[1+\left(\rho_{f}+\rho_{b}\right)^{2}\right]^{\frac{1}{2}}} x \\
&\left\{\frac{\rho_{f}^{2}+\rho_{b}^{2}}{2 \rho_{f} \rho_{b}}[K(b)-E(b)]-\frac{\left(\rho_{f}-\rho_{b}\right)^{2}}{1+\left(\rho_{f}-\rho_{b}\right)^{2}} E(b)\right\} \\
&+2 i \beta \rho_{f} \rho_{b} .
\end{aligned}
$$


The coefficient $\beta$ is given by

$$
\beta=\frac{6 \pi k_{0} x_{3} E_{s}{ }^{2}}{n \cos (\theta)}
$$

as in Chapter III, except that now $\beta$ is normalized to the saturation field.

Equation 7 for $\phi_{+}$shows that this quantity is not a constant in this case. The numerical model developed in Chapter IV still covers this case. The variation with distance of $\phi_{+}$follows the same dependence as in the case of intrinsic Ge, except that the condition $\rho_{f}=\rho_{b}$ cannot be met at all points in the interaction length, since the counterpropagating pump beams are attenuated by the medium. Thus, there is necessarily some phase mismatch introduced by the propagation. This is most important when the medium is not totally bleached, while the intensity is such that the inherent third-order nonlinear susceptibility plays a major role in the mixing process.

C. DFWM experiments in $p$-type Ge

In this section, I present results of DFWM experiments in p-type Ge. Results for three samples with small-signal intensity transmissions of $70 \%, 43 \%$, and $8 \%$ are discussed. These samples are chosen to provide a range in small- 
signal saturable absorption coefficient, and hence coupling, of $\alpha_{0} L=0.36$ to 2.5 .

Figure 4 shows the reflectivity versus pump intensity for the $70 \%$ transmitting sample. This $6-\mathrm{mm}$ sample is interesting because each of the three known mechanisms for DFWM in p-type Ge are observed distinctly. In the intensity regime below $10 \mathrm{MW} / \mathrm{cm}^{2}$, the resonantiy enhanced contribution of the saturable absorption is observed. A reflectivity 40 times greater than the reflectivity obtained in intrinsic Ge is observed at a pump intensity of $1 \mathrm{MW} / \mathrm{cm}^{2}$ in this sample, which has a doping density of on $1 \mathrm{y} N=7.7 \times 10^{14} / \mathrm{cm}^{3}$. In the region from 10 to $100 \mathrm{MW} / \mathrm{cm}^{2}$, the inherent nonlinearity of Ge becomes dominant, and a dependence approaching I? is observed for the reflectivity. Finally, above 100 $\mathrm{MW} / \mathrm{cm}^{2}$, the wave mixing is dominated by the free-carrier generation process. The largest reflectivities I have observed are presented in this plot.

The curve in the figure is the model of the previous section. This model uses $\alpha_{0}=0.272 \mathrm{~cm}^{-1}, \alpha_{r}{ }^{\prime}=$ $0.028 \mathrm{~cm}^{-1}$, and $x_{3}=3.5 \times 10^{-11}$ esu. The values for the absorption coefficients are set by the small-signal transmission and our knowledge of the saturation behavior of Ge. The only adjustable parameter for fitting the curve to the data is the effective third 


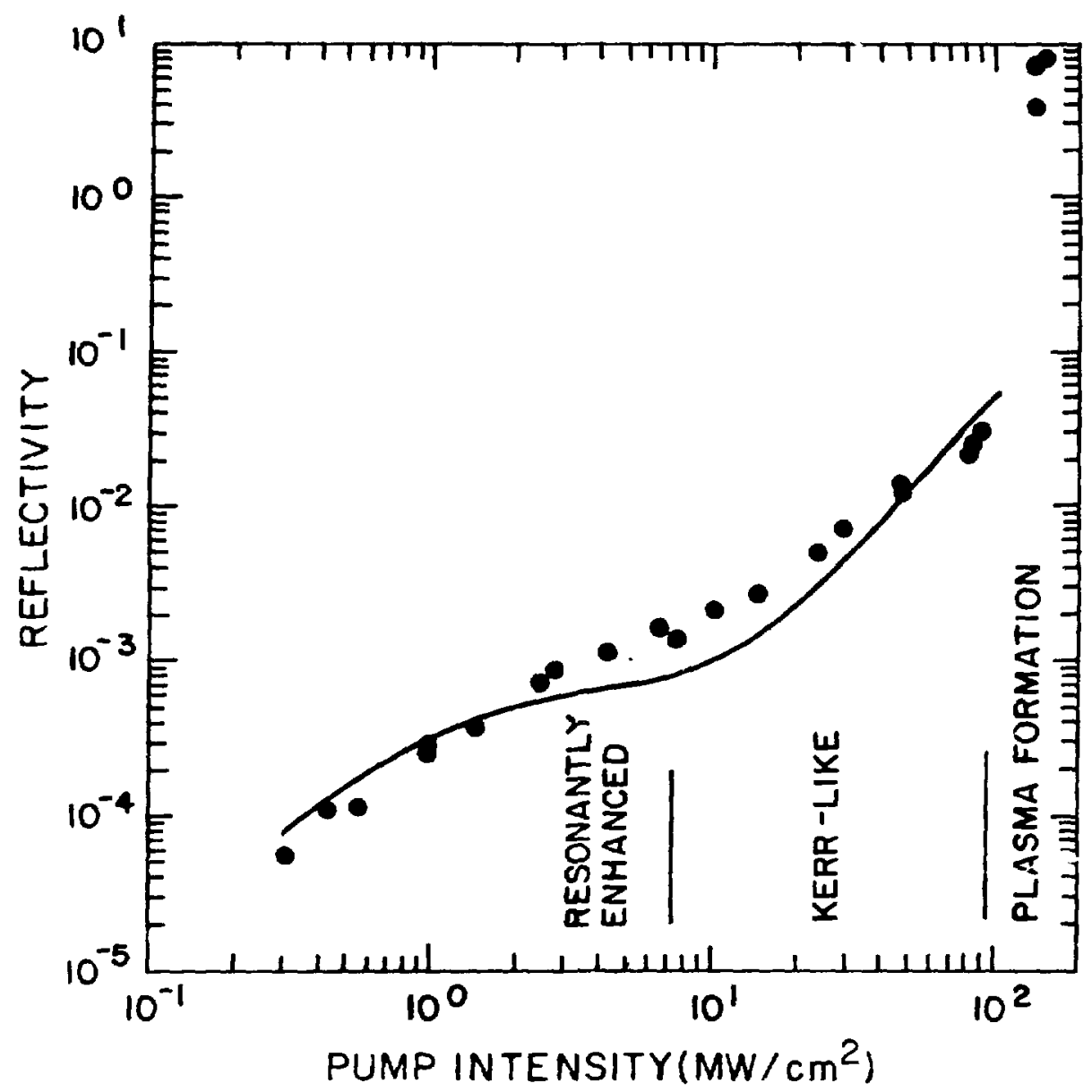

Figure $V-4$. DFWM at $10.6 \mu \mathrm{m}$ in a $6-\mathrm{mm}$, p-type sample of Ge. The small-signal transmission of the sample is $70 \%$. All three mechanisms are clearly distinguishable in this data. Below $10 \mathrm{MW} / \mathrm{cm}^{2}$, OFWM by saturable absorption dominates. Between 10 and $100 \mathrm{MW} / \mathrm{cm}^{2}$, the inherent nonlinear index of the bound electrons dominates. Above $100 \mathrm{MW} / \mathrm{cm}^{2}$, plasma formation is the dominant mechanism. The solid curve is my model based on the numerical solution of Eqs. 4-10. 
order nonlinear susceptibility, $x_{3}$, which must range between 2.5 to $4 \times 10^{-11}$ esu to be consistent with the results presented in Appendix $A$ and the results of Chapter III. This actually depends upon the crystal orientation, which is not known for the samples studied here.

The major effect of varying $x_{3}$ is to transiate the linear portion of the curve to the right or left, depending on whether the value of $x_{3}$ is decreased or increased, respectively. In this case, the value chosen for $x_{3}$ gives a good fit to the data and is consistent with previous results.

Results for a DFWM experiment in a 3-mm-long sample of p-type Ge with a small-signal transmission of $43 \%$ are shown in Fig. 5. For this sample, $N=3.6 \times 10^{15} / \mathrm{cm}^{3}$, $\alpha_{0}=1.337 \mathrm{~cm}^{-1}$ and $\alpha_{r}{ }^{\prime}=0.069 \mathrm{~cm}^{-1}$. The model uses $x_{3}=3 \times 10^{-11}$ esu. In this case, the resonant enhancement due to the saturable absorption process dominates below intensities of about $30 \mathrm{MW} / \mathrm{cm}^{2}$. In this regime, reflectivities 40 times that expected in intrinsic Ge are again observed. The model describes the trend in this regime well, but predicts twice the observed reflectivity. The data centered around the pump intensity of $75 \mathrm{MW} / \mathrm{cm}^{2}$ lie substantially above the model. However, this is at least partly explained by the onset of plasma formation. A single point showing a reflectivity 


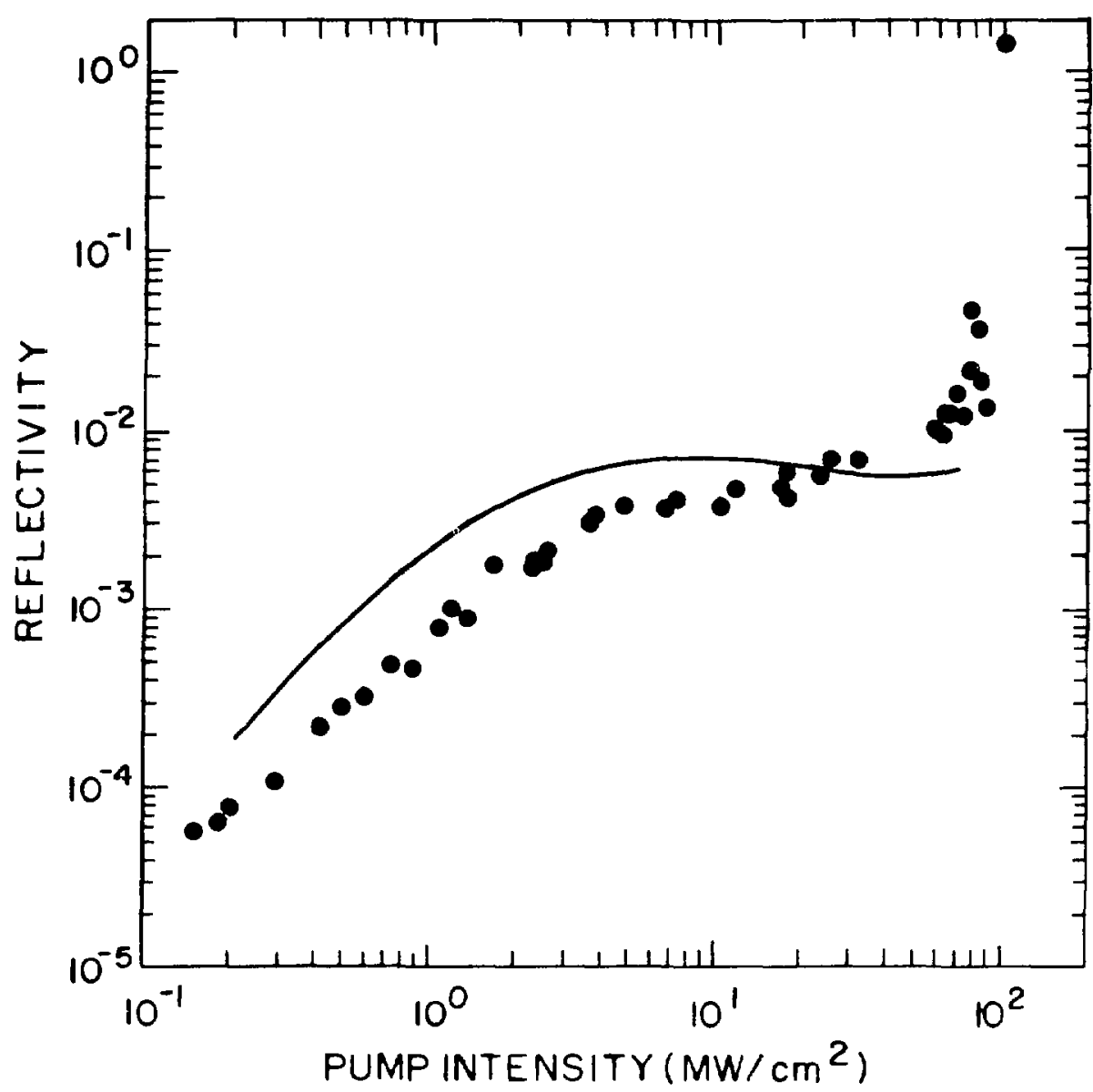

Figure V-5. DFWM in a 3-mm, p-type sample of Ge. The small-signal transmission in this sample is $43 \%$. The solid curve is the model presented in the text. This model follows the trend of the data below the plasma formation threshold of $80 \mathrm{MW} / \mathrm{cm}^{2}$. There is a factor-of-2 discrepancy between the model and the data. The only free parameter in the model is $x_{3}$, which still has a limited range. The only possible effect this parameter could have is to increase the prediction made by the model, not a decrease as is needed. 
of over $100 \%$ is seen at about $100 \mathrm{MW} / \mathrm{cm}^{2}$.

Results from the third sample are shown in Fig. 6 .

This sample is a 1-cm-long piece of Ge doped p-type to $N=3.2 \times 10^{15} / \mathrm{cm}^{3}$, with a small-signal transmission of $8.2 \%$. The curve shown in the plot is the numerical model with the appropriate values for the field absorption coefficients, $\alpha_{0}=1.186 \mathrm{~cm}^{-1}$ and $\alpha_{r}{ }^{\prime}=0.064 \mathrm{~cm}^{-1}$. A value of $x_{3}=3 \times 10^{-11}$ esu 15 assumed. Measured enhancements over the predicted effect in intrinsic Ge are about a factor of 10 . The data lie about a factor of 15 below the prediction of the model at a pump intensity of $1 \mathrm{MW} / \mathrm{cm}^{2}$. The change from DFWM via saturable absorption to DFWM via the inherent nonlinear index is predicted by the model. However, the data do not conform to the model, and no conclusion regarding this effect can be drawn.

Although the data in Fig. 6 show more scatter than in other experiments, there is no other reason to believe that the data are inaccurate. There appears to be a trend in all the data for the model to do worse as the absorption of the sample increases. Note, however, that the doping density for this sample is actually about $10 \%$ less than for the sample of Fig. 5, where the model did much better. Thus, it appears that the discrepancy is due to the total absorption and not to the doping density. 


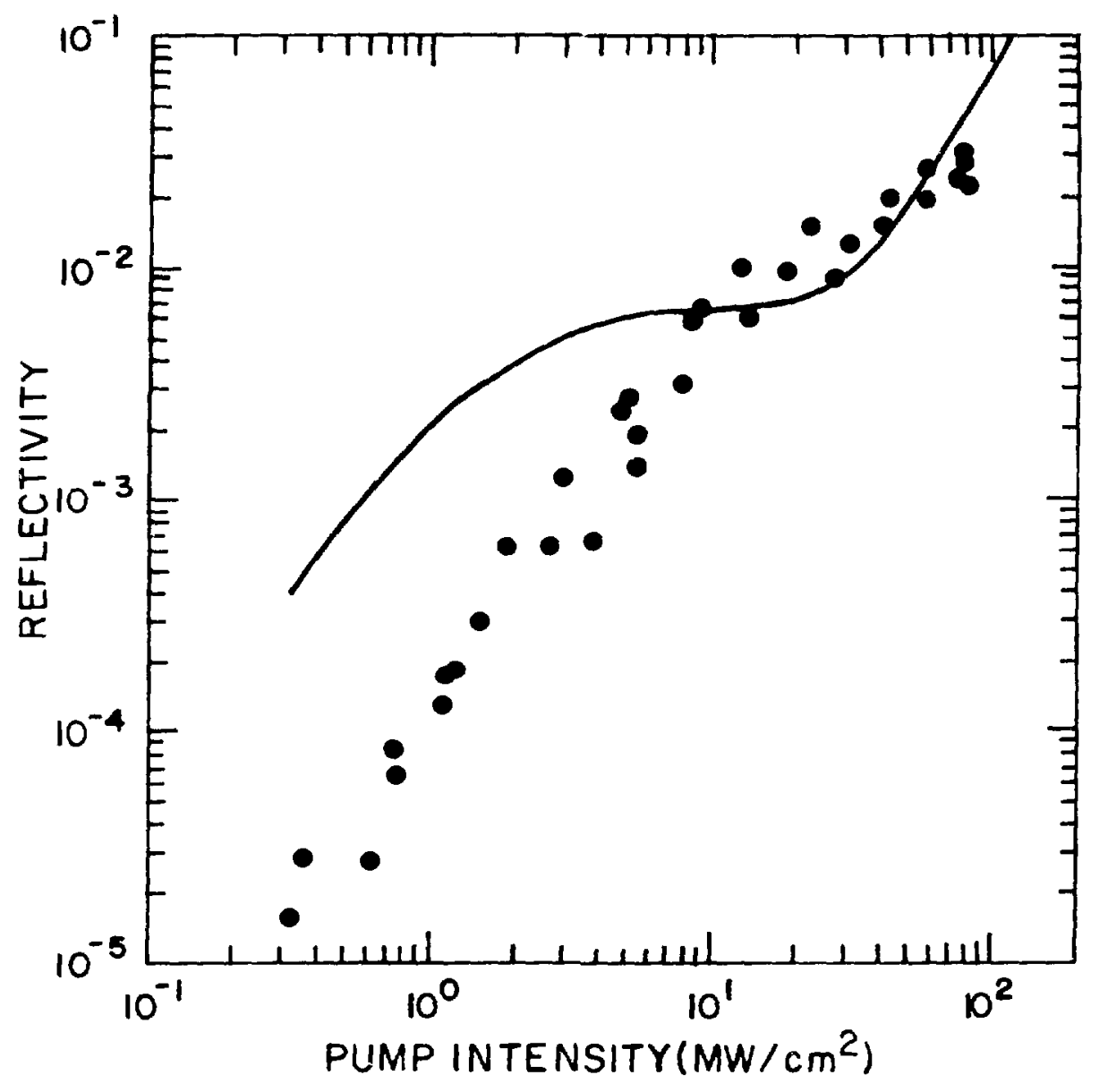

Figure V-6. DFWM in a $1-c m$, p-type sample of Ge. The small signal transmission of the sample is $8 \%$. The curve is the model presented in the text based on the numerical solution of Eqs. 4-70. 
Since absorption of all beams in the wave mixing process is taken into account, this result is perplexing.

\section{Discussion and conclusions}

DFWM has been modeled in p-type Ge by considering two nonlinear mechanisms: the saturable absorption due to p-type doping and the inherent third-order nonlinear susceptibility of Ge. This model works well for small field absorption coefficients (or intensity transmission greater than 40\%), but fails for larger absorption coefficients (samples with small-signal transmission less than $10 \%)$.

The model is based on a polarizability derived from our knowledge of the saturable absorption and the third-order nonlinearity of the bound electrons. The application of the absorptive part of the polarizability to describing saturable absorption in p-type Ge has been very successful. It is therefore believed that this part of the polarizability is accurate, even though it is only an approximation to the more complete model given by James. Although the contribution of the inherent nonlinearity due to bound electrons in Ge is also well understood, and this mechanism has been successful in describing DFWM in intrinsic Ge, it is not so clear that this is the only mechanism for induced index changes in p-type $G$ s 
In p-type Ge, four possible mechanisms for nonlinear index changes exist: first, there is the bound electron contribution, as discussed above and in chapter III. Next, there is a contribution predicted by James associated with the resonant transition which produces saturable absorption. ${ }^{7}$ Third, there is a temperature dependent change in index of refraction, ${ }^{10}$ which occurs because of heating by absorption of the laser beam. These three contributions are all positive. A fourth negative contribution is from the free-carrier intraband transitions. 11 This last contribution is most important when excess free carriers are generated in the plasma formation process. When the carrier concentration is constant, as it is here, this contribution is believed to be negligible.

I would like to stress that none of the last three contributions have been isolated and measured directly in p-type Ge. Indeed, the only one for which there is experimental evidence is the temperature-dependent change in index.

I will discuss the nonlinear index associated with the resonant transition and the heating effect here.

Two parts of the polarizability are associated with any optical transition. One part is in phase with the driving field, and gives a contribution to the index of 
refraction of the medium (or the real part of the susceptibility). The other part is $90^{\circ}$ out of phase with the driving field and contributes to the absorption (or imaginary part of the susceptibility.

I employ a simple model to derive that part of the polarizability due to the saturable absorption of p-type Ge. In this model, an ensemble of two-level absorbers, uniform in frequency distribution and strength, is assumed. 4 The nonlinear index contribution, or real part of the susceptibility, from such an ensemble vanishes, because for each contribution resulting from a subset of the ensemble to the blue of the optical frequency, there is a corresponding contribution of opposite sign from a subset of the ensemble to the red of the optical frequency. This model is capable of describing the saturable absorption because only those two-level systems with frequency near the optical frequency contribute to the absorption. The approximation is good in this case. But the nonlinear index contiputions can come from systems much further from resonance. The assumption of a uniform distribution may very well break down in attempting to calculate the nonlinear index effect of the resonant transition. From James' more realistic mode ${ }^{12}$ for the complex susceptibility of p-type Ge associated with the optical 
transition, a nonlinear index resulting from the resonant transition is predicted. He predicts that the magnitude of the nonlinear index associated with the iransition is equal to that of the inherent nonlinear index at a doping density of about $N=1 \times 10^{15} / \mathrm{cm}^{3}$. This is of the order of the densities of the samples I employ. The nonlinear index James predicts begins to saturate at about $10 \mathrm{MW} / \mathrm{cm}^{2}$. Since this nonlinear index has the same sign as the inherent nonlinear index, it should enhance the effects of the inherent nonlinear index. No evidence has been seen to support this.

The second effect that may be important $c$ an be estimated and compared to the inherent nonlinear index. This is the temperature dependent change in index of refraction. A measurement of the change in index of refraction with temperature has produced the value 10 $\mathrm{dn} / \mathrm{dT}=4 \times 10^{-4} /{ }^{\circ} \mathrm{K}$. The temperature increase due to absorption of the laser energy can be calculated from the specific neat, ${ }^{13} \mathrm{c}=0.3222 \mathrm{~J} / \mathrm{g}^{\circ} \mathrm{K}$, the density, 14 $\rho_{G e}=5.3243 \mathrm{~g} / \mathrm{cm}^{3}$, and the intensity absorption coefficient, 8

$$
\alpha_{I}=2 \alpha_{r^{\prime}}+\frac{2 \alpha_{0}}{\sqrt{1+I / I_{S}}} .
$$


The local change in temperature is given by

$$
\Delta T=\frac{\alpha_{I} I T}{C \rho}
$$

where $\tau$ is the laser pulse duration, here taken as 1 ns. The change in index of refraction due to this effect is given by $\Delta n=(d n / d T) \Delta T$. The magnitude of this change in index of refraction is plotted versus intensity in Fig. 7, for a doping density of $\mathrm{N}=1.3 \times 10^{15} \mathrm{~cm}^{-3}$. The change in temperature for the data in this figure ranges from $6 \times 10^{-5}$ to $10^{-2}{ }^{\circ} \mathrm{K}$. Also plotted in the figure is the change in index due to the inherent, bound electron contribution to the nonlinear index, where I have taken $x_{3}=2.5 \times 10^{-11}$ esu, and values for the nonlinear index change associated with the resonant transition taken from James work, using the same dopant density as above ${ }^{12}$. A mild saturation of the temperature dependent effect can be seen, which is due to the saturation of the absorption. Both the effect predicted by James and the temperature effect scale linearly with the doping density. Thus, they are both negligible in intrinsic Ge. In p-type Ge, they can easily be the same order of magnitude as the bound electron contribution. 


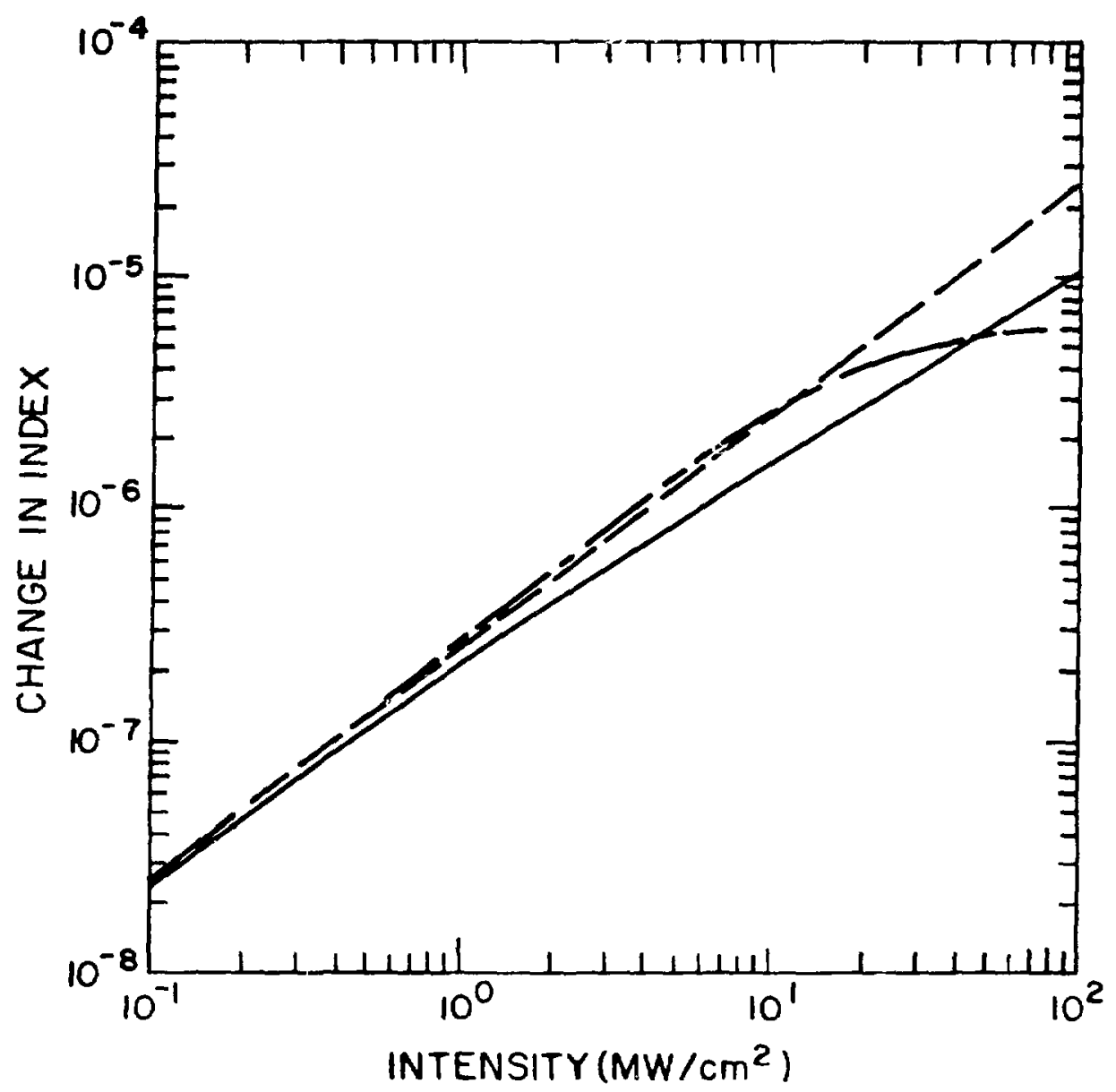

Figure V-7. Comparison of the change in index due to thermal changes (solid curve), due to the bound electron contribution (dashed straight line), and due to the interband transition (dashed curve). The thermal contribution and the interband contribution depend linearly on the concentration of holes. In this case $N=1.3 \times 10^{15} \mathrm{~cm}^{-2}$ and the laser pulse duration is assumed to be $1 \mathrm{~ns}$. The thermal contribution is flux dependent, not intensity dependent. By varying the 1 aser pulse duration and the dopant concentration, any of the three mechanisms may be made to dominate. 
A crucial difference between the temperature dependent effect and the other two effects is that the temperature effect depends on the flux of a 1-ns pulse, and not on the intensity as the others do. This is because the heating for such a short pulse depends only on the energy deposited in a certain volume, and diffusion of the heat can be ignored, at least for laser beams of the diameter and duration $I$ use. Thus, if the flux remains constant, but the duration of the pulse is increased, the effect of the temperature dependent change in index of refraction will increase relative to the other contributions.

In DFWM by such a mechanism, the grating produced by the heating effect will remain until the heat can diffuse away.

In any event, the contribution of nonlinear index effects adds in quadrature to the absorptive effects in the simplest of models for DFWM (where phase mismatch effects are ignored). Thus, neither of these effects can account for the discrepancy between the data and the model in $\mathrm{Fig} .6$.

In conclusion, DFWM in p-type Ge has been modeled assuming two mechanisms contribute to the nonlinear wave mixing process; saturable absorption of a resonant transition between the heavy- and light-hole bands and the inherent nonlinear susceptibility of Ge. Three other 
possible mechanisms for nonlinear refraction of light in p-type Ge have been discussed. No mechanism has been identified that can account for the discrepancy between experimental data and the model for large absorption.

of the three possible mechanisms for nonlinear refraction mentioned here, none have been observed using short laser pulses. Only the temperature dependent effect has ever been observed experimentally.

The agreement between the observed OFWM in p-type Ge and the model presented in Section $B$ is good for the samples of Figs. 4 and 5 . The data of Fig. 4 clearly show the three mechanisms for DFWM in $p$-type Ge. In the data of Fig. 5, only the resonantly enhanced absorption effect and the free-carrier generation effect are clearly distinguished. 


\section{Footnotes}

1. F. Keilmann and Jurgen Kuhl, "Broadband modulation of

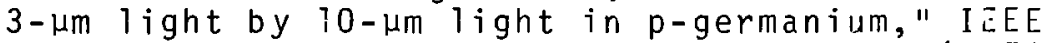
Journal of Quantum Electronics QE-14, 203 (1978).

2. A. H. Kahn, "Theory of infrared absorption of carriers in germanium and silicon," Physical Review 97, 1647 $(1955)$.

3. H. B. Briggs and R. C. Fletcher, "Absorption of infrared light by free carriers in germanium," Physical Review 91, 1342 (1953).

4. F. Keilmann, "Infrared saturation spectroscopy in p-type germanium," IEEE Journal of Quantum Electronics QE-12, 592 (1976).

5. C. R. Phipps, Jr. and S. J. Thomas, "Saturation behavior of p-type germanium at $\mathrm{CO}_{2}$ laser wavelengths," Optics Letters 1, 93 (1977).

6. M. Sargent III, "Relaxation of hot holes in $p-G e, "$ Optics Communications 20, 298 (1977).

7. R. B. Jàmes and D. L. Smith, "Theory of nonlinear infrared absorption in p-type germanium, "Physical Review Letters 42, 1495 (1979).

8. C. R. Phipps, Jr., unpublished. Some of the analysis done by Phipps is also presented in a paper by D. E. Watkins, C. R. Phipps, Jr., and S. J. Thomas, "Observation of amplified reflection through degenerate four-wavemixing at $\mathrm{C}_{2}$ laser waveiengths in germanium," Optics Letters $\underline{6}, 76$ (1981).

9. E. D. Capron and 0. L. Brill, "Absorption coefficient as a function of resistance for optical germanium at $10.6 \mathrm{\mu m}, "$ Applied Optics 12, 569 (1973).

10. The best source for the temperature dependent change in index of refraction at $10.6 \mu \mathrm{m}$ is the manufacturers data given by $N$. V. Metallurgie Hoboken-overpelt S. A. They give the value used here. A value at $5 \mu \mathrm{m}$ is given by M. Cardona, W. Paul, and H. Brooks, "Dielectric constant of germanium and silicon as a function of volume," Journal of the Physics and Chemistry of Solids 8,204 (1959). 
11. R. James, private communication. This contribution is due to nonlinearities in the free-carrier intraband contribution to the index of refraction. It is not the same as the nonlinear index produced by the generation of free carriers, as in Chapter VI. In Chapter VI, the change in index of refraction is due to an intensity dependent change in the number of free carriers. The response of individual electrons is a linear contribution to the index of refraction, the nonlinearity arises purely from the generation of carriers.

12. R. B. James and D. L. Smith, "Laser-induced changes in the dispersive properties of $p-G e$ due to intervalence-band transitions," Physical Review B 23 , 4044 (1981).

13. P. Flubacher, A. J. Leadbetter, and J. A. Morrison, "The heat capacity of pure silicon and germanium and properties of their vibrational frequency spectrum," Philosophical Magazine 4,273 (1959).

14. Gmelins Handbook of Inorganic Chemistry, 8 th Edition, "Germanium," Weinheim, Verlag Chemie, GmbH 1958. 


\section{CHAPTER VI}

DFWM IN THE PLASMA FORMATION REGIME OF GE

\section{Introduction}

In the course of a DFWM experiment in p-type Ge, I have unexpectedly observed a rapid and dramatic increase in the wave mixing process over a small range of intensity near the surface damage threshold. Since this initial observation, additional experimental work has lead to the identification of the DFWM mechanism with the nonlinear optical generation of free carriers. A model has been developed which successfully describes the order of magnitude of the wave mixing process and its dependence on pump intensity.

DFWM by optically induced free carriers in semiconductors has been studied by several authors. Jain, and earlier Woerdman, ${ }^{2}$ report resonantly enhanced DFWM at $1.06 \mu \mathrm{m}$ near the band gap of silicon. Both Kahn ${ }^{3}$ and $\mathrm{Jain}^{4}$ have reported similar observations at $10 \mu \mathrm{m}$ in $\mathrm{Hg}_{7-x} \mathrm{Cd}_{x} \mathrm{Te}$. In this material the band gap can be tuned to resonance by adjusting the stoichiometry $(x)$. Miller ${ }^{5}$ was the first to report extremely large nonlinearities in wave mixing experiments near the band gap of InSb. Most recentiy, Haueisen ${ }^{6}$ has observed resonantly enhanced three-wave mixing ! or forward phase 
conjugation) in InAs, employing the same type of nonlinearity. All of these studies have been near the band gap.

Jain ${ }^{7}$ has modeled the wave mixing in semiconductors. He notes that in these materials a large concentration of free carriers is generated by single-photon absorptica. This free-carrier plasma exhibits the spatial variations of the interfering probe and pump beams. This perturbs the index of refraction in the medium, which, in turn, creates the gratings that scatter the pump beams to generate the reflected beam. In the work presented here, the mechanism for scattering is taken to be the same as Jain's, but since $10.6 \mu \mathrm{m}$ is far from resonance with the band gap of Ge, the process for the formation of the optically induced free carriers is multiphoton.

In this chapter, I present the results of experiments in DFWM in Ge in the plasma formation regime and discuss the modeling of these results. The chapter is organized as follows: In section $A$, I review the process of $27 e e-c a r r i e r$ generation in Ge by $10 \mu \mathrm{m}$ light. In section $B$, I present experimental results and discuss a model for DFWM in this regime. Section $C$ deals with an experiment to observe the decay of an optically induced free-carrier grating in Ge by diffusion of the free carriers. A conclusion is given in section $D$. 
A. The plasma formation process in Germanium

The plasma formation process has been the subject of intensive study by Phipps. ${ }^{8}$ Experimental studies have also been pursued by Yuen ${ }^{9}$ and Danileiko. ${ }^{10}$ More recently, a theoretical model of the process has been proposed by James. 11 The formation of free carriers in Ge by $10.6 \mu \mathrm{m}$ light is believed to be due to a cascade process. In this process, free electrons in the conduction band (and free holes in the valence band, although James claims the electrons dominate ${ }^{11}$ ) absorb energy from the incident radiation. This energy is transferred to valence electrons through collisions. Some valence electrons gain enough energy in this process to cross the band gap. This model explains the $I^{5.5}$ intensity dependence observed for the process (see Fig. 1) and the indirect transition across the band made by the excited electrons. If the process were multiphoton absorption by individual electrons, the intensity dependence would be integral, since fractions of photons cannot be absorbed. The observed $I^{5.5}$ dependence indicates an indirect transition, since the indirect band gap is equivalent to 5.6 photons at a wavelength of $10.6 \mu \mathrm{m}$, and the direct gap is equivalent to 6.9 photons. The change in wave vector necessary for the indirect transition can come from the collisions responsible for the excitation. 
A careful experimental determination of the peak free-carrier density as a function of peak input intensity has been made by Phipps. ${ }^{12}$ Because of the relevance of this work to the modeling of DFWM in this regime, I present a discussion of the work here.

The free-carrier density is determined experimentally by carefully time-resolving the incident and transmitted signals of a single intense optical pulse above the plasma threshold in Ge. The free-carrier density is deduced from this measurement by use of a detailed model which relates the density to the instantaneous, intensity-dependent transmission.

When a laser induced plasma is generated at $\mathrm{CO}_{2}$ laser wavelengths in Ge, three types of carrier are produced. Each of these carriers effects the absorption in a different way. Three widely disparate time scales are involved in the evolution of their densities. The longest time scale is that for recombination, ${ }^{13}$ which is $10^{3}$ to $10^{6}$ times longer than the laser signal duration. This situation ensures that charge neutrality governs the densities of new holes and electrons created.

The next shorter time scale is that for the evolution of the plasma-controlled transmission. This is observed to be slightly faster than the laser pulse duration, that is about $300 \mathrm{ps}$. Much faster yet (about $z \mathrm{ps}$ ) is the time 
required to partition the local total density of holes between the heavy-and light-hole bands. ${ }^{14}$ The primary effect of this redistribution is bleaching of the associated interband transition that is responsible for most of the absorption of $10.6 \mu \mathrm{m}$. This bleaching is exactly analogous to that of p-type Ge, as discussed in the previous chapter. Thus, the absorption of the Ge in the plasma formation regime can be modeled analogously to that of $p$-type Ge, with one additional term from the Drude-zener effect of the electrons.

The analysis I present here is valid for intrinsic Ge. Only slight modifications are necessary to apply the results to either $p$-type or n-type Ge, and this analysis is sufficient for my needs. As in Chapter $V$, I first partition the total density of holes, $N$, between the heavy- and light-hole bands:

(1a) $\quad N_{L}=(N / 2)\left[1-\left(1+|E|^{2} / E_{S}^{2}\right)^{-\frac{1}{2}}\right]$

and

$$
N_{H}=(N / 2)\left[1+\left(1+|E|^{2} / E_{S}^{2}\right)^{-\frac{1}{2}}\right] .
$$

In the full treatment, $N$ includes both the holes induced by the generation of the free carrier plasma, and holes present in the medium either due to thermal excitation or p-type doping. In the present analysis, I assume intrinsic Ge, and ignore the negligible thermal contribution. 
Ignoring only the Drude-Zener component of the absorption due to the free heavy holes (it is extremely sma 11), the total absorption coefficient is now written

$$
\alpha_{\text {tot }}=\alpha_{r}+N \sigma_{e} / 2+N_{L} \sigma_{L} / 2+\left(N_{H}-N_{L}\right) \sigma_{12} / 2
$$

as in Eq. 2 of the previous chapter, but now including an absorption term due to the free electrons. The relative cross section for the free electron absorption is 15 $\sigma_{e} / \sigma_{12}=0.044$. The density of electrons is equal to the total density of holes by charge conservation. To deduce the free-carrier density from time resolved measurements of the optical transmission of Ge in the plasma formation regime, a reverse iterative procedure is used. First, the total density of free-carriers is estimated. This density is assumed to be constant throughout the entire length of the Ge sample. Equations 1 and 2 are then used to determine the local absorption coefficients as a function of intensity as the $10.6 \mu \mathrm{m}$ beam propagates through the medium. This allows a determination of the intensity as a function of distance through the medium, and hence the transmission. The transmission calculated in this way is compared to the measured transmission. If these agree, then the estimate for the density is good. If they don't agree, iteration can be used to improve the estimate of the density. 
Figure 1 shows the results of this process when applied to samples of Ge of three different lengths. In the figure, the threshold for strong plasma formation is seen to be about $200 \mathrm{MW} / \mathrm{cm}^{2}$. Above this intensity, excess carrier density varies with intensity to the 5.5 power. The agreement between three samples of widely differing length justifies the assumption that the density is constant along the length of the medium.

In the next section, I show how the density versus intensity profile is used to estimate the reflectivity for sufficiently thin samples of Ge.

B. DFWM in the plasma formation regime of Ge

The experimental technique for measuring reflectivity is described in chapter II. For the experiments discussed here, the laser is tuned to $10.59 \mu \mathrm{m}$ ( $\mathrm{P}-20$ of the $\mathrm{CO}_{2}$ laser) and the maximum intensity available in each pump beam is $150 \mathrm{MW} / \mathrm{cm}^{2}$. The intensity available in the counterpropagating pump beams is above the surface damage threshold for the antireflection-coated $G e$ in this geometry. The external angle between the probe and the pump beams is about $15^{\circ}$, corresponding to $4^{\circ}$ inside the medium. The samples studied are between 3 and $20-\mathrm{mm} 10 \mathrm{ng}$, ranging from optical grade (slightly n-type) to p-type, and either single-crystalline or polycrystalline. 


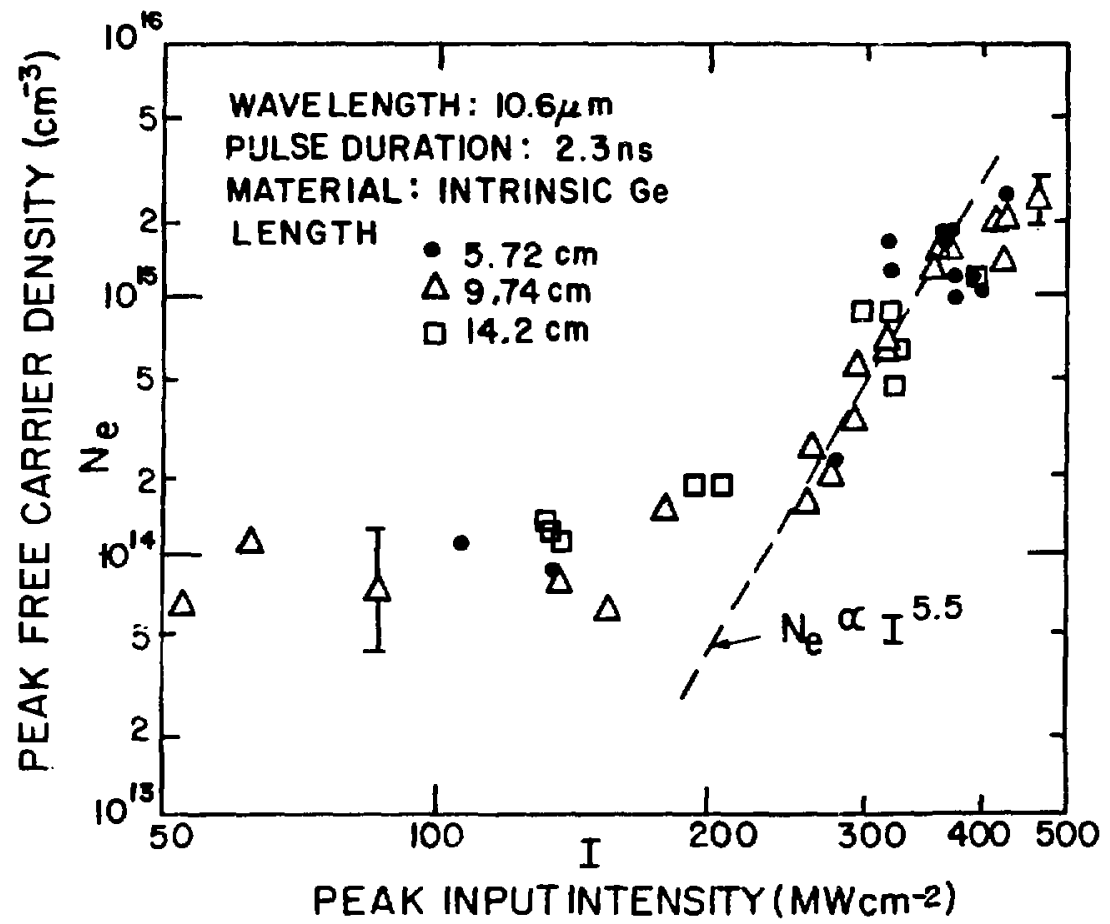

Figure VI-1. Free-carrier density as a function of $\mathrm{CO}_{2}$ 1 aser intensity in three samples of Ge (after Phipps, Ref. 8). The dashed line shows the trend of the data above the plasma-formation threshold. 
Figure 2 shows the results of DFWM experiments in two samples of Ge, each 3-mm long. One is optical grade, and the other p-type with a small-signal intensity transmission of 0.43 . This corresponds to an acceptor concentration of $6 \times 10^{15} \mathrm{~cm}^{-3}$. The plasma formation process in both these materials occurs at intensities above about $80 \mathrm{MW} / \mathrm{cm}^{2}$ in the counterpropagating-pump configuration. This reduction in the threshold for plasma formation relative to the data of $F i g .1$ is due to the enhanced intensity in the standing wave created by the counterpropagating pump beams. The peak intensity of the standing wave is $320 \mathrm{MW} / \mathrm{cm}^{2}$, well above the plasma formation threshold measured for a single beam. Above 80 $\mathrm{MW} / \mathrm{Cm}^{2}$, the reflectivity exhibits a dramatic increase. It varies approximately as the $11^{\text {th }}$ power of intensity over the range $80<I_{\text {pump }}<120 \mathrm{MW} / \mathrm{cm}^{2}$. In this regime, the presence of the free-carrier plasma could be confirmed in the optical grade sample by applying a small dc voltage across the sample and measuring the conductivity. Enhanced reflectivity is seen only in the presence of an increase in conductivity. In addition, amplification of the input sample beam by the wave-mixing process could be observed by adding a detector in this beam. I measured an increase of about $80 \%$ in this signal when the reflectivity was at its peak. Under these 


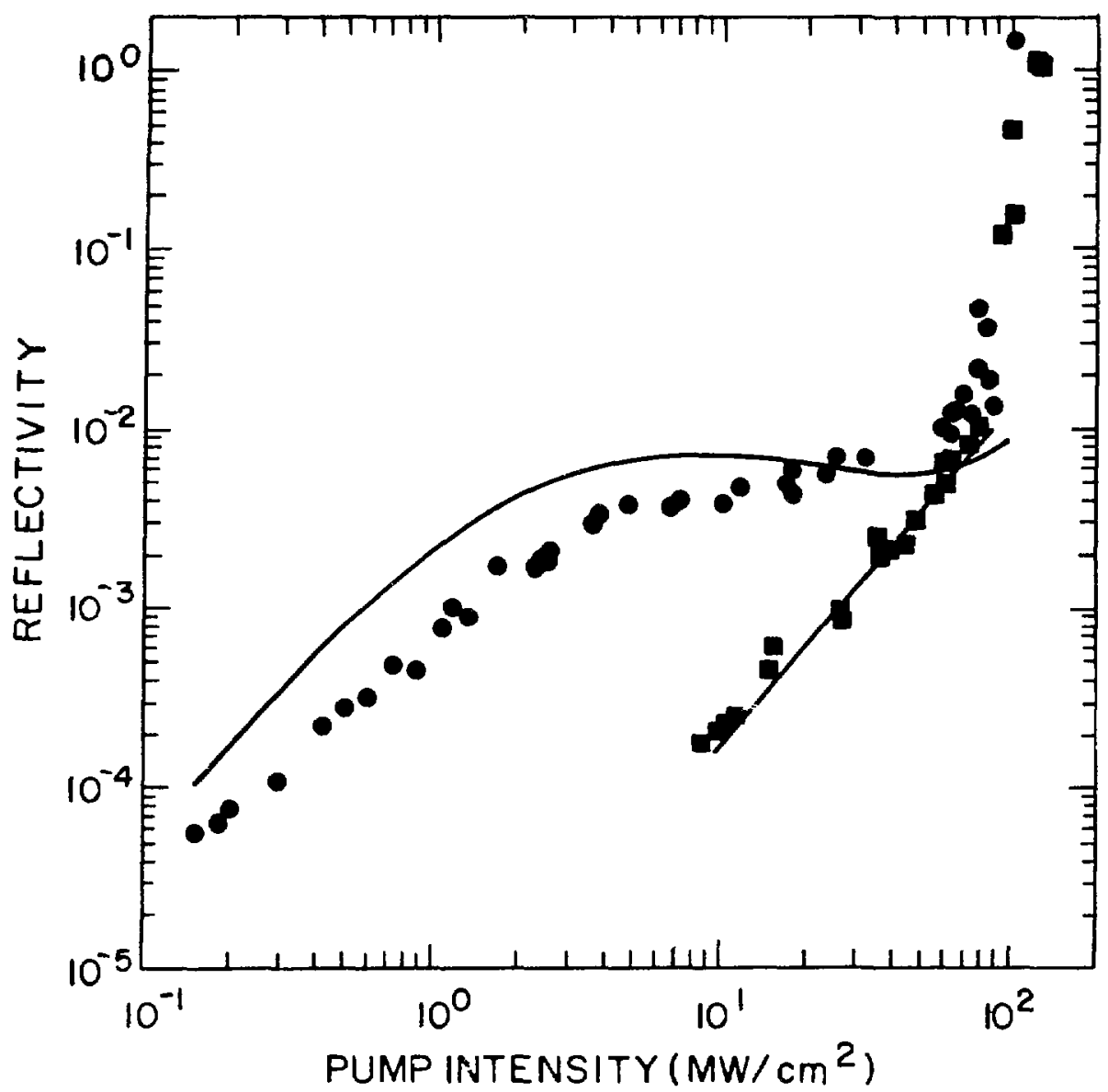

Figure VI-2. DFWM in two, 3-mm samples of Ge. The optical grade sample (squares) is modeled with Eq. 20 of Chapter III, using $x_{3}=3.2 \times 10^{-11}$ esu. The p-type sample (circles) is the same as Fig. V-5. In both samples, plasma formation occurs at about $80 \mathrm{MW} / \mathrm{cm}^{2}$. Between 80 and $120 \mathrm{MW} / \mathrm{cm}^{2}$, the reflectivity varies with the eleventh power of the pump intensity. 
conditions, a 100\% increase is expected. The difference is probably due to attenuation of the input probe beam by the medium. By using a fast detection system in the transmitted signal monitor, the onset of the plasma reflectivity could be observed. This occurs prior to the peak laser intensity and causes an abrupt increase in the transmitted signal.

The reflectivity can be modeled by calculating the change in index of refraction in the medium that is due to plasma formation, and then using the model for DFWM reflectivity first proposed by Yariv and Pepper. ${ }^{16}$

The change in index of refraction in the oresence of the free-carriers is given by the well-known formula ${ }^{7}$

$$
\Delta n=\frac{-1}{2 n_{0}} \quad \frac{4 \pi e^{2}}{m_{e} \nu^{2}}-\sum_{j} \quad N_{j} / m_{j} \text { * }
$$

where the sum extends over all carrier types, $N_{j}$ is the density of carriers of type $j$, and $m_{j}^{*}$ is the conductivity effective mass of the free carriers, normalized to the electron niass $m_{e}$. The linear index of refraction is $n_{0}$, and $v$ is the laser frequency. In the present case, there are three carrier types, with effective masses $m_{c}^{*}=0.12, m_{H}^{*}=0.31$ and $m_{L}^{*}=0.044$ for the conduction, heavy-hole and 
light-hole bands respectively. ${ }^{16}$ Using Eq. 1, I obtain $\Delta n=-\frac{2 \pi e^{2} N}{n_{0} m_{e} v^{2}}\left\{1 / m_{C} *+\left[1+\left(1+|E|^{2} / E_{S}{ }^{2}\right)^{-\frac{1}{2}}\right] / 2 m_{H} *\right.$ (4)

$$
\left.-\left[1-\left(1+|E|^{2} / E s^{2}\right)^{-\frac{1}{2}}\right] / 2 m_{L} *\right\} \text {. }
$$

Thus the change in index of refraction for say $|E|^{2} / E_{S}^{2}=$ 125 (or an intensity in the counterpropagating pumps of about $100 \mathrm{MW} / \mathrm{cm}^{2}$ ), can be found from Eq. 4 using the density from Fig. 1 of $N=2 \times 10^{15} \mathrm{~cm}^{-3}$. This gives $\Delta n=-6.4 \times 10^{-4}$ (for the purpose of comparison, the thermal index change in this case is expected to be about $\left.1 \times 10^{-5}\right)$. As noted in Chapter III, Yariv and Pepper ${ }^{18}$ obtained an expression for the reflectivity in the small-signal limit valid for equal pump beam intensities. This expression can be written $R=\tan ^{2}\left(k_{0} \Delta n L\right)$.

For our case, $k_{0}=5933 \mathrm{~cm}^{-1}$ and $L=0.3 \mathrm{~cm}$, so $R \simeq$ 5 , in reasonable agreement with the experimental value of 1. Note that this calculation ignores attenuation of the beams by the medium. Including this in the calculation has not been attempted, because it is felt that the absorption effects are too complex. For instance, the major component of the absorption saturates. As shown in the previous chapter, this saturation can lead to enhanced DFWM. However, in this case, the saturable absorption coefficient itself is modulated by the interference of the 
beams, since the generation of the carriers responsible for the saturable absorption is intensity dependent. The complexity of the nature of the absorption makes including it intractable. The absorption is not strong, less than $50 \%$, so ignoring this is probably not important. In fact, one should note that a factor-of-2 increase in the argument of the tangent would result in a prediction of infinite gain, so the fit is much more sensitive to the assumed average value of $\Delta n$ than to the attenualiun.

To demonstrate that the four-wave mixing process is dominated by the change in index of refraction in the plasma formation regime, I note that the coupling coefficient, $k$, is in general complex. The real part is proportional to the change in index of refraction, and the imaginary part is proportional to the change in the field absorption coefficient. In the simplest analysis, the reflectivity is proportional to the absolute square of k. Thus the change in index of refraction dominates the wave-mixing process if $\Delta \alpha / \Delta n k_{0} \ll 1$. For intrinsic Ge, I can estimate $\Delta \alpha \sim \alpha_{\text {tot }}$ from Eq. 2. In the present case, this gives $\Delta \alpha=0.13 \mathrm{~cm}^{-1}$ or $\Delta \alpha / \Delta i \mathrm{k}_{0}=0.034$. This results in less than a $1 \%$ change in the absolute square of $k$, so the wave mixing process is dominated by the change in index of refraction and absorption can be ignored. 
Using this simple model to predict the reflectivity over the range from $I=80 \mathrm{MW} / \mathrm{cm}^{2}$ to $120 \mathrm{MW} / \mathrm{cm}^{2}$, I find that it predicts that the reflectivity will vary with pump intensity to the lith power. This result is in excellent agreement with the experiment.

Below the plasma threshold, the reflectivities measured in these two samples are modeled with the models uiscussed earlier, for the Kerr-like effect, and/or for the saturable absorption effect, as appropriate.

The model presented above works for thin samples. As the sample thickness is increased, the additional absorption due to plasma formation results in different behavior. This can be seen in the data for intrinsic Ge presented in chapter III. As the length of the samples in Fig. III-2 (a-d), increases the enhancement of reflectivity due to plasma formation continually decreases, until for the 4-cm-long sample, the reflectivity is actually diminished by the plasma formation process. In the intrinsic samples, the maximum reflectivity observed is in the $2-c m-10 n g$ sample, where $R$ $=4$ is observed. In the p-type sample of Fig. V-4, a maximum reflectivity of 8 is observed. In this 6-mm sample, the increase in reflectivity is again modeled fairly well by the simple model presented above. 
C. The decay of a laser-induced plasma grating in Ge

The DFWM discussed in this chapter differs from the previous chapters because the gratings responsible for the scattering respond with a much slower time constant. Previous mechanisms were essentially instantaneous, responding in picoseconds to the applied fields. In the case of plasma gratings, the gratings do not disappear when the field disappears, but rather remain until eithe, the carriers diffuse into a homogeneous plasma, or they recombine. In the present case the carriers recombine at a rate that is much longer than the laser pulse duration. The effect of diffusion depends on the period of the grating, or how far the carriers must diffuse in order to obscure the grating. It is found here that the typical decay rate of a laser induced plasma grating is on the order of the laser pulse duration for the geometries I normally employ.

In this section, the measurement of the decay rate of a plasma grating is used to determine the ambipolar diffusion coefficient for the free carriers. The setup used for these measurements is diagrammed in Fig. 3 . The input laser beam is a $1.6 \mathrm{~ns}, 10.6 \mu \mathrm{m}$ pulse of about 1 J. This input beam is first split to obtain an input reference beam and a signal beam. A probe beam is also taken from the pump beam by a second splitter. The pump 
beam and the signal beam are timed to arrive simultaneously at the sample, in this case the $3-\mathrm{mm}$ piece of optical grade Ge used for Fig. 2. The calibrated attenuators are used to adjust the pump beam to about 280 $\mathrm{MW} / \mathrm{Cm}^{2}$ and the signal beam to $1 \%$ of the pump beam intensity. The interference of these two beams in the medium creates a plasma grating that is very nearly sinusoidal. This is because even though the plasma formation occurs with an $I^{5.5}$ dependence, the signal beam is relatively weak and the leading terms in this dependence will be sinusoidal in the weak interference pattern.

The probe beam that is split from the pump beam is time delayed relative to the other beams, and its polarization is rotated by $90^{\circ}$. This allows two things. First, the probe beam can be reinjected along the path of the pump beam by means of a polarization splitter, which reflects p-polarization and transmits s-polarization. Second, when the probe beam is scattered by the grating into the direction of the signal beam, the signal beam can be discriminated against by means of a polarizer, and only the scattered beam detected. The time delay of the probe beam is variable. Thus one can measure the efficiency of the grating as a function of time and deduce the decay rate of the grating. (Note that I am using a slightly 


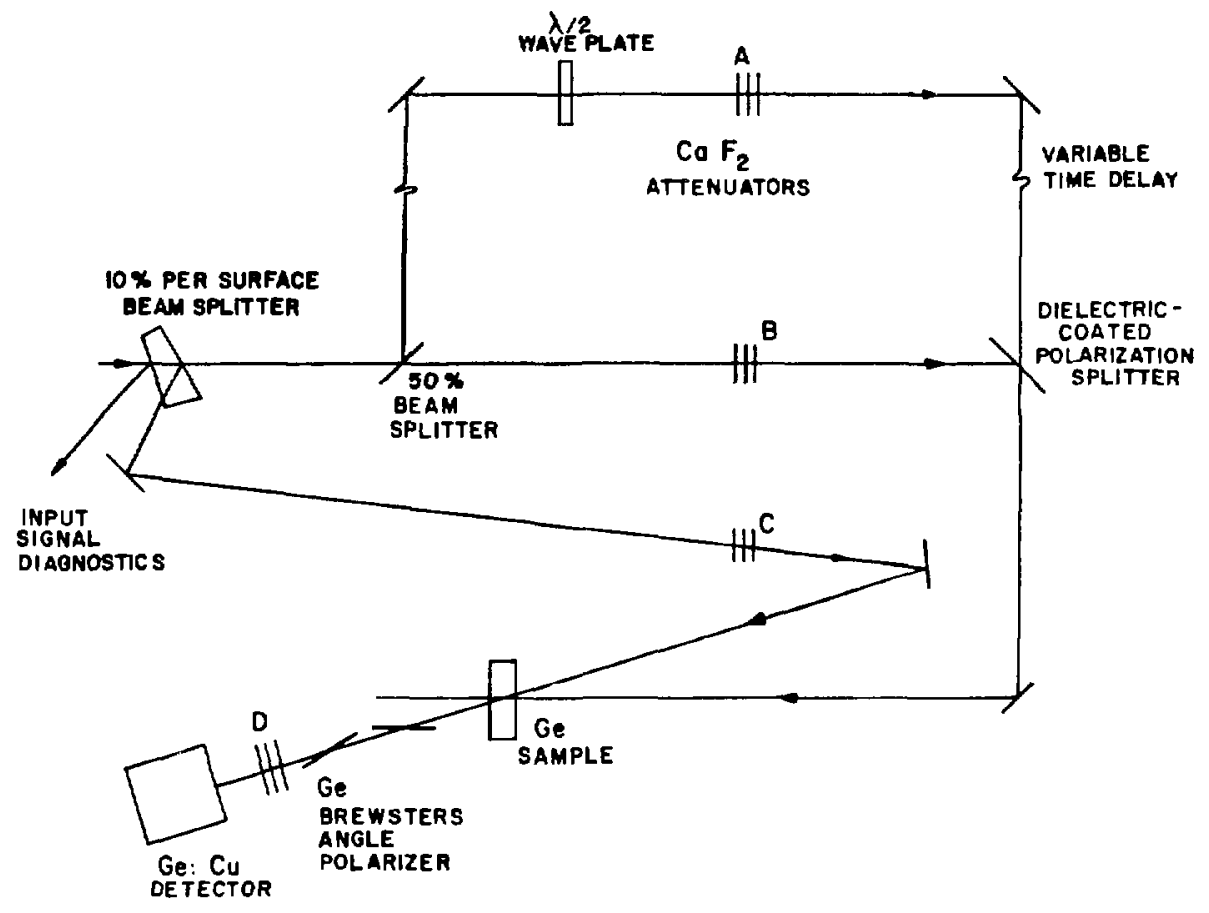

Figure VI-3. Illustration of experiment for laser-induced grating decay measurements. A free-carrier grating is induced by the interference of a signal and a pump beam in the Ge. This grating scatters a delayed probe beam into the direction of the signal. This probe is polarized perpendicular to the signal, and passes through the polarizer to the detector. By varying the time delay, the scattering efficiency of the laser induced grating as a function of time can be observed. 
different terminology here, to distinguish the signal from the delayed probe.)

A plot of the experimental scattering efficiency in arbitrary units versus the time delay is given in Fig. 4. Each point on the plot is the average of five laser shots. The error bars are two standard deviations long. The point at 20 ns is dominated by background scattering from the plasma. The amount of background scatter is determined by blocking the signal beam and measuring the scattered intensity for each time delay. From the remaining data, a least squares fit to an exponential yields a decay time for the grating of $5.4 \pm 0.9 \mathrm{~ns}$. This decay time is much shorter than the recombination time, and confirms that the dominant mechanism for decay of the grating is diffusion.

The grating period is determined by burning a piece of film at the surface of the sample. The interference pattern is then visible under a microscope, and the spatial frequency can be determined accurately. In the present case, I measured a grating period of $\Lambda=39.2 \mu \mathrm{m}$.

For a sinusoidal grating of free carriers of period $\Lambda$ and decay time $\tau$, the ambipolar diffusion coefficient is given by 18

$$
D_{a}=\frac{\Lambda^{2}}{4 \pi^{2} \tau}
$$




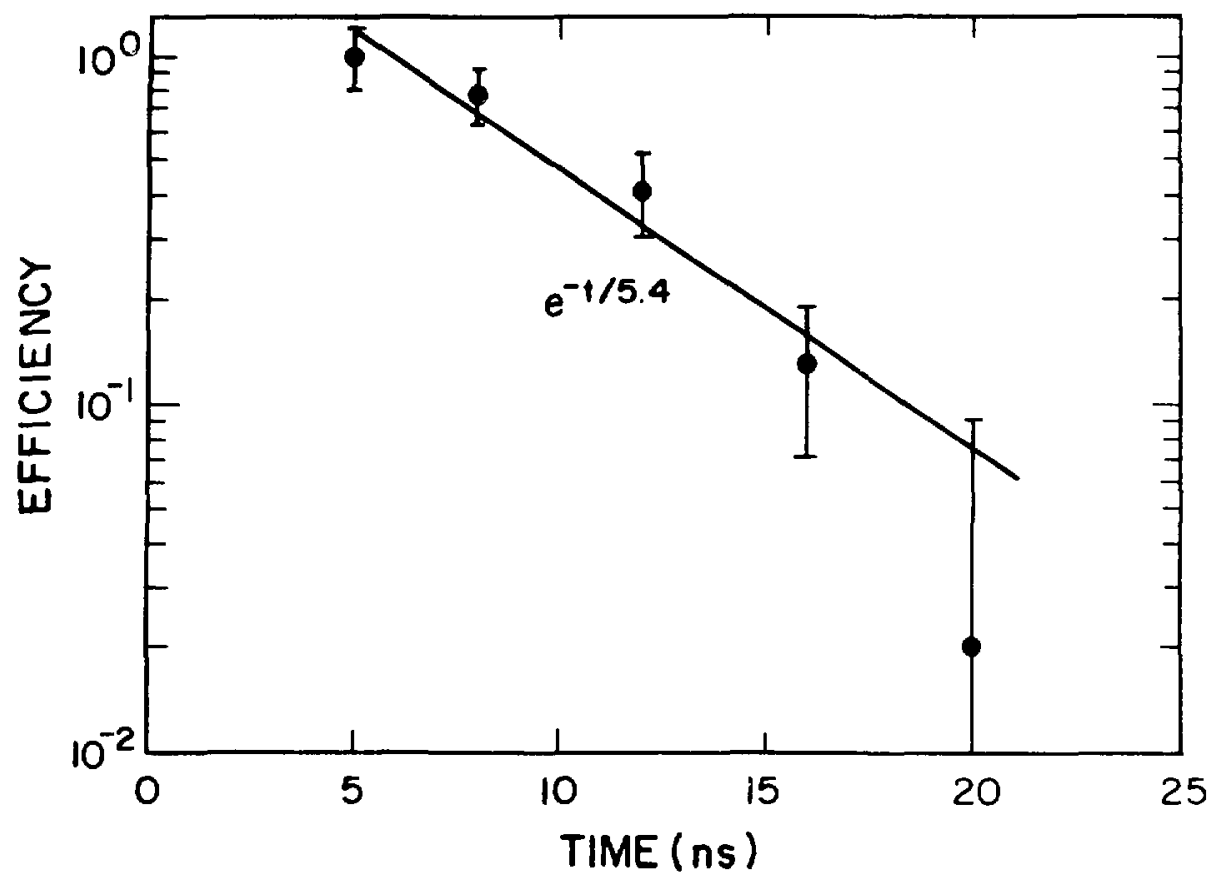

Figure VI-4. Scattering efficiency of a laser-induced grating is a $3-\mathrm{mm}$ sample of Ge as a function of time. The sample is the same as in Fig. 2. Scattering efficiency is measured in arbitrary units, and each point represents the average of five laser shots. The exponential curve is a least-squares fit to the data. 
Using this expression, I obtain from my measurements $D_{a}=72 \pm 12 \mathrm{~cm}^{2} / \mathrm{s}$. The ambipolar diffusion coefficient is given in terms of the electron and hole diffusion coefficients by

$$
D_{a}=\frac{2 D_{e} D_{h}}{D_{e}+D_{h}} .
$$

The electron and hole diffusion coefficients at room temperature in Ge are ${ }^{19} D_{e}=101 \mathrm{~cm}^{2} / \mathrm{s}$ and $D_{h}=49 \mathrm{~cm}^{2} / \mathrm{s}$, giving $D_{a}=66 \mathrm{~cm}^{2} / \mathrm{s}$. The value deduced from my measurements is in good agreement with this value.

This result confirms my expectation that the free carriers are rapidly thermalized, since the carriers diffuse with a room temperature diffusion coefficient. This is expected because the heating of the Ge by the laser pulse is known to be minimal. Even if the entire pump beam energy were to be deposited in the $3-m m-t h i c k$ sample, the temperature would increase only by $0.5^{\circ} \mathrm{K}$. The result also confirms that the diffusion is controlled by interactions of the free carriers with the lattice, and not by electron-electron or hole-hole interactions, since the carrier-lattice interactions dominate the diffusion process at the temperature and density experienced here. 
D. Conclusion

In this chapter I have presented the results of an investigation of DFWM in the plasma formation regime of Ge. This investigation resulted from the serendipitous observation of dramatically enhanced wave-mixing in an experiment on DFWM in p-type Ge. The experimental results have been satisfactorily explained by a model based on the nonlinear index of the plasma formation process. The diffusion of the carriers has been shown to obey the expected ambipolar diffusion behavior.

The principal difference between the investigation undertaken here and previous investigations is the nature of the generation of the free carriers. In all previous studies, the laser has been tuned to the tand gap of the semiconductor. In the study presented here, the laser is far from resonance with the band gap and the process for formation of the free-carrier plasma is a cascade of e lectrons. 
Footnotes

1. R. K. Jain, M. B. Klein and R. C. Lind,

"High-efficiency degenerate four-wave mixing of 1.06 um radiation in silicon," Optics Letters $\underline{4}, 328$ $(1979)$.

2. J. P. Woerdman, "Formation of a transient free-carrier hologram in Si," Optics Communications 2,212 (1971).

3. M. A. Khan, P. W. Kruse, and J. F. Ready, "Optical phase conjugation in $\mathrm{Hg}_{1-x_{x}} \mathrm{Cd}_{x} \mathrm{Te}$, "Optics Letters $\underline{5}, 261(1980)$.

4. R. K. Jain and D. G. Stee 1, "Degenerate four-wave mixing of 10.6 um radiation in $\mathrm{Hg}_{1-x} \mathrm{Cd}_{\mathrm{x}} \mathrm{Te}$," Applied Physics Letters 37, 1 (1980).

5. D. A. B. Miller, R. G. Harrison, A. M. Johnston, C. T. Seaton, and S. D. Smith, "Degenerate four-wave mixing in InSb at $5^{\circ} \mathrm{K}, "$ optics Communications 32,478 (1980).

6. D. C. Haueisen and D. Depatia, private communication.

7. R. K. Jain and M. B. Klein, "Degenerate four-wave mixing near the band gap of semiconductors, "Applied Physics Letters 35 , 454 (1979).

8. C. R. Phipps, Jr. and S. J. Thomas, unpublished.

9. S. Y. Yuen, R. L. Aggarwal, N. Lee, and B. Lax, "Nonlinear absorption of $\mathrm{CO}_{2}$ laser radiation by nonequilibrium carriers in germanium," Optics Communications 28, 237 (1979), and S. Y. Yuen, R. L. Aggarwa 1, and B. Lax, "Saturation of transmitted intensity of $\mathrm{CO}_{2}$ laser pulses in Ge," Journal of Applied Physics $\underline{5} 1,1146$ (1980).

10. Y. Danileiko, A. Epifanov, T. Lebedeva, A. Manenkov, V. Milyaev, and S. Sidorin, Reprint No. 10,

P. N. Lebedev, Physical Institute, Moscow (1977).

11. R. B. James, private communication.

12. A detailed description of Phipp's work has not been published. The discussion presented here also appears in D. E. Watkins, C. R. Phipps, Jr. and S. J. Thomas, "Observation of amplified reflection through degenerate four-wave mixing at $\mathrm{CO}_{2}$ laser wavelengths in germanium," Optics Letters 6, 76 (1981). 
13. R. A. Smith, Semiconductors (Cambridge University Press, Cambridge, 1968) p. 350.

14. F. Keilmann, "Infrared saturation spectroscopy in p-type germanium," IEEE Journal of Quantum Electronics $\underline{Q E-12}, 592(1976)$.

15. H. B. Briggs and R. C. Fletcher, "Absorption of Infrared light by free carriers in germanium," Physical Review 9l, 1342 (1953).

16. B. Lax and J. G. Mavroides, "Statistics and galvano-magnetic effects in germanium and silicon with warped energy surfaces," Physical Review 100, 1650 $(1955)$.

17. A. Yariv and D. M. Pepper, "Amplified reflection, phase conjugation, and oscillation in degenerate four-wave mixing," Optics Letters 1, 16 (1977).

18. J. P. Woerdman, "Some optical and electrical properties of a laser-generated free-carrier plasma in silicon," Philips Research Reports Supplements 7, 1 $(1971)$.

19. See Ref. 12 and E. Burstein, et a 1., "Optical and Photoconductive properties of silicon and germanium," in Photoconductivity Conference 1954 (Wiley, New York, $195 \overline{4)}$. 


\section{CHAPTER VII}

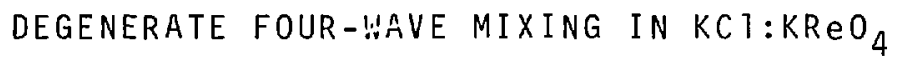

\section{Introduction}

In this chapter, both theoretical and experimental investigations of degenerate four-wave mixing in $\mathrm{KCl}: \mathrm{KReO}_{4}$ are discussed. The study of DFWM in this medium was prompted by the observation that it behaved as an ideal two-level saturable absorbar, ${ }^{1}$ and the publication of a simple theory by Abrams and Lind for DFWM in two-level systems. ${ }^{2}$ In my investigations of this material, I have found that both of these ideas have to be modified. The simple theory of DFWM in a two-level system is inadequate because it ignores absorption of the pump beams by the medium. In the specific case of DFWM in KCl:KReO4, the two-level model is replaced by a three-level model to account for discrepancies between theory and experiment at high intensities.

This chapter is divided into five sections as follows: The first section describes the ReO- ion and discusses the spectroscopy of this ion when it is substitutionally doped into KCl. In $\operatorname{section} B, a$ three-level model for the polarizability of KCT:KReO 4 is derived. This model is treated in the fashion of chapter IV to obtain a model for DFWM in section $C$. Section $D$ 
compares the model to experimental results, and section $E$ gives a discussion and conclusion.

\section{A. A description of $\mathrm{KCl}: \mathrm{KReO}_{4}$}

The $\mathrm{ReO}_{4}^{-}$ion is a tetrahedral, monovalent, spherical-top molecule. As such, it has two normal modes of vibration which are infrared active ${ }^{3}$ These are designated $v_{3}$ and $v_{4}$. The frequency of the $v_{3}$ mode is near the $10-\mu m$ band of the $\mathrm{CO}_{2}$ laser, and is the mode of interest here. The molecule is diagrammed in Fig. 1. Cartesian coordinates may be formed by connecting opposite midpoints of the edges of the tetrahedron so that the Re is at the origin. In this coordinate system, the $v_{3}$ vibrational mode is one where the Re oscillates oppositely to all four oxygen atoms along any one of the three cartesian axes. The three-fold degeneracy of this mode of oscillation is readily apparent. The oscillations of the free radical, $\operatorname{Re}_{4}^{-}$, are essentially harmonic in nature.

When the $\mathrm{ReO}_{4}^{-}$ion is doped into $\mathrm{KCl}$, the molecule replaces a $\mathrm{Cl}^{-}$ion in the crystal. The site symmetry in the crystal remains cubic, but because the $\mathrm{ReO}_{4}^{-}$ion is larger than the $\mathrm{Cl}^{-}$ion, the local lattice undergoes compression. The result of this compression is a shift of the room-temperature resonant frequency of the $\operatorname{ReO}_{4}^{-} v_{3}$ 


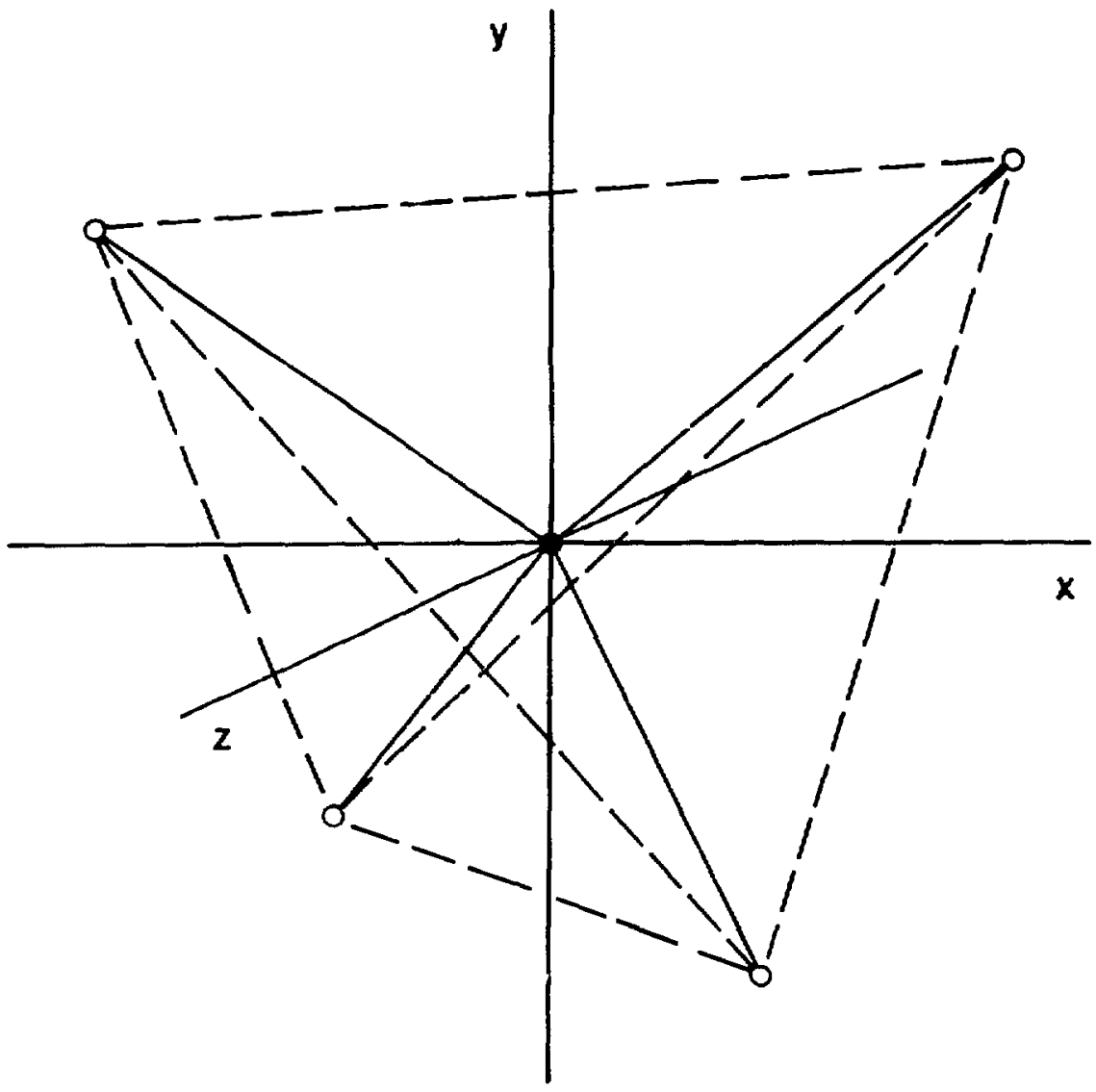

Figure VII-1. Diagram of the Re04 molecule (after Ref. 3). The Re is at the center and the four oxygen atoms are at the corners of a tetrahedron, with radius $3.09 \AA$. The $v_{3}$ vibrational mode is one where the Re oscillates opposite to all four oxygens along any one of the three cartesian coordinates. 
vibrational mode into coincidence with the $P-28$ line of the $10-\mu \mathrm{m}$ band of the $\mathrm{CO}_{2}$ laser, and the introduction of a large anharmonicity into the vibrational mode.

Another major effect on the vibrational mode of the ion in the $K C l$ lattice is a large increase in both the dephasing rate and the energy relaxation rate of the excited vibrational states. This is due to interactions between the vibrational modes of $\mathrm{KCl}: \mathrm{KReO}_{4}$ and the phonons of the KCl lattice.

Both the ground state spectrum and the excited state spectrum have been measured by Ahrenkiel et al ${ }^{1}$ at LoS Alamos National Laboratory. The ground state absorption spectrum is shown in Fig. 2. Note that the transition is essentially coincident with $\mathrm{P}-28$ and that the full width at half maximum of the absorption is about $1 \mathrm{~cm}^{-1}$. Ahrenkiel reports a value of $.98 \mathrm{~cm}^{-1}$, corresponding to a dephasing time of $\mathrm{T}_{2}=10.8 \mathrm{ps}$. He also reports an energy relaxation time of $T_{1}=122 \mathrm{ps}$.

Saturation of the ground state transition by intense laser radiation has also been observed. This is shown in Fig. 3. The curve is a fit to the data by Phipps of a two-level homogeneously broadened saturable absorption model ${ }^{4}$ which includes a small nonsaturable component. From such fits, a value of $I_{s}=1.45 \mathrm{MW} / \mathrm{cm}^{2}$ has been obtained for the room-temperature saturation intensity. 


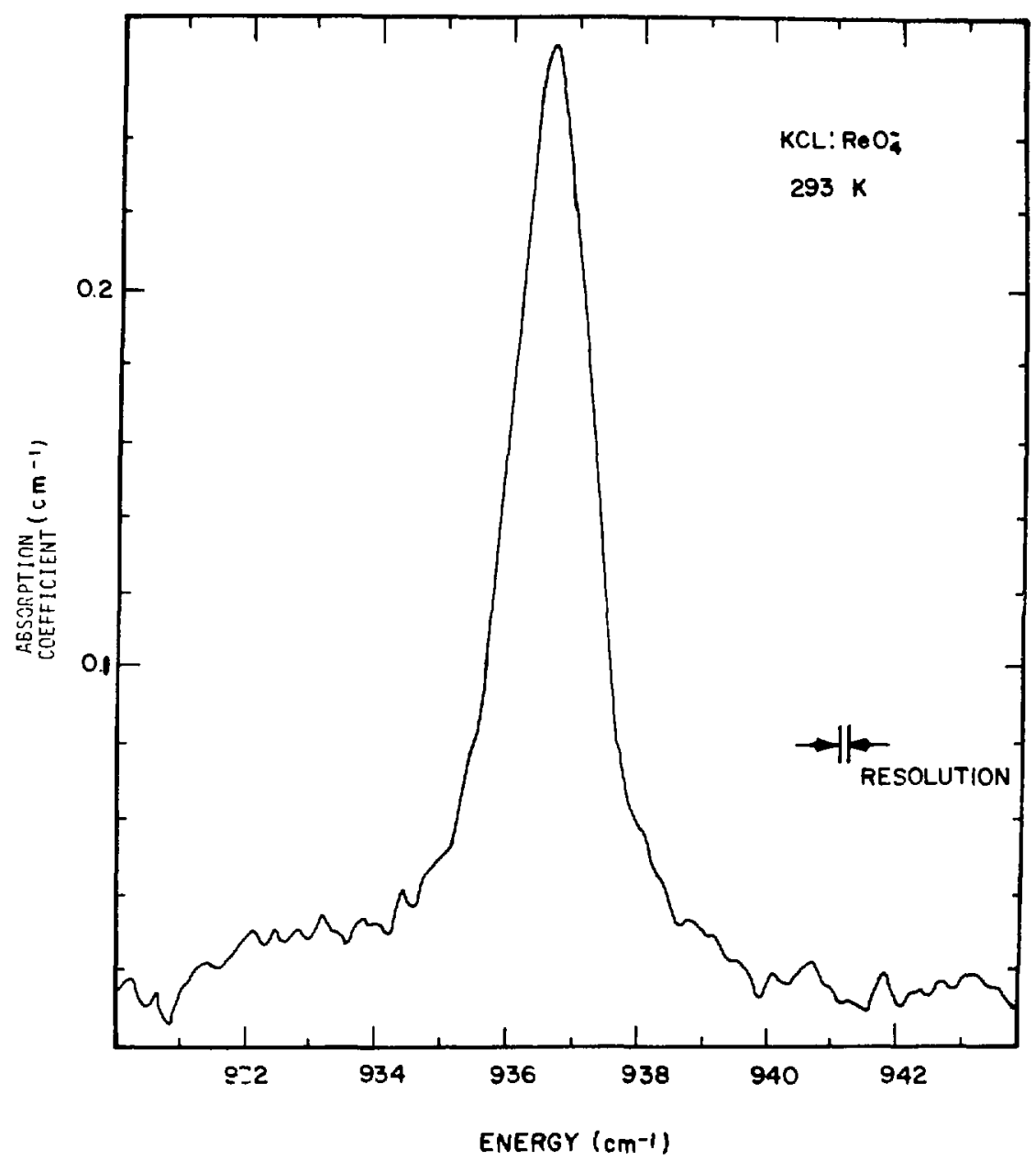

Figure VII-2. Absorption coefficient as a function of photon energy in KCl: $\mathrm{KReO}_{4}$ (after Ref. 1). The peak is coincident with $\mathrm{P}-28$ of the $10 \mu \mathrm{m}$ band of the $\mathrm{CO}_{2}$ laser. The full width at half maximum is $0.8 \mathrm{~cm}^{-1}$. 


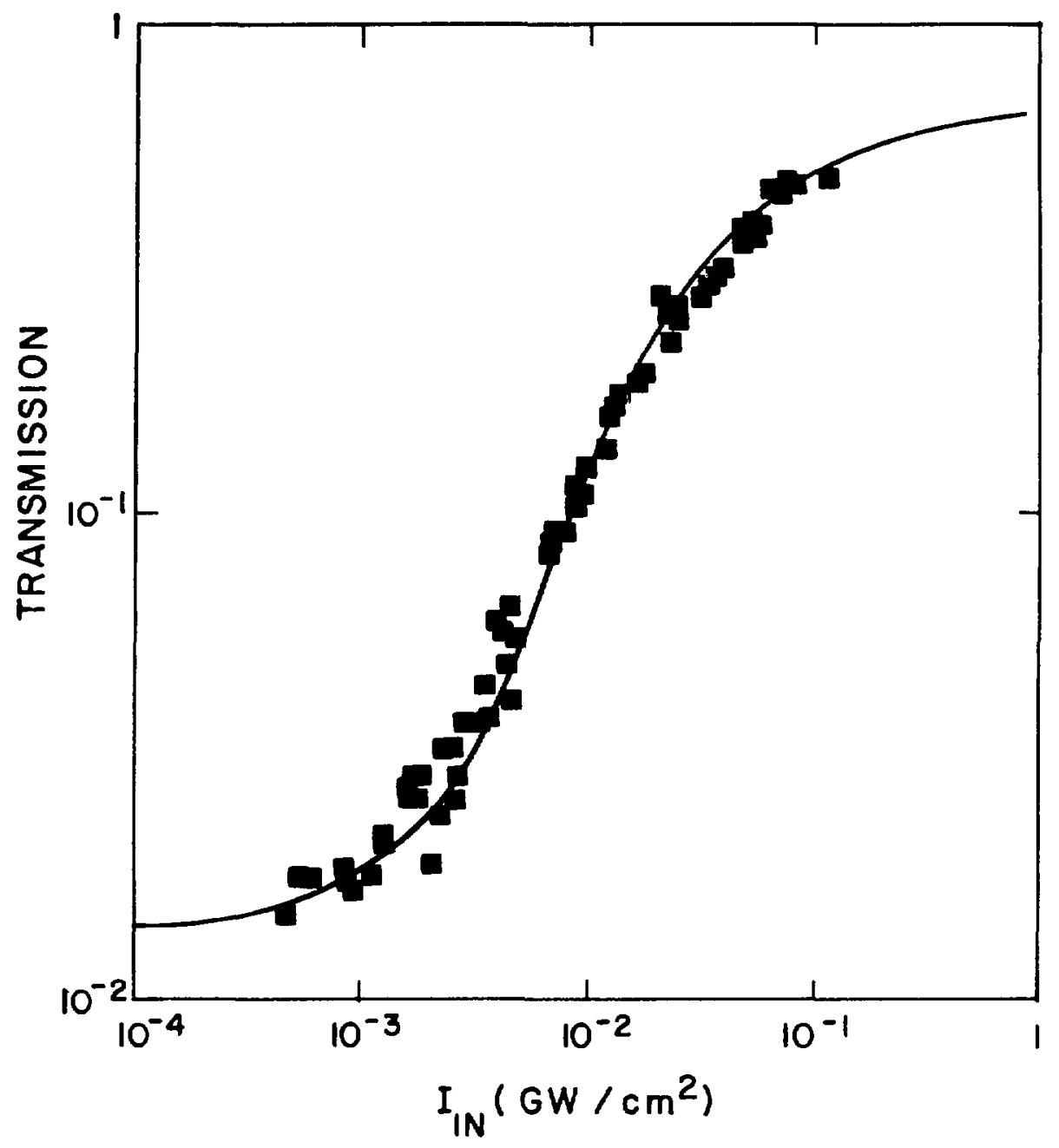

Figure VII-3. Saturation of the optical transmission of $\mathrm{KCl}: \mathrm{KReO}_{4}$ (after Ref. 4). The curve is a fit of a two-level saturable absorption model using a saturation intensity of $\mathrm{I}_{\mathrm{S}}=1.45 \mathrm{MW} / \mathrm{cm}^{2}$. 
The observation that this medium saturates like a two-level system is explained by the anharmonicity of the $v_{3}$ vibrational mode. The anharmonicity produces a red shift in the spectrum of the higher vibrational states. Thus, although the transition from the ground state to the first excited state is resonant with $P-28$, the transition from the first excited state to the second is shifted off resonance from $\mathrm{P}-28$. This has been confirmed by excited state absorption experiments performed by Ahrenkiel, who measured the induced absorption at frequencies to the red of P-26 in the presence of intense saturating $P-26$

radiation in a cooled sample of $\mathrm{KCl}_{\mathrm{KReO}} .^{5}$ The energy diagram of Fig. 4 deduced from this data. The transition from the first to the second excited state is red shifted by $3.8 \mathrm{~cm}^{-1}$. It is assumed that the change in resonant frequency with temperature for both $0-1$ and the 1-2 transitions are the same, so that the same red shift of $3.8 \mathrm{~cm}^{-1}$ applies at room temperature where I work. At room temperature, I take $v_{3}(0,1)=936.7$ $\mathrm{cm}^{-1}$ and $v_{3}(1,2)=932.9 \mathrm{~cm}^{-1}$. Figure 4 forms the basis of the three-level model presented in the next section.

B. Polarizability of a three-level system For a two-level system, it is possible to solve the Schroedinger equation and determine the polarizability 
$\mathrm{ReO}_{4}^{-} \quad \nu_{3}$ TRANSITION

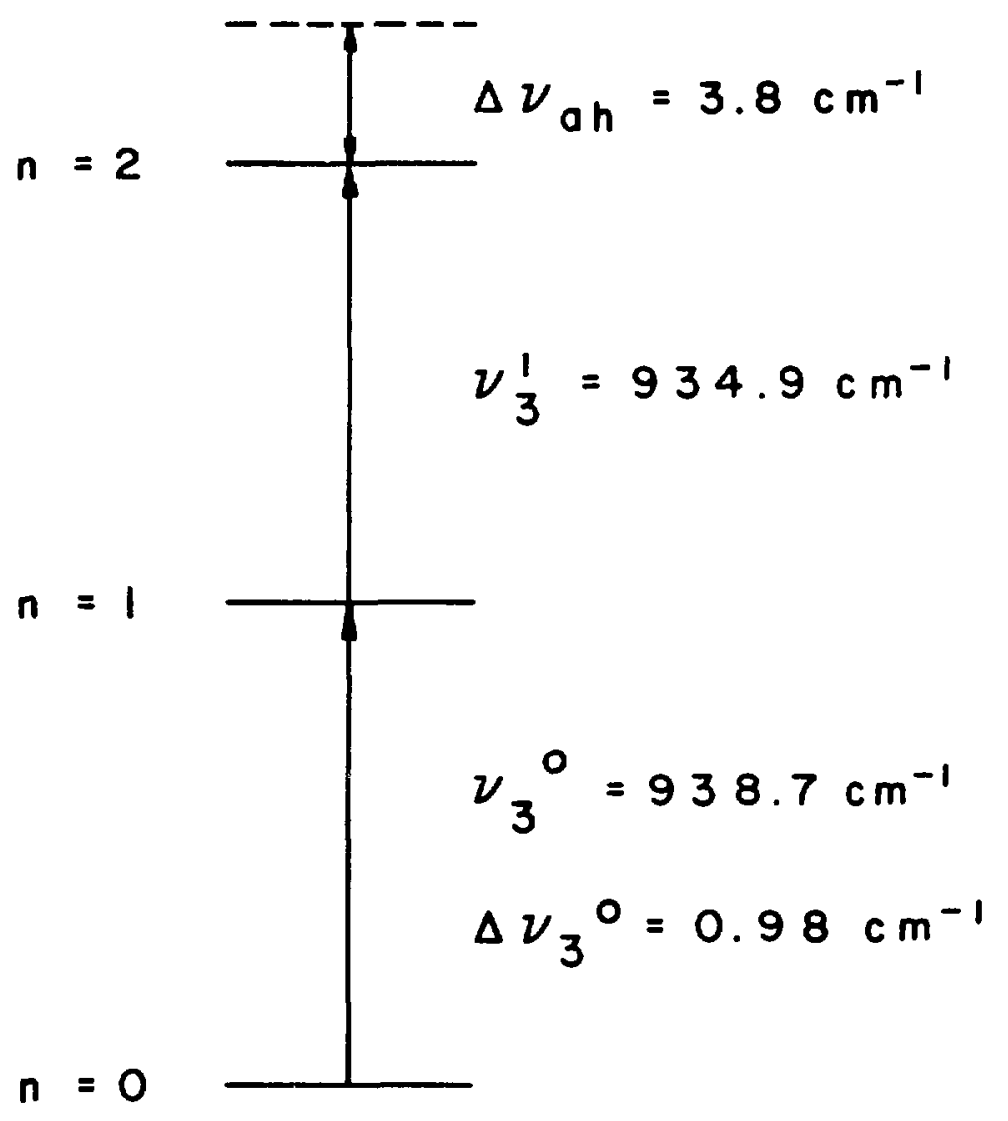

Figure VII-4. Energy diagram of the $v_{3}$ vibrational spectrum of $\mathrm{KCl}: \mathrm{KReO}_{4}$ at $105^{\circ} \mathrm{K}$ (see Ref. 5). The anharmonic shift introduced by interaction with the KCl 1 attice is $3.8 \mathrm{~cm}^{-1}$. This figure forms the basis of a three-level model developed in this chapter. 
using only the rotating wave approximation. ${ }^{6}$ Although in principle it is possible to extend this solution to 3 or $n$ levels, in practice this is not possible. The reason is that to solve an $n-l e v e l$ system in this approximation, one must essentially obtain the determinant of an $\left(n^{2}-1\right) \times$ $\left(n^{2}-1\right)$ matrix. Such a determinant has $\left(n^{2}-1\right)$ : terms. For a two-level system, six terms are involved, but for a three-level system, there are 40,320 terms. Thus, the direct approach to the problem of obtaining the polarizability is analytically intractable. In this section, a perturbation analysis is used to obtain the polarizability of a three-level system such as is shown in Fig. 4 .

The perturbation analysis used here treats the third level as a perturbation on a two-level system. The third level is assumed to be well removed from resonance, so that the population of ions in this state is negligible. I follow the procedure of Narducci et al, ${ }^{7}$ who treated a system near a two-photon resonance with the intermediate states well removed from resonance.

The Hamiltonian of the three-level system is taken as

$$
H=E_{0} \mid 0>\left\langle 0\left|+E_{1}\right| 1>\left\langle 1\left|+E_{2}\right| 2><2\right|-\vec{p} \cdot \vec{\varepsilon}\right.
$$

where $E_{j}$ is the energy of the $j$ th state. For a linearly 
polarized electric field, $\vec{p} \cdot \vec{\varepsilon}=p \in$ where the dipole moment operator is

(2) $\quad p=|0><1| \mu_{01}+|1><2| \mu_{12}+|1><0| \mu_{10}+|2><1| \mu_{21}$.

The state of the three-level system in the presence of the exciting field can be expanded in terms of the eigenstates of the unperturbed Hamiltonian;

$$
|\psi\rangle=c_{0} e^{-i \omega_{0} t}|0\rangle+c_{1} e^{-i \omega_{1} t}|1\rangle+c_{2} e^{-i \omega_{2} t}|2\rangle,
$$

where $\omega_{j}=E_{j} / \hbar$. Substituting this into the Schroedinger equation,

$$
\text { in } \frac{\partial}{\partial t}|\psi>=H| \psi>\text {, }
$$

and employing the orthogonality of the unperturbed eigenstates, equations of motion for the amplitudes are obtained:

$$
\dot{C}_{0}=\frac{i}{\hbar} \mu_{01} C_{1} \in e^{i \omega_{0} t},
$$

$$
c_{1}=\frac{i}{\hbar} \mu_{10} C_{0} \varepsilon \mathrm{e}^{i \omega} 10 t+\frac{i}{\hbar} \mu_{12} C_{2} \varepsilon \mathrm{e}^{i \omega} 12 t
$$

and

$$
\dot{C}_{2}=\frac{i}{\hbar} \mu_{21} C_{1} \varepsilon \mathrm{e}^{\left.i \omega_{2}\right\} t},
$$

where $\omega_{i j}=\omega_{i}-w_{j}$. In the perturbation treatment, the last of these equations may be integrated 
directly to obtain the amplitude $C_{2}$. To do this, I substitute for the field

$$
\varepsilon=\frac{1}{2}\left(E(r, t) e^{-i \nu t+E *(r, t)} e^{i v t),}\right.
$$

and assume the slowly varying envelope approximation. In this approximation, I replace $C_{1}$ and $E$ with their values at the upper limit integration, $t$, and remove them from the integral. Then

$$
c_{2}=\frac{1}{2 h} \mu_{21} c_{1}\left\{\frac{E^{*}\left(e^{i\left(\omega_{2}+v\right) t}-1\right)}{\omega_{21}+v}+\frac{E\left(e^{i\left(\omega_{2} 1-v\right) t}-1\right)}{\omega_{21}-v}\right\}
$$

This value for $C_{2}$ is substituted into Ens. 4 and I transform to the interaction picture via $c_{j}=c_{j} \exp \left(i \omega_{j} t\right)$. This gives

$$
c_{0}=-i \omega_{0} c_{0}+\frac{i}{2 n}-\mu_{0} c_{c}\left(E e^{-i \nu t}+E * e^{i \nu t}\right)
$$

(7b)

$$
\begin{aligned}
& c_{1}=-i \omega_{1} c_{1}+\frac{i}{2 \hbar} \mu_{10} c_{0}\left(E e^{-i v t}+E^{*} e^{i v t}\right) \\
& +\frac{i}{4 \pi 2}\left|\mu_{12}\right|^{2} c_{1}\left\{\frac{E \star^{2}\left(e^{2 i \nu t_{-}}-i\left(\omega_{2} 1-v\right) t\right.}{\omega_{21}+v}\right) \\
& +\frac{E^{2}\left(e^{-2 i v t}-e^{-i\left(\omega_{2} 1+v\right) t}\right)}{\omega_{2 l}-v} \\
& +|E|^{2}\left[\frac{1-e^{-i\left(\omega_{2} 1^{+v}\right) t}}{\omega_{21}+v}\right.
\end{aligned}
$$

152 


$$
\left.\left.+\frac{1-e^{-i\left(\omega_{2} 1-v\right) t}}{\omega_{21}-v}\right]\right\}
$$

I form the density matrix by taking the products $\rho_{i j}=c_{i} c_{j}{ }^{*}$. Then, assuming the steady state, $\rho_{i j}=0$, and negligible population in the third level, $\rho_{00}+\rho_{11}=1$, and introducing the dipole dephasing time $\mathrm{T}_{2}$ phenomenologically, I obtain the following equation of motion for the off diagonal matrix element:

$$
\begin{aligned}
\dot{\rho}_{01}=-\rho_{01} / T_{2}-i \omega_{10} \rho_{01} & \\
& +\frac{i}{2 i}-\mu_{01}\left(E e^{\left.-i \nu t+E e^{i \nu t}\right)\left(\rho_{1} 1-\rho_{00}\right)}\right. \\
& -\frac{i}{4 \pi^{2}}\left|\mu_{12}\right|^{2} \rho_{01}\left\{\begin{array}{l}
\text { conjugate of } \\
\text { bracket contents } \\
i n \text { Eq. 7b }
\end{array}\right\} .
\end{aligned}
$$

This can be solved in the rotating wave approximation.

I put

$$
E_{s}^{2}=\frac{\hbar^{2}}{T_{1} T_{2}\left|\mu_{01}\right|^{2}}
$$

$$
\left.\delta=(\nu-\omega)+\gamma|E|^{2} / 2 \mathrm{~T}_{2} \mathrm{E}_{\mathrm{s}}{ }^{2}\right) \mathrm{T}_{2} \text { (normalized detuning), }
$$

and

$$
Y=\frac{\left|\mu_{2}\right|^{2}}{\left|\mu_{0}\right|^{2} \mid} \frac{\omega_{2} \mid}{T_{1}\left(\left.\omega_{2}\right|^{2}-v^{2}\right)} \quad \text { (a unitless parameter). }
$$


Then by substituting $\rho_{01}=\rho \exp (i v t)$ and equating coefficients of the exponential, I find

$$
\rho_{01}=\frac{\left(i T_{2} / 2 \hbar\right) \mu_{01}\left(2 \rho_{11}-1\right) E * e^{i v t}}{(1+i \delta)}
$$

Because of my assumptions, the only remaining element of the density matrix that I need to determine is $\rho_{11}$. Its equation of motion is

$$
\rho_{11}=-\rho_{11} / T_{1}+\frac{i}{2 \hbar} \mu_{10}\left(E e^{-i \nu t}+E^{*} e^{i \nu t}\right) \rho_{01}
$$

$$
\begin{aligned}
& -\frac{i}{2 \hbar} \mu_{01}\left(E e^{-i v t}+E^{\star} e^{i \nu t}\right) \rho 10 \\
& +\frac{i}{2 \hbar 2}\left|\mu_{12}\right|^{2} \rho 11 \text { Im }\{\text { same as Eq. } 7 b\}
\end{aligned}
$$

where the energy decay time $T_{1}$ has now also been introduced phenomenologically. Using the steady state assumption and the solution for $\rho_{01}$, this yields

$$
\rho_{11}=\frac{|E|^{2} / 2 E s^{2}}{1+\delta^{2}+|E|^{2} / E s^{2}}
$$

Finally, the polarization is given in the interaction picture by

$$
\begin{aligned}
P & =N\langle\psi|p| \psi\rangle \\
& =N\left[\mu_{01} \rho_{10}+\mu_{10} \rho_{01}\right]
\end{aligned}
$$




$$
\begin{aligned}
+\frac{N|\mu 12|^{2}}{2} \rho_{11\{} & {\left[\frac{E^{*}\left(e^{i v t}-e^{-i \omega_{2} 1 t}\right)}{\omega_{21} 1^{+v}}\right.} \\
& \left.\left.+\frac{E\left(e^{-i v t}-e^{-i \omega_{2} t}\right)}{\omega_{21}-v}\right]+c . c \cdot\right\}
\end{aligned}
$$

where $N$ is the number density of three-level systems. Taking the Fourier component of $P$ at the frequency of the driving field gives

$$
\begin{aligned}
& P(\nu)=\frac{n^{2} \alpha_{0}}{2 \pi k}\left\{-\frac{(i+\delta) E}{\left(1+\delta^{2}+|E|^{2} / E_{s}\right)}\right. \\
& \left.+2 \gamma \frac{T_{1}}{T_{2}} \frac{\left(|E|^{2} / E_{s}^{2}\right) E}{\left(1+\delta^{2}+|E|^{2} / E_{s}^{2}\right)}\right\}
\end{aligned}
$$

where

$$
\text { (17) } \quad \alpha_{0}=N \pi k T_{2}\left|\mu_{01}\right|^{2} / n n^{2}
$$

and $n$ is the index of refraction of the host material, in this case the $K C l$.

Some comments should be made regarding this polarizability. Note that the first term in this polarizability is identical in form to that for a two-level system as given in Chapter IV Eq. 1. There is, however, a major difference in the definition of $\delta$, which contains an additional term for the three-level system. This term describes the ac Stark effect, and introduces an intensity-dependent red shift for the first 
excited state. The second term has no analog in the two-level system and is in phase with the electrical field. Thus it affects only the phase of the propagating electromagnetic wave, and not the attenuation of the wave by the medium. This is reasonable since the underlying assumption is that there is negligible population in the third level, and hence it is not responsible for absorption of energy from the wave. Except for the minor ac Stark effect, this model describes the saturable absorption in the same way as the two-level model. of the parameters used in the derivation of the polarizability, only two are not known a priori. These are the small signal absorption coefficient $\alpha_{0}$, and the dimensionless parameter $\gamma$. The parameter $\alpha_{0}$ is not fundamental in the sense that it varies from crystal to crystal with the doping density and thus must be measured for each crystal. The parameter $\gamma$ measures the relative strength of the dipole moments $\mu_{01}$ and $\mu_{12}$, and the detuning of the driving field from resonance with the transition from the first to the second excited state. The value of the detuning can be obtained from Ahrenkiel's work, 1 or the discussion of Fig. 4 in Section $A$. The relative strength of the dipole moments can be approximated by assuming chat the anharmonic nature of the oscillator can be ignored to first order. Then the 
wavefunctions for a three-dimensional harmonic oscillator can be used to find the ratio of the dipole moments. This gives $\left|\mu_{01}\right|^{2} /\left|\mu_{12}\right|^{2}=1 / 4$, so that $\gamma=-.023$ for $P 28$ of the $10 \mu \mathrm{m}$ band of the $\mathrm{CO}_{2}$ laser.

\section{Theory of DFWM in KCl:KRe0 4}

In this section the polarizability derived in the previous section is used to model DFWM in $\mathrm{KCl}: \mathrm{KReO}_{4}$. The analysis performed here is based on the general technique given in Chapter IV. The model obtained is similar to the original theory given by Abrams and Lind, ${ }^{2}$ except that I use a more sophisticated form for the polarizability, and include the effect of pump attenuation by the medium.

DFWM in multilevel systems has been investigated by other people. Haueisen ${ }^{8}$ and Fu and Sargent ${ }^{9}$ have considered the problem of DFWM near a two-photon resonance with the intermediate state well removed from resonance. Steel and $L^{10}{ }^{10}$ have discussed DFWM experimental results in $\mathrm{SF}_{6}$ in terims of a three-level polarizability, without attempting to solve for the reflectivity. Steel, Lind, and $L_{a m}^{11}$ have treated $S F_{6}$ as a four-level system, or more accurately, as a pair of two-level systems. None of these models considers the effect of pump attenuation by the medium. They also are not applicable to the type of 
three-level system I consider where the optical frequency is near resonance with a single-photon transition.

The procedure I use is as follows: First the polarizability is expanded in the perturbation series to first order in the weak probe fields, in direct analogy to Eq. IV-2 for a two-level system. This polarizability is then spatially averaged, to obtain a form consistent with the slowly varying envelope approximation. Substituting the resulting polarizability into the corresponding zeroand first-order wave equations, I obtain two coupled equations for the pump beam amplitudes, an equation for the sum of the pump beam phases, and two coupled equations of standard form for the probe waves. The numerical technique of Chapter IV is then applied to calculate the reflectivity as a function of pump intenjity.

The first step in the procedure outlined above is to expand the polarizability in a perturbation series in $\Delta E$ and $\Delta E^{\star}$. This is done by expressing the total field as the sum of two parts, $E / E_{s}=E_{0}+\Delta E$, where $E_{0}$ and $\Delta E$ are the sums of the pump fields and the probe fields respectively. I define the detuning from the 0 to 1 transition as

$$
\delta^{\prime}=\left(\nu-\omega_{10}\right) T_{2}
$$


and the quantities $\Gamma$ and $G$ by

(19) $\Gamma=1+\delta ' \gamma$ and $G=\gamma\left(2 T_{1} / T_{2}-1 / 2\right)$

Then the zero-order term in the perturbation expansion of $P$ is

$$
P_{0}=\frac{n^{2} \alpha_{0} E_{s}}{2 \pi k} \quad \frac{\left(i-\delta^{\prime}+G\left|E_{0}\right|^{2}\right) E_{0}}{D}
$$

and the first-order term is

$$
\begin{aligned}
& \Delta P=\frac{i n^{2} \alpha_{0} E_{S}}{2 \pi k D^{2}}\left(1+\delta r^{2}-\gamma^{2}\left|E_{0}\right|^{4} / 4\right) \Delta E \\
& +\frac{n^{2} \alpha_{0} E_{S}}{2 \pi k D^{2}}\left\{-\hat{o}^{\prime}\left(1+\delta^{\prime 2}\right)+2 G\left(1+\delta^{\prime 2}\right)\left|E_{0}\right|^{2}\right. \\
& \left.+\left(\Gamma G+\delta^{\prime} \gamma^{2} / 4\right)\left|E_{0}\right|^{4}\right\} \Delta E \\
& -\frac{i n^{2} \alpha_{0} E_{S}}{2 \pi k D^{2}}\left(\Gamma+\gamma^{2}\left|E_{0}\right|^{2 / 2}\right) E_{0}{ }^{2} \Delta E * \\
& +\frac{n^{2} \alpha_{0} E_{S}}{2 \pi k D^{2}}\left\{G(1+\delta \cdot 2)+\delta \cdot \Gamma+\gamma^{2} \delta \cdot\left|E_{0}\right|^{2} / 2\right. \\
& \left.-\gamma^{2} G\left|E_{0}\right|^{4} / 4\right\} E_{0}^{2} \Delta E *
\end{aligned}
$$

where the denominator, $D$, is given by

$$
D=i+\delta t^{2}+\Gamma\left|E_{0}\right|^{2}+\gamma^{2}\left|E_{0}\right|^{4} / 4 .
$$

159 
It becomes obvious at this point that the details of the derivation are quite tedious and uninformative to the general reader. I shall relenate these details to Appendix $B$, and continue to develop the equations in terms of coefficients that appear in the appendix.

The polarization must be reduced to a form consistent with the slowly varying envelope approximation. In the form given in Eq. 20, $P_{0}$ varies substantially over ch. period of half a wavelength because of the interference between the counterpropagating pump beams. This variation is averaged out by integrating over a period to obtain a polarizability that is slowly varying. The details of this integration appear in Appendix B. The resulting slowly varying polarizability has two components, each of which is phase matched with one of the two pump waves (see Eq. 10 of Appendix B).

The propagation of the four waves in the medium is described by the wave equation. In this case, the wave equation includes the linear index of refraction, $n$, of the host, $K C 1$. Separating the zero- and first-order terms in the wave equation gives

$$
\nabla^{2} E_{0}+\frac{n^{2} v^{2}}{c^{2}} E_{0}=\frac{-4 \pi v^{2}}{c^{2} E_{s}} P_{0}
$$

and

(23b) $\quad \nabla^{2}(\Delta E)+\frac{n^{2} v^{2}}{c^{2}} \Delta E=\frac{-4 \pi v^{2}}{c^{2} E_{s}} \Delta P$. 
Using the expression for $P_{0}$ in the SVEA obtained in Eq. 10 of Appendix $B$ in Eq. 23 a givas the following:

(24a) $\frac{d \rho_{f}}{d z}=\frac{-\alpha_{0} \rho_{f}}{\cos (\theta)} I_{p 0}$
(24b) $\frac{d \rho_{b}}{d z}=\frac{\alpha_{0} \rho_{b}}{\cos (\theta)} I_{p 0}$

and

(24c) $\frac{d \phi+}{d z}=\frac{2 \alpha_{0} G}{\cos (\theta)}\left(\rho_{b}{ }^{2}-\rho_{f}{ }^{2}\right) I_{p} 2$.

The quantities $I_{p 0}\left(\rho_{f}, \rho_{b}\right)$ and $I_{p 2}\left(\rho_{f}, \rho_{b}\right)$ are

integrals related to the spatial averaging process and are defined in the appendix. These integrals depend only on the values of the pump wave amplitudes, and not the phases. Hence Eqs. (24a) and (24b) are independent of Eq. $(24 c)$.

A similar analysis is performed for the probe waves. The coupled equations for the probe waves are

$$
\frac{d E_{1}}{d z}=\alpha E_{1}+i k^{\star} E_{2} * \exp \left(i \phi_{+}\right)
$$

and

$(25 b)$

$$
\frac{d E_{2}}{d z}=-\alpha E_{2}-i k^{*} E_{1} * \exp \left(i \phi_{+}\right)
$$

or identical to the standard form, Eq. $5(\mathrm{c})$ and $5(\mathrm{~d})$ of Chapter IV. The coefficients $\alpha$ and $k$ are derived in Appendix B (see Eqs. 11-25). They involve complicated functions of the pump beam amplitudes, and are too cumbersome to repeat here. 
Any of the various solutions given in Chapter IV can now be applied. Pump attenuation can be ignored, and Abrams and Lind's solution, Eq. IV-7, used. The WKB approximate solution obtained by Rigrod, Eq. IV-10, could be applied at low intensity. Finally, the numerical technique that I developed in Eq. IV-12 to $17 \mathrm{can}$ be used. That is, Eqs. $23(a)$ and $23(b)$ can be numerically solved by an iterative process to obtain a solution for the pump beams consistent with the boundary conditions. The solution for the pump beam amplitudes can then be used in Eqs. $23(\mathrm{c})$ and 24 to propagate the probe waves and determine the reflectivity. This is the technique employed here, since samples with large attenuation and a large range of intensities are investigated. Solutions based on this model are presented and compared to experimental results in the next section.

\section{Experimental Results in $K C 1: K R e D 4$}

In this section the results of DFWM experiments in $\mathrm{KCl}: \mathrm{KReO}_{4}$ are presented and compared to the theory of the previous section. Experiments have been performed on resonance at the P-28 line of the $10-\mu \mathrm{m}$ band of the $\mathrm{CO}_{2}$ laser in two samples of different dopant density, and off resonance at $P-26$ and $P-30$ in the more heavily doped sample. The experiments on resonance demonstrate 
the need to include pump attenuation effects. The inadequacy of the two-level model in describing DFWM in $\mathrm{KCl}: \mathrm{KReO}_{4}$ is demonstrated in three of the four samples.

\section{On Resonance}

The experimental results on resonance are the first reported ${ }^{9}$ quantitative comparison of a resonant DFWM process with the theory of Abrams and Lind. ${ }^{2}$ Results for the weakly doped sample compare well with the Abrams and Lind model, because it is relatively transparent, and the effect of higher lying vibrational states is weak at the intensities employed in the experiment. The more heavily doped sample does not compare well with the Abrams and Lind model, because of the absorption of the pump beams by the medium, and because of effects of the higher vibrational states.

Results from the weakly doped sample are plotted in Fig. 5. A discussion of the experiment used to obtain these results is given in Chapter II. This 1-cm-long sanple had a measured small-signal transmission of 0.625 \pm 0.04 , corresponding to a field attenuation coefficient of $\alpha_{0}=0.235 \pm 0.030 \mathrm{~cm}^{-1}$. The curve is a fit of the three-level model from the previous section using $\alpha L=0.26$ and $\gamma=-.023$. The value for $\gamma$ is based on Ahrenkiel's spectroscopy and the 


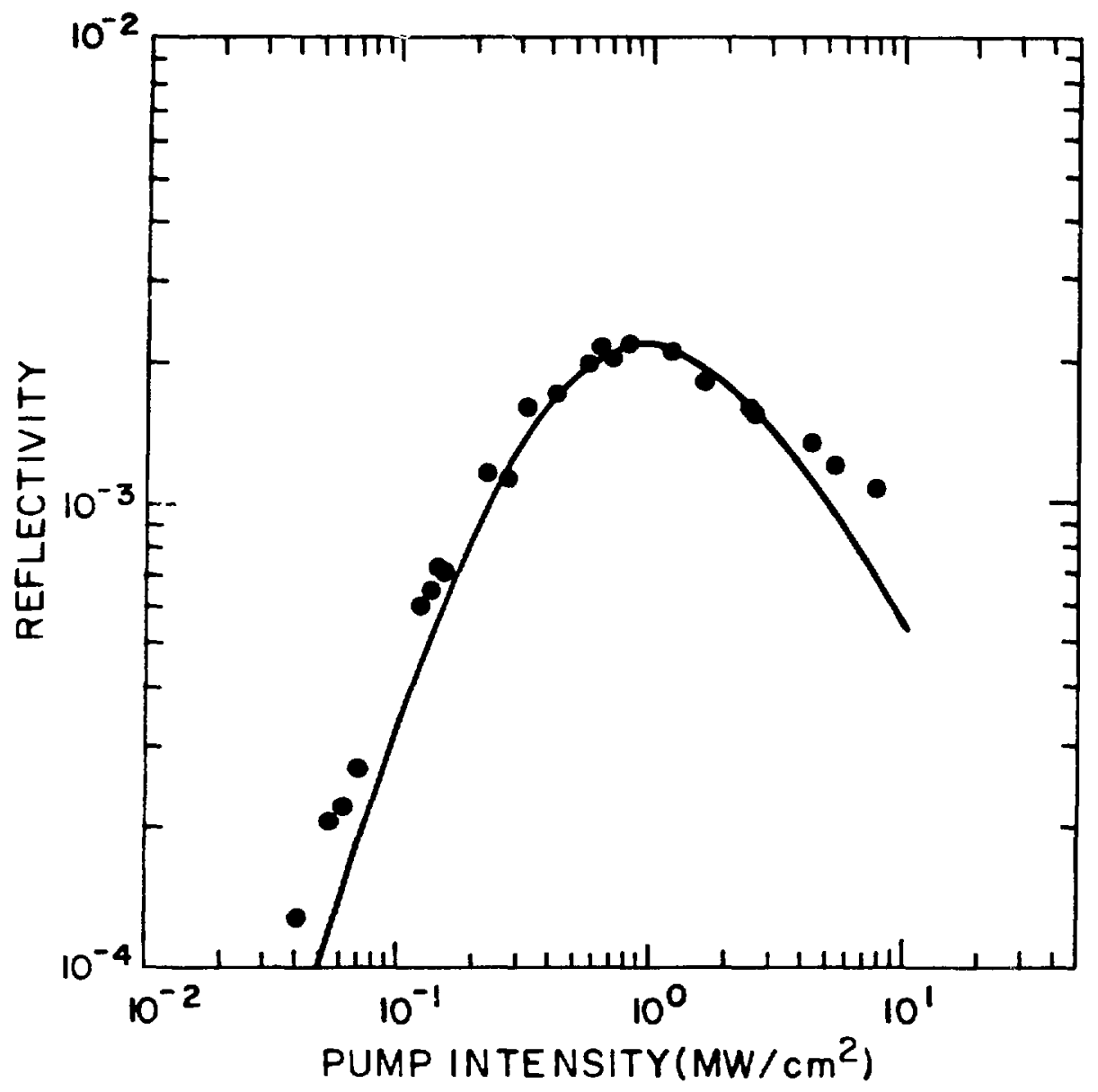

Figure VII-5. DFWM on resonance in a 1-cm sample of $\mathrm{KC}]: \mathrm{KReO}_{4}$. The small-signal field absorption coefficient is $\alpha_{0} L=0.235$. The curve is my three-level model with $\alpha_{0} L=0.26$ and $\gamma=-.023$. 
three-dimensional harmonic oscillator value for the ratio of the dipole moments, $\left|\mu_{12}\right|^{2} /\left|\mu_{0}\right|^{2}=4$.

This curve is essentially identical to the Abrams and Lind two-level model in this case. In fact this data has been cited as experimental justification of Abrams and Lind's two-level theory. ${ }^{9}$ It is obvious that more complicated models can give equivalent results under certain circumstances.

Figure 6 shows the results of DFWM experiments in a more heavily doped sample of $\mathrm{KCl}: \mathrm{KReO}_{4}$. In this sample the pump beams are severely attenuated by the medium. The small signal transmission of this sample is only $5 \%$, and the corresponding field attenuation coefficient is $\alpha_{0} L$ $=1.5$. The sample is $2-\mathrm{cm}$ long. In this case, the Abrams and Lind two-level model (solid curve) fails co correctly predict the reflectivity as a function of pump intensity by overestimating by more than a factor of 5 . It also fails to model the upward trend at high intensity. The first failure is due in large measure to their failure to consider the attenuation of the pump beams by the medium. This failure is a general feature of the technique that Abrams and Lind employed in obtaining their result. The second failure is slightly different, since a multilevel model rather than a two-level model is required to account for such features. 


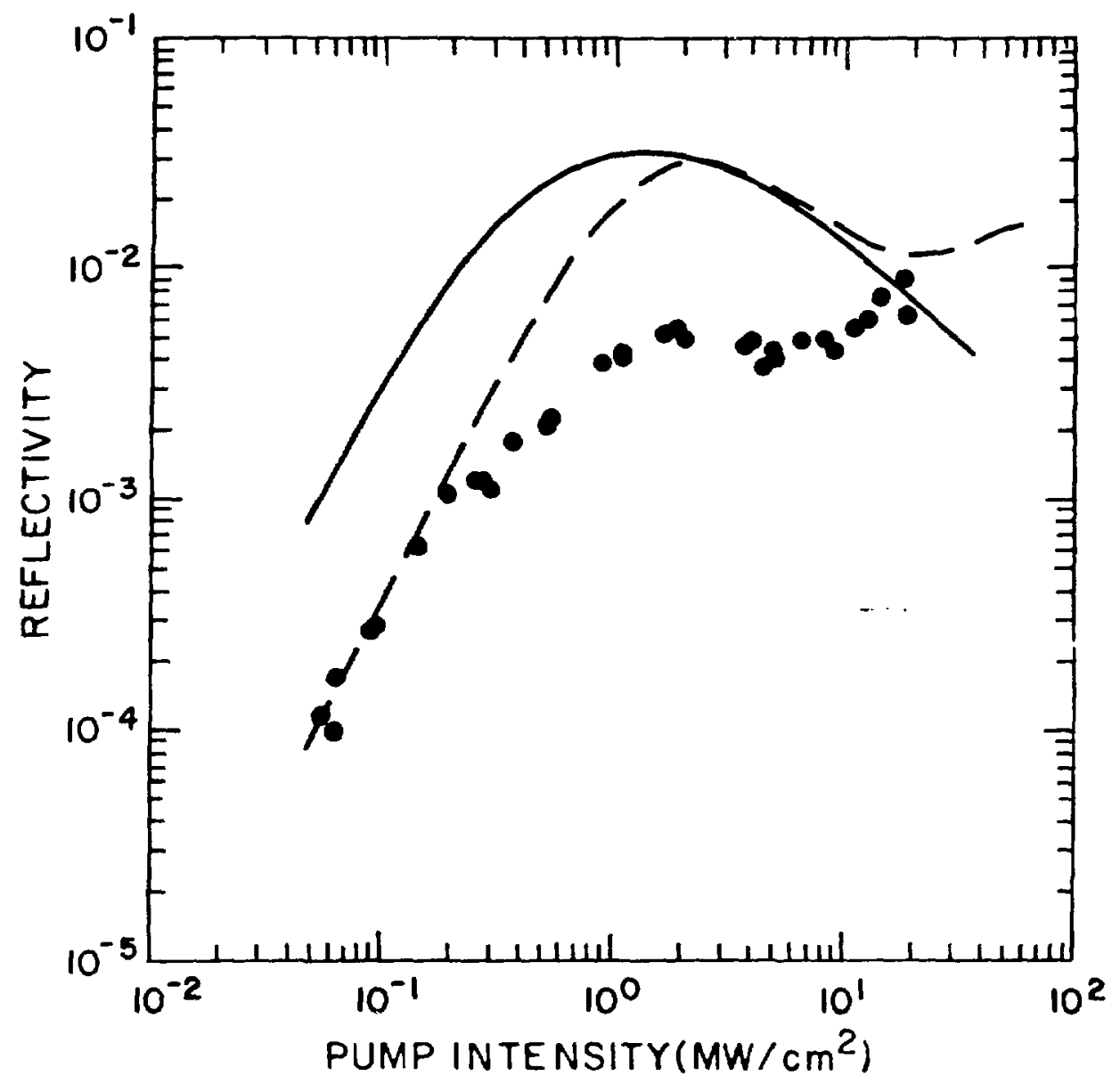

Figure VII-6. DFWM on resonance in a $2-\mathrm{cm}$ sample of $\mathrm{KC} 1: \mathrm{KReO}_{4}$. The small-signal absorption coefficient is $\alpha_{0} L=1.5$. The solid curve is Abrams and Lind's 2 two-level model. The dashed curve is my three-level model with $\gamma=-.08$. The discrepancies between the data and the model are not understood. 
The dashed curve is the three-level model of the previous section including pump attenuation and using the value $\gamma=-0.08$. This value has been chosen by roughly fitting the upward swing in reflectivity at high intensity. The large value for $\gamma$ used here is inconsistent with the expected value for $\gamma$ and with the results presented below. It is not inconsistent with the absorption experiments that have been performed and modeled with a two-level model.

This data is modeled qualitatively by the three-level theory. Quantitative agreement is lacking primarily at intermediate intensity and in the value of $\gamma$ used to fit the upward swing. If the expected value of $\gamma$ is used, the model is only slightly different from the dashed curve over most of the range. At high intensities, the model continues downward, with the upward swing occurring at much higher intensity.

\section{Off Resonance}

DFWM experiments have also been performed at $P-2 F$ and $\mathrm{P}-30$ of the $10-\mu \mathrm{m}$ band of the $\mathrm{CO}_{2}$ laser. These lias lie to the blue and red, respectively, of the resonance, and are approximately symmetric about the resonance. The normalized detunings from resonance, $\delta^{\prime}=\left(\nu-\omega_{10}\right) \mathrm{T}_{2}$, are $\delta^{\prime}(P-26)=4.05$ and $\delta^{\prime}(P-30)=-3.67$, about a $10 \%$ 
difference in magnitude. Since Abrams and Lind's model for the reflectivity depends only on the magnitude of $\delta '$, any differences in the experimental reflectivies is evidence for multilevel effects. I also note that, a) though the sample used in these experiments is the more heavily doped sample, the small-signal absorption of the sample is reduced by a factor of $1 /\left(1+\delta 1^{2}\right)$ by the decuning, or to about $7 \%$ of its value on resonance in the present case. Thus, the small-signal transmission of the more heavily doped sample this far off resonance is over $80 \%$. For such a weakly absorbing sample, the Abrams and Lind approximation of constant pump intensity is reasonable.

Experimental results on both these laser lines are plotted in Fig. 7. The curve is the Abrams and Lind model for $\delta^{\prime}=3.85$. Neither of the two sets of data is modeled well by this curve. There are also substantial differences between the two experimental results. These differences are due to multilevel effects.

Note that in Fig. 7 , both sets of data lie above the Abrams and Lind two-level model, whereas in Fig. 6, on resonance, the data lie below the two-level modei. There are two reasons for this. First, for the on-resonance data, the primary effect is absorptive in nature, so pump attenuation is severe, and second, the effect of the third 
level, which is reactive in nature, adds in quadrature to the absorptive effect of the resonant transition in DFWM. In contrast to this, for the off-resonance data of Fig. 7, absorption is substantially reduced, and both the effect of the third level and the effect of the two-level resonant transition are primarily reactive, so they add directly in the DFWM process.

Figure 8 shows experimental results for the $P-26$ 7ine. The curve is the three-level model using $\alpha_{0} L=$ 1.5 and $\gamma=-.016$. This value of $\gamma$ is based on Ahrenkiel's data for the spectroscopy of $\mathrm{KCl}_{\mathrm{K}} \mathrm{KReO}{ }_{4}{ }^{\text {T }}$ The fit to the data is reasonably good.

Ahrenkiel's data for the transition frequencies indicates that $\mathrm{P}-30$ is in two-photon resonance with $K C 1: K R e 0_{4}$, since $\left(\omega_{01}{ }^{+\omega_{12}}\right) / 2=934.8 \mathrm{~cm}^{-1}$ and $v(P-30)=934.895 \mathrm{~cm}^{-1}$. We also know that the width of the levels in $\mathrm{KCl}_{\mathrm{KReO}}$ is on the order of $1 \mathrm{~cm}^{-1}$. Although some two-photon absorption is expected, both theory and experiment would indicate that it has minimal effect.

An experiment has been performed at P-30 to look for two-photon absorption. No evidence has been found for two-photon absorption for intensities up to $150 \mathrm{MW} / \mathrm{cm}^{2}$ (or I/Is $=100)$. This result is supported by the three-level model presented here. 


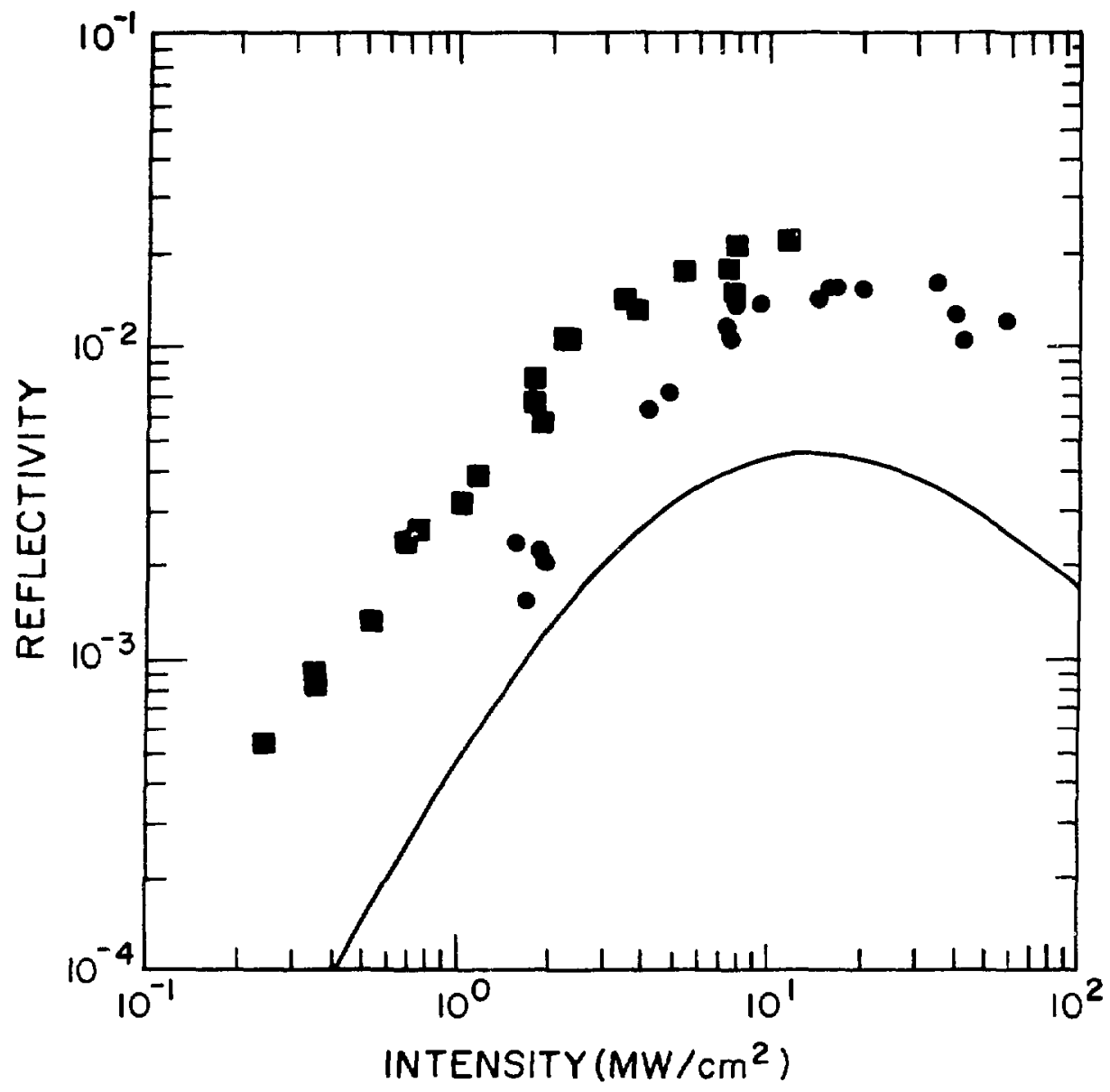

Figure VII-7. DFWM in the 2-cm cample of Fig. 6, detuned to the red (squares) and to the blue (circles) of
resonance. The solid curve is Abrams and Lind's? two-level model, with $\alpha_{0} L=1.5$ and $\delta^{\prime}=3.85$. Neither set of data is described well by the model. The two sets of data are also different from each other. These observations indicate that a two-level model is not adequate for describing DFWM in KCl:KReO4. 


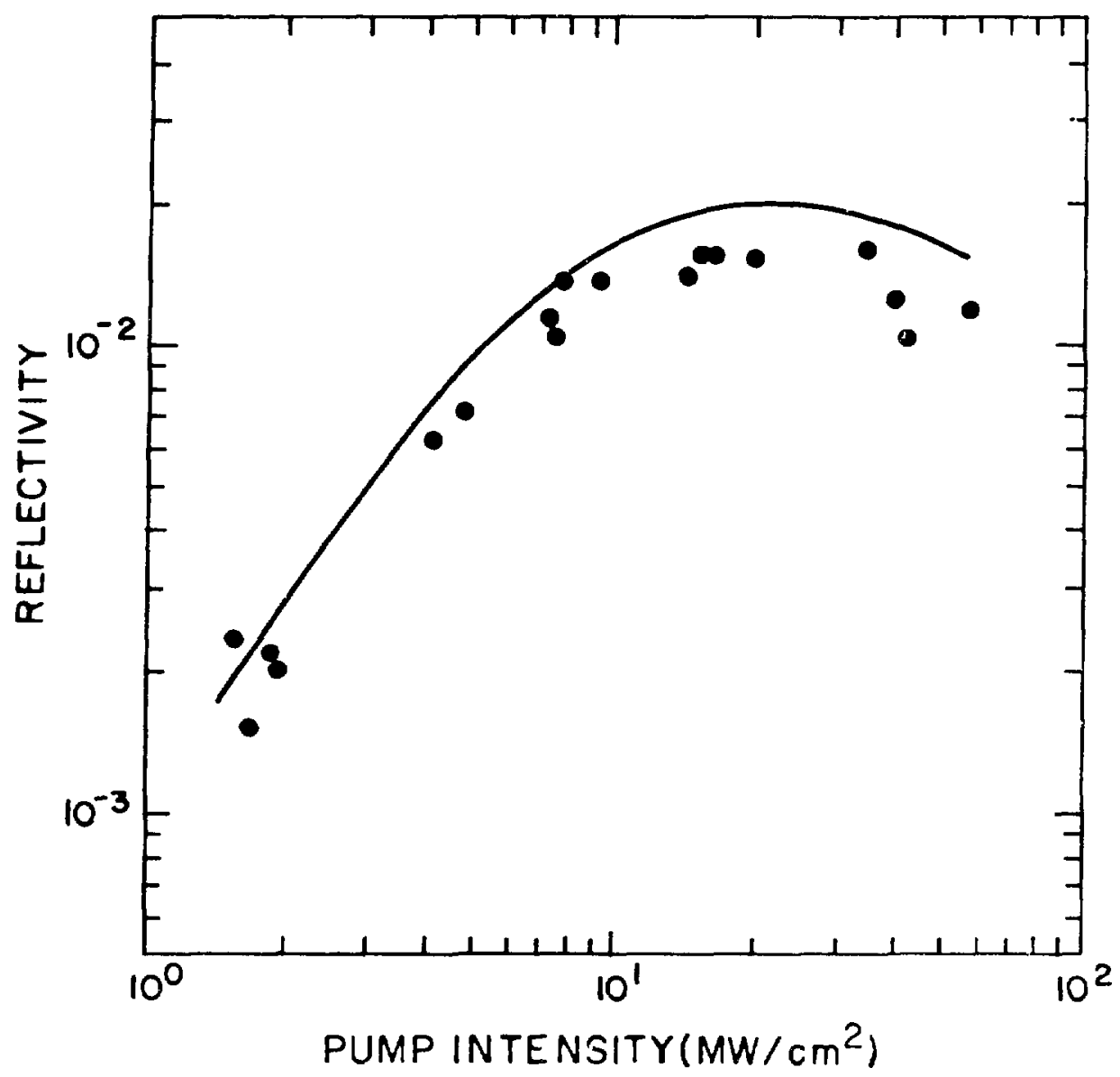

VII-8. DFWM in KCl:KReO4, detuned to the blue of resonance. In this case, the 1 aser is tuned to $P-26$ of the $10-\mu \mathrm{m}$ band of the $\mathrm{CO}_{2}$ laser. The curve is my three-level model with $\alpha_{0} L=1.5, \delta^{\prime}=4.05$ and $\gamma=-0.016$. These values are based cn Ahrenkiel's work and the harmonic oscillator value for the ratio of the dipole moments. 
The population of the highest state is

$$
N_{2}=N \gamma \frac{\omega_{2} 1}{T_{2}\left(\omega_{2} 1^{2}-v^{2}\right)} \frac{|E|^{4} / E_{s}{ }^{4}}{\left(1+\delta^{2}+|E|^{2} / E_{s}^{2}\right)} .
$$

where $\delta$ is the full normalized detuning of Eq. 10 and $N$ is the number density of absorbers. From this expression, I find that only $2.3 \%$ of the population is in the third levei at $|E|^{2} / E_{S}^{2}=10$. Assuming the upper state relaxes in a time $T_{1}$, the intensity absorbed is approximately $I_{a}=N_{2} 2 n v L / T_{1}$, where $L$ is the sample length. For $\mathrm{N}=3.3 \times 10^{16}$ (as in the more heavily doped sample) and $|E|^{2} / E_{S}^{?}=10$, this gives $I_{\partial}=$ $2.43 \times 10^{5} \mathrm{~W} / \mathrm{cm}^{2}$, or an absorption of about $3.2 \%$ of the input intensity. For the intensities I have used in DFWM experiments, the population in the highest state is low even near a two-photon resonance, and one might expect the three-level model presented here to give reasonable results.

Results of the three-level model calculation are presented and compared to the data for P-30 in Fig. 9. This calculation is in better agreement with the data than the Abrams and Lind two-level model (see Fig. 7). There is still substantial disagreement at high intensity. The values used in the calculaton are $\delta^{\prime}=-3.67$ and $\gamma=-0.044$. A slightly lower value of $\gamma$ would improve the fit. The values used here are based on Ahrenkiel's measurements. 


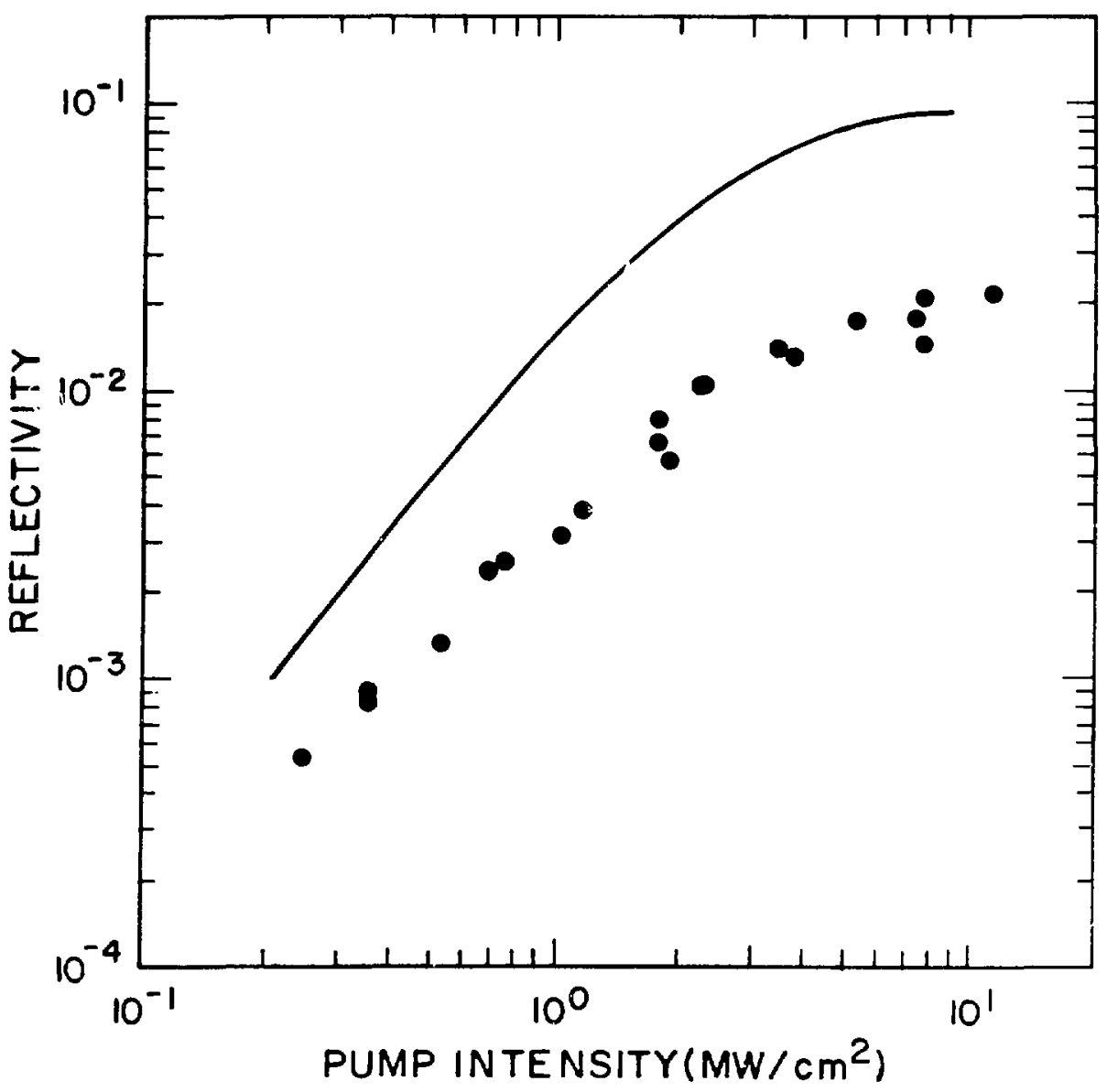

Figure VII-9. DFWM in KCl:KReO4, detuned to the red of resonance. In this case the laser is tuned to P-30 of the $10-\mu \mathrm{m}$ band of the $\mathrm{CO}_{2}$ laser. This is near a two-photon resonance. The curve is my three-level model with $\alpha_{0} L=1.5, \delta^{\prime}=-3.67$, and $\gamma=0.044$. These are expected values for $\delta '$ and $\gamma$. A smaller value of $\gamma$ would improve the fit. 
E. Discussion and Conclusions

In general the three-level model for the polarizability and the model of DFWM including pump attenuation effects have been relatively successful in explaining the experimental data in $\mathrm{KCl}: \mathrm{KReO}_{4}$.

on resonance, the reduction in reflectivity has been shown to be due in large measure to the attenuation of the pump beams by the medium. The three-level model has been shown to be capable of qualitatively explaining the increase in reflectivity observed at high intensity in the more heavily doped sample. This result is more qualitative than quantitative because the value of $r$ used to explain the result is about $3-i / 2$ times the expected value, and is also in disagreement with the results on resonance in the less highly doped sample and with the results off resonance in the same sample where the expectef values of $\gamma$ give reasonable results when compared to experiment.

In contrast to the results for $p$-type Ge in Chapter $V$, possible effects due to laser heating of the sample can be shown to be negligible in $\mathrm{KCl} \mathrm{KReO}_{4}$. First, I use Eq. 12 of Chapter $V$ to calculate the laser induced change in temperature. For $\mathrm{KCl},{ }^{13} \mathrm{c}=0.69 \mathrm{~J} / \mathrm{g}^{\circ} \mathrm{K}$ and $\rho=$ $1.99 \mathrm{~g} / \mathrm{cm}^{3}$. I find that $\Delta \mathrm{T}=.003^{\circ} \mathrm{K}$ when $\alpha_{I}=0.4$ $\mathrm{cm}^{-1}, I=10 \mathrm{MW} / \mathrm{cm}^{2}$, and $\tau=1 \mathrm{~ns}$. 
There are two heating effects in $\mathrm{KCl}: \mathrm{KRe} 0_{4}$, a change in the resonant frequency of the $v_{3}$ vibrational mode, and a thermal change in index of refraction. From the data in Fig. 2 and 4 , I find that $\Delta \omega_{12} / \Delta T=-0.01 / \mathrm{cm}^{\circ} \mathrm{K}$.

Thus the temperature induced change in resonant frequency is $-3 \times 10^{-5} \mathrm{~cm}^{-1}$, which is negligible compared to the full width at half maximum of $1 \mathrm{~cm}^{-1}$.

A thermally induced change in index of refraction cannot be the only mechanism for DFWM in this material because, as shown in Fig. 3, absorption of laser energy is a monotonic function of intensity, while from Fig. 6, the observed reflectivity is not monotonic. Furthermore, since ${ }^{13} \mathrm{dn} / \mathrm{dT}=-2.75 \times 10^{-5} /{ }^{\circ} \mathrm{K}$, the thermally induced -hange in index of refraction is $\Delta n k_{0}=-5 \times 10^{-4}$ $\mathrm{cm}^{-1}$, which is negligible compared to either the change in absorption coefficient, $\Delta \alpha \sim 1 \mathrm{~cm}^{-1}$, or the charge in index predicted by the three-level model, $\Delta n k_{0}$ 2 $0.5 \mathrm{~cm}^{-1}$. Thus neither of the two possible laser heating effects is significant.

The conclusions of this chapter are as follows: 1) The resonant DFWM data in the highly absorbing sample of $\mathrm{KCl}: \mathrm{KReO}_{4}$ forces one to use a model of DFWil which includes attenuation of the pump beams by the medium. And 2) Data taken off resonance in a strongly absorbing sample carringt be fit by the simple Abrams and Lind two-level 
model. This data, plus the data on resonance in a weakly absorbing sample, can all be explained by using a three-level model with parameters $\left(\delta^{\prime}, \gamma\right.$, and $\left.\omega_{12}\right)$ that are consonant with previous experiments or with simple theoretical models. 
1. R. K. Ahrenkiel, D. J. Dunlavy, and A. J. Sievers, "A new class of saturable absorbers at $10.6 \mu \mathrm{m}$ based on doped alkali halides, "IEEE Journal of Quantum Electronics QE-16, 225 (1980).

2. R. L. Abrams and R. C. Lind, "Degenerate four-wave mixing in absorbing media," Optics Letters 2, 94 (1978) and Erratum, Optics Letters 3 , 205 (7978).

3. See for example, G. Hertzberg, Molecular Spectra and Molecular Structure, I I Infrared and Raman Spectra of Polyatomic Molecules, (D. Van Nostrand Co., Princeton, 1945).

4. R. K. Ahrenkiel, J. F. Figueira, C. R. Phipps, D. J. Dunlavy, S. J. Thomas, and A. J. Seivers, "A new saturable absorber for the $\mathrm{CO}_{2}$ laser using doped KC1," Applied Physics Letters 33, 705 (1978).

5. Cooling shifts the absorption resonance to the blue. This data is taken at $105^{\circ} \mathrm{K}$ (see Ref. 1).

6. See, for example, A. Yariv, Quantum Electronics (John Wiley and Sons, Inc. New York, 1975), p. 149.

7. L. M. Narducci, W. W. Eidson, P. Furcinitti, and D. C. Eteson, "Theory of a two-photon laser amplifier," Physical Review A 16, 1665 (1977).

8. D. C. Haueisen, "Doppler-free two-photon spectroscopy using degenerate four-wave mixing," Optics Communications 28, 183 (1979).

9. T. Fu and M. Sargent III, "Theory of two-photon phase conjugation," Optics Letters 5,433 (1980).

10. D. G. Steel and J. F. Lam, "Two-photon coherent-transient measurement of the nonradiative collisionless dephasing rate in $\mathrm{SF}_{6}$ via Doppler-free degenerate four-wave mixing, "Physical Review Letters $\underline{43}, 1588(1979)$.

i1. D. G. Steel, R. C. Lind, and J. F. Lam, "Degenerate four-wave mixing in a resonant homogeneously broadened system," Physical Review A 23, 2513 (1981). 
12. D. E. Watkins, J. F. Figueira, and S. J. Thomas, "observation of resonantly enhanced degenerate four-wave mixing in doped alkali halides," Optics Letters 5,169 (1980).

13. C. S. Sahagian and C. A. Pitha, Compendium on High Power Infrared Laser Window Materials, (Air Force Cambridge Research Laboratories, f.FCRL-72-0170, Bedford, MA 1972) p. 42 . 


\section{CHAPTER VIII}

\section{CONCLUSION}

A. Review

In this thesis, I have presented theoretical and experimental studies of degenerate four-wave mixing by three different mechanisms. These are the nonlinear index of refraction of a lossless, kerr-like material, the saturable absorption of a resonant optical transition, and the formation of an optically induced free-carrier grating.

DFWM in a lossless, Kerr-like medium has been examined in both the small-signal and large-signal limits. In the small-signal limit, z time-independent theory has been shown to agree with experimental data in Ge for samples with optical path length less than $20 \%$ of the laser pulse length. For longer Ge samples, the time-independent theory is invalid; for example, it has been shown that the nonlinear susceptibility of Ge deduced from these samples is as much as a factor-of-three below the value carefully established earlier by ellipse rotation measurements.

For DFWM in Ge in the large-signal limit, the mean absolute deviation of the experimental data from a theoretical model with no free parameters is about $8 \%$. This deviation can be accounted for by variations in the approximately constant intensity of the pump beams. 
Theoretical models for DFWM in saturable absorbers also have been derived. The novel feature of these models is that pump beam attenuation by the medium has been included. This has been shown to be extremely important in media with substantial loss. For example, in a medium with a small-signal attenuation coefficient of 6 , the mode? including pump attenuation predicts a DFWM reflectivity three orders of magnitude less than a model which ignores pump attenuation.

P-type Ge has been used to study the inixture of Kerr-like and saturable absorption effects in DFWM. To treat p-type Ge, it has been necessary to generalize existing theory to the case of a broadband, inhomogeneously broadened medium. Experimental results in a lightly doped, $6-\mathrm{mm}$ sample of $p$-type Ge agree with the theoretical model. Below a pump intensity of $10 \mathrm{MW} / \mathrm{cm}^{2}$, the saturable absorption dominates the DFWM process. Between 10 and $100 \mathrm{MW} / \mathrm{cm}^{2}$, the Kerr-like mechanism dominates. These two mechanisms are accounted for in the model. Above $100 \mathrm{MW} / \mathrm{cm}^{2}$, optically induced free-carrier generation occurs, and this mechanism dominates. As a general statement, for p-type Ge samples with smali-signal transmission of less than about $20 \%$, the model and the experimental results diverge noticeably. Free carriers can be induced in Ge by a cascade 
process involving several $\mathrm{CO}_{2}$-laser photons. This free-carrier generation process can be used as a mechanism for DFWM and amplified reflection by this process has been observed. The reflectivity is observed to depend on the 11 th power of the pump intensity in this regime. Both the magnitude and the intensity dependence of the reflectivity have been successfully modeled by calculating the change in index of refraction due to the generation of free carriers.

Finally, results of DFWM experiments in the doped alkali halide $\mathrm{KCl}: \mathrm{KReO}_{4}$ have been presented. $\mathrm{KCl}: \mathrm{KReO}_{4}$ is the only known homogeneously broadened medium at $\mathrm{CO}_{2}-1$ aser wavelengths. The DFWM results in this material were the first to illustrate the importance of pump attenuation effects in models for DFWM in saturable absorbers.

Additionally, it has been shown that although the Sazurable absorption in $\mathrm{Kil} \mathrm{KReO}_{4}$ is described by a two-level model, a three-level model is needed to predict the DFWM response of this material. There are two features of the experimental results in $\mathrm{KCl} \mathrm{KReO}_{4}$ that require a three-level model for their explanation. These are the increased reflectivity at high pump intensity where the two-level model predicts a decrease, and the frequency-asymmetric behavior of the observed reflectivity 
as the laser is tuned through resonance with the ground state transition. A model has been developed where the third level acts as a perturbation on the lower two levels. These features have been qualitatively modeled by the three-level model.

B. Directions for future research

Throughout the thesis I have studied only those aspects of DFWM related to the magnitude of the reflectivity. Although my experiment detects only waves counterpropagating to the input probe wave, and hence of appropriate wave vector for phase conjugation, no attempt has been made to determine the extent to which the reflected wave is the phase conjugate of the input signal wave. Some si h measurements have been made by others, 1,2 but there has not been a thorough quantitative study.

For example, the simplest constraints on phase conjugation via DFWM are geometric. These can be understood through the holographic analogy and the resolution of the grating involved in the DFWM process. More complicated constraints arise from the nonlinear nature of the interaction. I have shown that the magnitude of the reflectivity in a Kerr-like medium, Ge, is reduced by the effect of pump depletion. The phase conjugate nature of such a reflection is undoubtedly also 
affected by pump depletion because of the nonlinear dependence of the interaction on the input signal wave.

Using a Kerr-like medium for DFWM may also result in self focusing of the pump beams. This may ilave a deleterious effect on phase conjugation long before it affects the reflectivity. A similar problem can arise in saturable absorbers because of apodization of the pump beams by the medium.

Further studies of DFWM in the plasma-formation regime in Ge could prove fruitful if substantially shorter laser pulses are used. This would avoid the problem of laser-induced surface damage of the Ge, but retair the possibility of very large reflections. Again, the question of quality of the phase conjugate wave must be addressed, although similar work in si indicates that the quality may be quite good.'

Further experimental work is also needed to characterize possible nonlinear index of refraction mechanisms in p-type Ge. Most significant is the nonlinear index associated with the resonant transition tinat has been predicted by James. ${ }^{3}$ Developing experimental techniques that can distinguish the various mechanisms for nonlinear refraction is in itself a challenge. 


\section{Footnotes}

1. J. P. Woerdman, "Formation of a transient free-carrier hologram in Si," Optics Communications ?, 212 (1971).

2. E. Cergmann, I. Bigio, B. Feldman and R. Fisher, "High-efficiency pulsed $10.6-\mu \mathrm{m}$ phase conjugate reflection via degenerate four-wave mixing," Optics Letters $3,82(1978)$.

3. R. B. James and D. L. Smith, "Laser-induced changes in he dispersive properties of $p-G e$ due to intervalence-band transitions," Physical Review B 23, 4044 (1981). 


\section{ACKNOWLEDGEMENTS}

My deepest appreciation is extended to my supervisor at Los Alamos, Claude Phipps. His guidance and experience have been invaluable to me in my research.

I am greatly indebted to Bill Rigrod and Don Haueisen for many interesting and informative technical discussions.

I would like to thank Norval Fortson and John Rehr for their concera ind encouragement.

I am a so grateful to Joe Figueira for his support as Group Leader, and to Scott Thomas and Bob Harrison for their techilical assistance in performing experiments.

The excellent job of typing the manuscript was done by Sylvia Charmatz.

Finally, I wish to dedicate this work to my wife, Maxine. 


\section{B IBL IOGRAPHY}

1. R. L. Abrams and R. C. Lind, "Degenerate four-wave mixing in absorbing media," Optics Letters 2, 94 (1978) and Erratum, Optics Letters $\underline{3}, 205$ (T9́78).

2. R. K. Ahrenkiel, D. J. Dunlavy, and A. J. Sievers, "A new class of saturable absorbers at $10.6 \mu \mathrm{m}$ based on doped alkali halides," IEEE Journal of Quantum Electronics QE-16, 225 (1980).

3. R. K. Ahrenkiel, J. F. Figueira, C. R. Phipps, D. J. Dunlavy, S. J. Thomas, and A. J. Seivers, "A new saturable absorber for the $\mathrm{CO}_{2}$ laser using doped KC1," Applied Physics Letters 33, 705 (1978).

4. Yu. A. Anan'ev, and A. I. Urbanovich, "Mechanism of diffraction of light by optically induced gratings in absorbing media," Soviet Journal of Quantum Electronics $\underline{5}, 1320(1976)$.

5. E. Bergmann, I. Bigio, B. Feldman, and R. Fisher, "High-efficiency pulsed 10.6-um phase conjugate reflection via degenerate four-wave mixing, "Optics Letters $\underline{3}, 82$ (1978).

6. R. R. Birss, "Property tensors in magnetic crystal classes," Proceedings of the Physical Society 79,946 (1962).

7. N. B Toembergen, Nonlinear Optics (W. A. Benjamin, Inc., New York, 1965).

8. D. M. B $100 \mathrm{~m}$, P. F. Liao, and N. P. Economou, "Observation of amplified degenerate four-wave mixing in atomic sodium vapor." Optics Letters 2, 58 (1978).

9. H. B. Briggs and R. C. Fletcher, "Absorption of infrared light by free carriers in germanium," Physical Review 91, 1342 (1953).

10. E. Burstein, et al., "Optical and Photoconductive properties of silicon and germanium, " in Photoconductivity Conference 1954 (Wiley, New York, 1954).

11. E. D. Capron and 0. L. Brill, "Absorption Coefficient as a function of resistance for optical germanium at $10.6 \mu \mathrm{m}, "$ Applied Optics 12, 569 (1973). 
12. M. Cardona, W. Paul, and H. Brooks, "Dielectric constant of germanium and silicon as a function of volume, "Journal of the Physics and Chemistry of Solids $\underline{8},, 204$ (1959).

13. Y. Danileiko, A. Epifanov, T, Lebedeva, A. Manenkov, V. Milyaev, and S. Sidorin, Reprint No. 10,

P. N. Lebedev, Physical Institute, Moscow (1977).

14. H. J. Eichler, "Laser induced grating phenomena," Optica Acta 24, 031 (1977).

15. H. M. A. El Sum, Reconstruction Wavefront Microscopy, (Ph.D. thesis, Stanford University, 1952).

16. A. Elci, D. Rogovin, D. Depatie, and D. Haueisen, "Phase conjugation in ammonia," Journal of the Optical Society of America 70,990 (1980).

17. J. Feinberg and R. W. Hellwarth, "Phase-conjugating mirror with continuous-wave gain," Optics Letters $\underline{5}$, $519(1980)$.

18. J. F. Figueira, S. J. Czuchlewski, C. R. Phipps, Jr., and S.J. Thomas, "Plasma-breakdown retropulse isolators for the infrared," Applied Optics 20,838 (1981).

19. R. A. Fisher and B. J. Feldman, "On-resonant phase-conjugate reflection and amplification at $10.6 \mathrm{\mu m}$ in inverted $\mathrm{CO}_{2}$," Optics Letters 4 , 140 (1979).

20. P. Flubacher, A. J. Leadbetter, and J. A. Morrison, "The heat capacity of pure silicon and germanium and properties of their vibrational frequency spectrum," Philosophical Magazine 4,273 (1959).

21. C. Flytzanis, "Third-order optical susceptibilities in IV-IV and III-V semiconductors," Physics Letters $31 \mathrm{~A}$, $273(1970)$.

22. T. Fu and M. Sargent III, "Theory of two-photon phase conjugation," Optics Letters $\underline{5}, 433$ (1980).

23. D. Gabor, "Microscopy by reconstructed wave-fronts," Proceedings of the Royal Society, Series A, 197, 454 $(1949)$.

24. H. J. Gerritsen, "Nonlinear effects in image formation," Applied Physics Letters 10, 237 (1967). 
25. Gmelins Handbook of Inorganic Chemistry, 8th Edition, "Germanium," Weinheim, Verlag Chemie, GmbH 1958.

26. D. Grischkowsky, N. S. Shiren, and R. J. Bennett, "Generation of time reversed wave fronts using a resonantly enhanced electric nonlinearity," Applied Physics Letters 33,805 (1978).

27. D. S. Hamilton, D. Heiman, J. Feinberg, and R. W. Hellwarth, "Spatial diffusion measurements in impurity doped solids by degenerate four-wave mixing," Optics Letters 4,124 (1979).

28. D. C. Haueisen, "Doppler-free two-photon spectroscopy using degenerate four-wave mixing, "Optics Communications 23 , 183 (1979).

29. R. W. Hellwarth, "Generation of time-reversed wavefronts by non inear refraction, "Journal of the optical Society of America 67, 1 (1977).

30. G. Hertzberg, Molecular Spectra and Molecular Structure, II Infrared and Raman Spectra of Polyatomic Molecules, (D. Van Nostrand Co., Princeton, 1945).

31. H. Hsu, "Large-signal theory of phase-conjugate backscatterings, "Applied Physics Letters 34,855 (1979).

32. E. V. Ivakin, I. P. Petrovich, and A. S. Rubinov, "Self diffraction of radiation by light induced phase gratings," Soviet Journal of Quantum Electronics 3 , 52 (1973).

33. E. V. Ivakin, I. P. Petrovich, A. S. Rubinov, and B. I. Stepanov, "Dynamic holograms in an amplifying medium," Soviet Journal of Quantum Electronics $\underline{5}$, 840 $(1975)$.

34. R. K. Jain and M. B. Klein, "Degenerate four-wave mixing near the band gap of semiconductors, "Applied Physics Letters 35,454 (1979).

35. R. K. Jain, M. B. Klein, and R. C. Lind, "High-efficiency degenerate four-wave mixing of 1.06 $\mu m$ radiation in silicon, "Optics Letters 4,328 $(1979)$.

36. R. K. Jain and D. G. Steel, "Degenerate four-wave mixing of $10.6 \mu \mathrm{m}$ radiation in $\mathrm{Hg} 1-x \mathrm{Cd}_{x} \mathrm{Te}, "$ Applied Physics Letters 37, 1 (1980). 
37. R. B. James and D. L. Smith, "Theory of nonlinear infrared absorption in p-type germanium, "Physical Review Letters 42, 1495 (1979).

38. R. B. James and D. L. Smith, "Laser-induced changes in the dispersive properties of $p-G e$ due to intervalence-band transitions," Physical Review B 23 , 4044 (1981).

39. S. Jha and N. Bloembergen, "Nonlinear optical susceptibilities in group-IV and III-V semiconductors," Physical Review 171, 891 (1968).

40. A. H. Kahn, "Theory of infrared absorption of carriers in germanium and silicon," Physical Review 97, 164? $(1955)$.

41. Keilmann, "Infrared saturation spectroscopy in p-type germanium," IEEE Journal of Quantum Electronics, QE-12, $592(1976)$.

42. F. Keilmann and Jurgen Kuhl, "Broadband modulation of

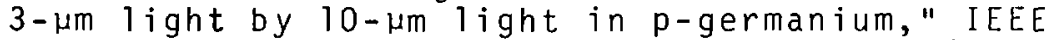
Journal of Quantum Electronics QE-14, 203 (1978).

43. M. A. Khan, P. W. Kruse and J. F. Ready, "Optical phase conjugation in $\mathrm{Hgl}-\mathrm{x} \mathrm{Cd}_{\mathrm{x}} \mathrm{Te}$, "Optics Letters 至, $261(1980)$.

44. Yu. I. Kruzhilin, "Self-adjusting laser-target system for laser fusion," Soviet Journal of Quantum Electronics $\underline{8}, 359(1978)$.

45. B. Lax and J. G. Mavroides, "Statistics and galvano-magnetic effects in germanium and silicon with warped energy surfaces," Physical Review 100, 1650 $(1955)$.

46. N. Lee, R. Aggarwal, and B. Lax, "Noncollinear phase-matched four-photon mixing of $\mathrm{CO}_{2} 1$ aser radiation in germanium," Optics Communications 19, 401 (1976).

47. E. M. Leith and J. Upatnieks, "Reconstructed wavefronts and communication theory, "Journal of the Optical Society of America 52, 1123 (1962).

48. M. D. Levenson and K. Chiang, "Application of phase conjugation to photolithography," presented at the Conference on Lasers and Electro-0ptics 181 (Washington, 0. C., 10.12 June 1987). 
49. P. F. Liao and D. M. Bloom, "Continuous-wave backward-wave generation by degenerate four-wave mixing in ruby," Optics Letters 3 , 4 (1978).

50. I. Liberman, "Application of phase conjugation to $\mathrm{CO}_{2}$ lasers," in Proceedings of the Los Alamos Conference on Optics 179, D. H. Liebenberg, Ed. (SPIE, Washington $1979), p .426$.

51. R. C. Lind, D. G. Stee 1, M. B. Klein, R. L. Abrams, C. R. Guiliano, and R. K. Jain, "Priase conjugation at $10.6 \mu \mathrm{m}$ by resonantly enhanced degenerate four-wave mixing," Applied Physics Letters 34, 457 (1979).

52. P. D. Maker and R. W. Terhune, "Study of optical effects due to an induced polarization third order in electric field strength," Physical Review 137, A801 $(1965)$.

53. J. H. Marburger, "Optical pulse integration and chirp reversal in degenerate four-wave mixing, "Applied Physics Letters 32,372 (1970).

54. J. H. Marburger and J. F. Lam, "Effect of nonlinear index changes on degenerate four-wave mixing, "Applied Physics Letters 35, 249 (1979).

55. J. H. Marburger and J. F. Lam, "Nonlinear theory of degenerate four-wave mixing, "Applied Physics Letters 34, $389(1979)$.

56. D. A. B. Miller, "rime reversal of optical pulses by four-wave mixing," Optics Letters 5 , 300 (1980).

57. D. A. D. Miller, R. G. Harrison, A. M. Johriston, C. T. Seaton, and S. D. Smith, "Degenerate four-wave mixing in InSb at $5^{\circ} \mathrm{K}$, "Optics Communications 32,478 $(1980)$.

58. D. A. B. Miller, S. D. Smith, and B. S. Wherrett, "The microscopic mechanism of third-order optical nonlinearity in InSb," Optics Communications 35, 221 (1980).

59. L. M. Narducci, W. W. Eidson, P. Furcinitti, and D. C. Eteson, "Theory of a two-photon laser amplifier," Physical Review A 16, 1665 (1977).

60. Yu. Ostrovskii, V. G. Sidorovich, D. I. Stastl'ko, and V. L. Tarrin, "Dynamic holngrams in sodium vapor," Soviet Technical Physics Letters 1, 442 (1975). 
61. A. Owyoung, "Ellipse rotation studies in laser host materials," IEEE Journal of Quantum Electronics QE-9, $1064(1973)$.

62. D. M. Pepper and R. L. Abrams, "Narrow optical bancipass filter via nearly degenerate four-wave mixing," Optics Letters $\underline{3}, 2$ (1979).

63. D. M. Pepper, J. Auyeung, D. Fekete, and A. Yariv, "Spatial convolution and correlation of optical fields via degenerate four-wave mixing," Optics Letters 3,7 $(1978)$.

64. C. Phipps, Jr., and S. Thomas, in Digest of Topical Meeting on Inertial Confinement and Fusion (Optical Society of America, Washington, D. C., 1980), paper TuB5.

65. C. R. Phipps, Jr., and S. J. Thomas, "Saturation behavior of p-type germanium at $\mathrm{CO}_{2}$ laser wavelengths," Optics Letters 1, 93 (1977).

66. C. Phipps, Jr. S. J. Thomas, and D. E. Watkins, "Effect of nonlinear refraction on beam brightness in laser fusion applications," in Proceedings of the International Conference on Lasers 179 (STS Press, Alexandria, Va., 1980), paper Q5.

67. R. J. Pressley, Ed., Handbook of Lasers, (CRC Press, Inc. Cleveland, 1977$)$.

68. W. W. Rigrod, R. A. Fisher, and B. J. Feldman, "Transient analysis of nearly degenerate four-wave mixing," Optics Letters $\underline{5}, 105$ (1980).

69. C. S. Sahagian and C. A. Pitha, Compendium on High Power Infrared Laser Window Materials, (Air Force Cambridge Research Laboratories, AFCRL-72-0170, Bedford, MA 1972) p. 42.

70. M. Sargent III, "Relaxation of hot holes in $p-G e, "$ Optics Communications 20, 298 (1977).

71. A. E. Siegman, "Dynamic interferometry and differential holography of irregular phase objects using phase conjugate reflection," Optics Communications 31 , 257 (1979). 
72. H. M. Smith, Principles of Holography (John Wiley and Sons, New York, 1975).

73. R. A. Smith, Semiconductors (Cambridge University Press, Cambridge, 1968).

74. 0. G. Steel and J. F. Lam, "Two-photon coherent-transient measurement of the nonradiative collisionless dephasing rate in $\mathrm{SF}_{6}$ via Doppler-free degenerate four-wave mixing," Physical Review Letters 43, 1588 (1979).

75. D. G. Steel and J. F. Lam, "Multiline phase conjugation in resonant materials," Optics Letters $\underline{5}$, $297(1980)$.

76. D. G. Steel, R. C. Lind, and J. F. Lam, "Degenerate four-wave mixing in a resonant homogeneously broadened system," Physical Review A 23, 2513 (1981).

77. B. I. Stepanov, E. V. Ivakin, and A. S. Rubinov, "on registration of plane and volume dynamic holograms in bleachable substances," Doklady Akad, Nank. SSSR 196, $567(1971)$.

78. A. Tomita, "Phase conjugation using gain saturation of a Nd:YAG iaser," Applied Physics Letters 34, 463 $(1979)$.

79. Mark Twain, Life on the Mississippi (Bantam Books, Inc., New York, 1972).

80. D. E. Watkins, J. F. Figueira, and S. J. Thomas, "Observation of resonantly enhanced degenerate four-wave mixing in doped alkali halides, "Optics Letters 5,169 (1980).

81. D. E. Watkins, C. R. Phipps, Jr., and S. J. Thomas, "Determination of the third-order nonlinear optical coefficients of Ge through ellipse rotation, "Optics Letters $\underline{5}, 248$ (1980).

82. D. E. Watkins, C. R. Phipps, Jr., and S. J. Thomas, "Observation of amplified reflection through degenerate four-wave mixing at $\mathrm{CO}_{2}$ laser wavelengths in germanium," Optics Letters 6,76 (1981). 
83. D. Weaire, B. S. Wherrett, D. A. B. Miller, and S. D. Smith, "Effect of low-power nonlinear refraction on laser-beam propagation in InSb, " Optics Letters 4, 331 (1979).

84. J. 0. White and A. Yariv, "Real-time image processing via four-wave mixing in a photorefractive medium," Applied Physics Letters 37, 5 (1980).

85. H. G. Winful, "Optical bistability in periodic structures and in degenerate four-wave mixing," in the Digest of Technical Papers, International Conference on Excited States and Multiresonant Nonlinear Optical Processes in Solids, D. S. Chemla, Ed. (Les Edition de Physique, Orsay, France, 1981), p. 55.

86. J. P. Woerdman, "Formation of a transient free-carrier hologram in Si," Optics Communications 2, 212 (1971).

87. J. P. Woerdman, "Some optical and electrical properties of a laser-generated free-carrier plasma in silicon," Philips Research Reports Supplements I, 1 (1971).

88. J. J. Wynne, "Optical third-order mixing in GaAs, Ge, Si, and InAs," Physical Review 178, 1295 (1969).

89. A. Yariv, Quantum Electronics (John Wiley and Sons, Inc. New York, 1975), p. 149.

90. A. Yariv and D. M. Pepper, "Amplified reflection, phase conjugation, and oscillation in degenerate four-wave mixing," Optics Letters 1, 16 (1977).

91. S. Y. Yuen, R. L. Aggarwal, and B. Lax, "Saturation of transmitted intensity of $\mathrm{CO}_{2}$ laser pulses in Ge," Journal of Applied Physics 5 I, 1146 (1980).

92. S. Y. Yuen, R. L. Aggarwal, N. Lee, and B. Lax, "Nonlinear absorption of $\mathrm{CO}_{2}$ laser radiation by nonequilibrium carriers in germanium, " Optics Communications 28 , 237 (1979).

93. P. Yu. Zel'dovich, V. I. Popovichev, V. V. Ragul'skii, and F. S. Faizullov, "Connection between the wavefronts of the reflected and exciting light in stimulated Mandel'shtam-Brillouin scattering, "Soviet Physics JETP 5,109 (1972). 
DETERMINATION OF THE THIRD-ORDER NONLINEAR OPTICAL COEFFICIENTS OF GERMANIUM THROUGH ELLIPSE ROTATION D. E. Watkins, C. R. Phipps, Jr., and S. J. Thomas Reprinted from Optics Letters $\underline{5}, 248$ (1980)

We describe the first reported direct determination of the $10-\mu m$ nonlinear susceptibility in intrinsic Ge through time-resolved ellipse rotation. We found that $x_{3}^{1111}=2.5 \times 10^{-11}$ esu, significantly below previous estimates.

The use of germanium in recent studies of phase conjugation ${ }^{1}$ and four-photon mixing ${ }^{2}$ has brought about renewed interest in its nonlinear optical properties. In this letter we present the results of the first reported direct determination of the nonlinear optical coefficients of Ge through time-resolved ellipse rotation. We review the principles of ellipse rotation and discuss the relationship of our results to those of other experiments.

When an intense, elliptically polarized electromagnetic wave is propagated through a nonlinear medium, the nonlinear change in the index of refraction is different for the left and the right circularly polarized components of the wave. This difference causes 
progressive rotation of the axes of the polarization ellipse.

This effect is due to the induced polarization which is third order in electric-field strength. For maierials with cubic symmetry, there are three independent components of the partially degenerate third-order nonlinear dielectric tensor, $x_{3}(-\omega, \omega, \omega,-\omega) .^{3}$ These are $x_{3}^{1111}, x_{3}^{1221}$, and $x_{3}^{1212}=x_{3}^{1122}$. If the only contribution to the nonlinearity is electronic in origin, the number of independent components is further reduced since $x_{3}^{1221}=x_{3}^{1212}$. These conditions hold for germanium in the $10-\mu m$ region. 4

owyoung ${ }^{5}$ has shown that the angle of ellipse rotation is directly related to these tensor components when an elliptically polarized plane wave propagates along a [100] crystal axis with the major axis of the ellipse oriented along a [100] or [110] axis in a transparent cubic medium. His result may be extended to include a nonzero absorption coefficient $\alpha$, giving for the rotation angle of the ellipse

$$
\begin{gathered}
\theta_{100}=\frac{-6 \pi \omega}{n c}\left(x_{3}^{1} 111-x_{3}^{1221}\right)\left[\left(1-e^{-\alpha L}\right) / \alpha\right] E_{o x} E_{o y}, \\
\theta_{110}=\frac{-12 \pi \omega}{n c}\left(x_{3}^{1221}\right)\left[\left(1-e^{-\alpha L}\right) / \alpha\right] E_{o x} E_{o y} .
\end{gathered}
$$


In Eq. (1), $w$ is the optical frequency, $n$ is the index of refraction, $c$ is the speed of light, and $L$ is the sample length. $E_{0 x}$ and $E_{o y}$ are the components of the elliptically polarized wave $\bar{E}=\left(E_{0 x} \hat{x}+i E_{o y} \hat{y}\right)$ $\exp [i(k z-\omega t)]$ in the medium at $z=0$. These are reduced by a factor of $\checkmark n=2$ relative to the external field components assuming that antireflection coatings are used.

To observe ellipse rotation, we used the arrangement shown in Fig. 1. The laser was a grating-controlled TEA $\mathrm{CO}_{2}$ oscillator-amplifier system, which provided up to 1 GW in a 2.3-nsec-duration pulse at $10.59 \mu \mathrm{m}$. We restricted our attention to a uniform central portion of the spatially filtered laser beam, using methods previously described. ${ }^{6}$ Calibrated attenuators controlled interisities in the experiment. A CdS retardation plate produced the correct polarization ellipticity for optimum sensitivity. ${ }^{5}$ Germanium samples could be oriented with crystal axes parallel to the ellipse axis within $1^{\circ}$, and rotation of the ellipse axis is no greater than $5^{\circ}$ were used to determine $x_{3}$. A multilayer dielectric polarization splitter reflected s polarization and passed p polarization to individual fast pyroelectric detectors. The splitter was oriented at $45^{\circ}$ relative to the incident ellipse axis for best sensitivity. No ellipse rotation signals could be 


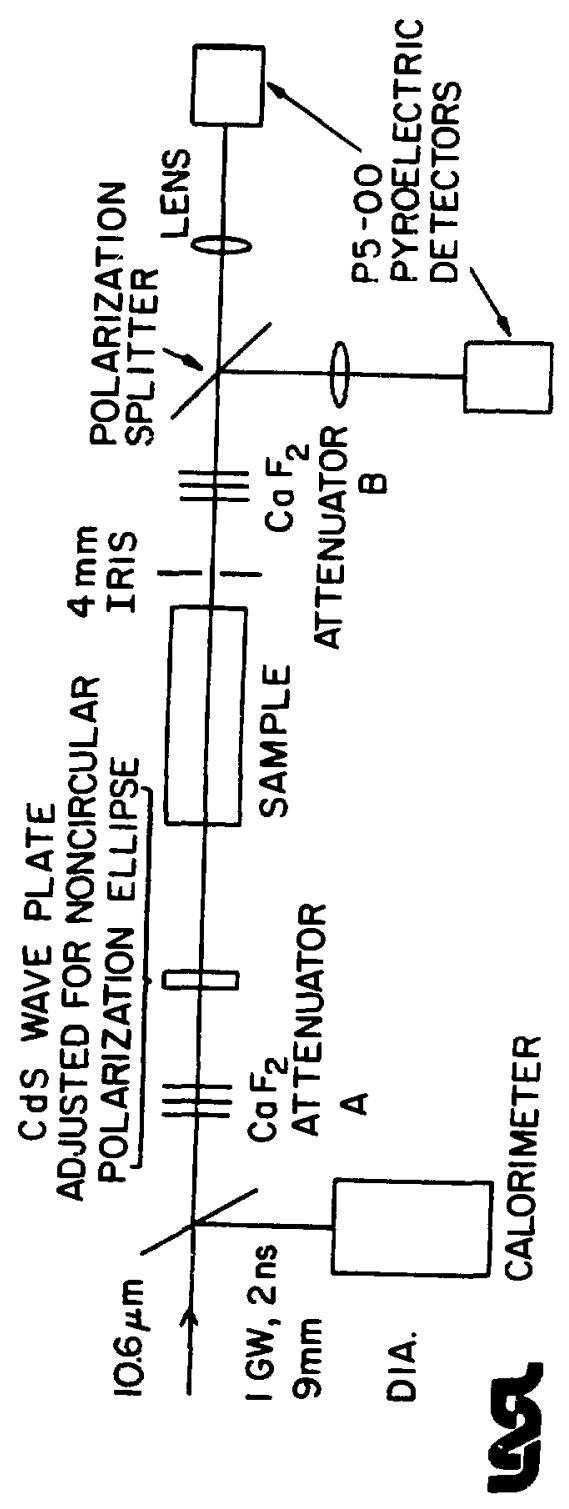

Figure A-1. Experimental arrangement for measurements of nonlinear susceptibility through ellipse rotation. 
detected in the absence of the nonlinear test sample.

The ratio of the peak intensities in the $s$ and $p$ polarizations is related to the angle of ellipse rotation (and hence to $x_{3}$ ) by the expression

$$
\frac{I_{s}}{I_{p}}=\frac{E_{o x^{2}} \cos ^{2}(\pi / 4-\theta)+E_{o y^{2}} \sin ^{2}(\pi / 4-\theta)}{E_{o x^{2}} \cos ^{2}(\pi / 4+\theta)+E_{o y^{2}} \sin ^{2}(\pi / 4+\theta)}
$$

This relationship is compared with data taken in $12 \mathrm{~cm}$ of single-crystal intrinsic germanium in Fig. 2. By fitting ti:e curves for both crystalline orientation to the data, it is possible to determine completely the third-order nonlinear dielectric tensor in this material.

The values for the third-order nonlinear susceptibility found in this study are $x_{3}^{1111}=$ $(2.5 \pm 0.3) \times 10^{-11}$ esu and $x_{3}^{1221}=(1.3 \pm 0.2) \times$ $10^{-11}$ esu. These values are a factor of 4 below those reported by wynne ${ }^{7}$ and currently quoted in the literature. ${ }^{2,8}$ our results are consistent with the theoretical calculations of Flytzanis, ${ }^{9}$ recent results in phase conjugation through degenerate four-wave mixing, results from similar phase-conjugation experiments performed by the authors, 10 and an independent measurement in which the on-axis brightness degradation that is due to whole-beam self-focusing in Ge was observed. $11,12,15$ In the last-mentioned experiment, 

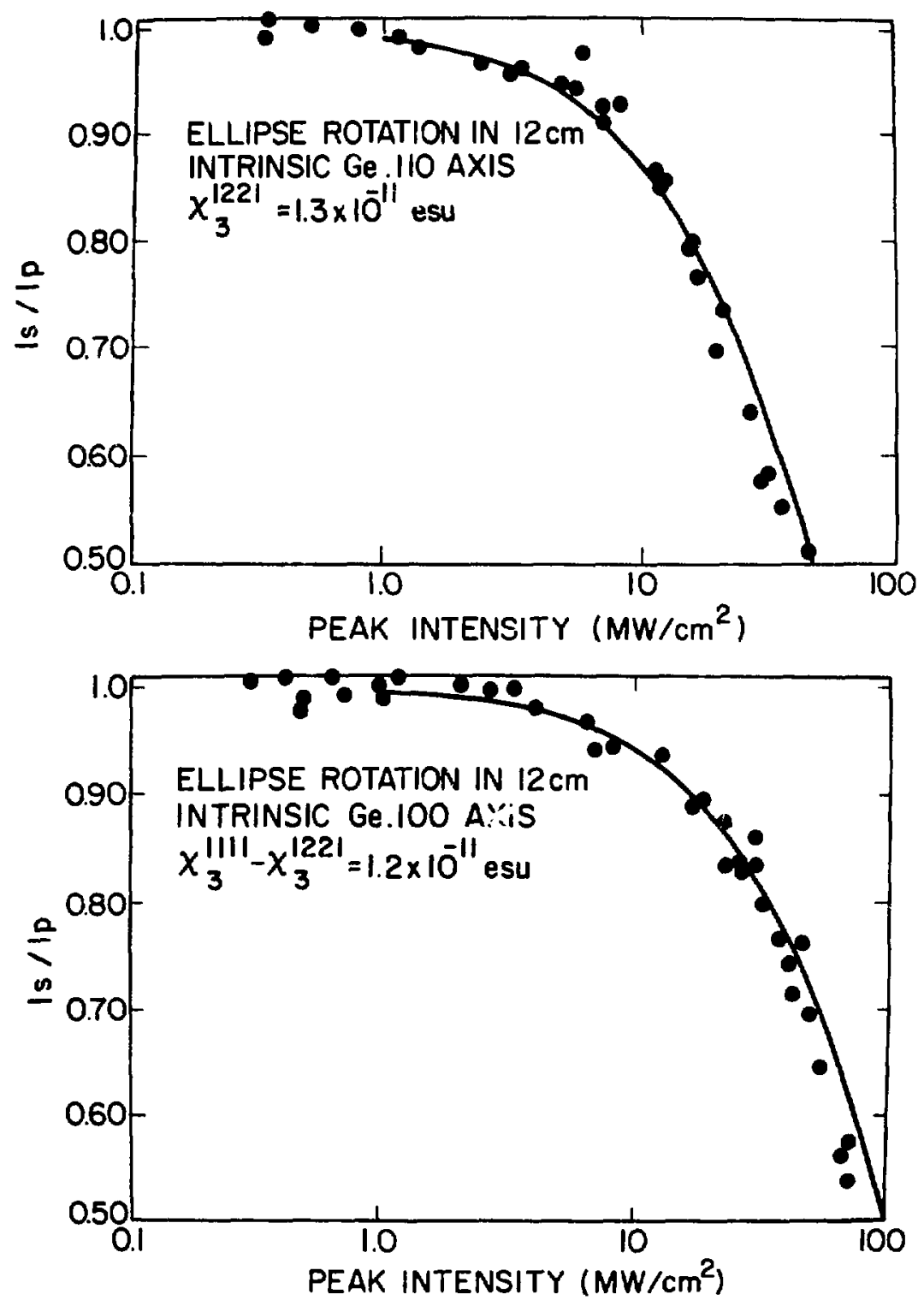

Figure A2. Ellipse rotation for two orientations in intrinsic germanium as discussed in the text. The ratio of the peak s-and p-polarized transmitted signals is plotted versus peak incident intensity for the incident optical field parallel to (top) a [110] axis and (bottom) a [100] axis in the material. The beam propagates along a [100] axis in either case. 
we obtained a value of $n_{2 \text { eff }}=3 \times 10^{-10}$ esu, which compares well with the value $n_{2}=(12 \pi / n) x_{3}^{1111}=2.4 x$ $10^{-10}$ esu calculated from the $x_{3}$ reported in this letter. Lind et $a 1 .{ }^{8}$ reported that theoretical predictions of the phase-conjugate reflectivity in Ge based on Wynne's value of $x_{3}$ were an order of magnitude above the reflectivities observed by Bergmann et al. 1 A calculation of $x_{3}$ can be made from the expression of the reflectivity given by Yariv and Pepper, ${ }^{13} R=\tan ^{2}(K L)$, where $L$ is the sample length, $K=(12 \pi \omega / n c) x_{3} E_{p}^{2}$, and $E_{p}$ is the amplitude of the pump beams inside the sample. Solving for $x_{3}$ gives

$$
x_{3}=\frac{n c \tan ^{-1} \sqrt{R}}{12 \pi \omega E_{p} L^{2}} .
$$

If the values reported by Bergmann et al, are used,' Eq. (3) gives $x_{3}=3.0 \times 10^{-11}$ esu, again in good agreement with $\mathrm{tl}$ - values we report here. Note that, in this case, a fac $r-o f-n=4$ error in the value for $x_{3}$ results when Eq. (3) is applied without properly distinguishing between internal and external pump-beam amplitudes.

Recent three-wave mixing experiments in Ge similar to those of Wynne have been reported by Lee et al. ${ }^{2}$ Values for $x_{3}$ calculated from this work range from factors of 2 thrcugh $g$ below the results given by Wynne, consistent with our factor of 4 . Part of the discrepancy in Ref. 2 
is due to the formation of electron-hole pairs in Ge at high intensities. 14,15 In our work, intensities were kept below the plasma-formation threshold. 15

We have presented values for the third-order nonlinear susceptibility of germanium at $10-\mu m$ wavelength that are a fartor of 4 below those previously reported. Careful consideration of related experimental results of various authors leads us to conclude that their results are consistent with the new values we report here rather than with those of Wynne. 


\section{References}

1. E. Bergmann, I. Bigio, B. Feldman, and R. Fisher, Opt. Lett. $\underline{3}, 82(1978)$.

2. N. Lee, R. Aggarwal, and B. I.ax, Opt. Commun. 9, 401 $(1976)$.

3. P. Maker and R. Terhune, Phys. Rev. 137A, 801 (1965).

4. S. Jha and N. Bloembergen, Phys. Rev. 171, 891 (1968).

5. A. Owyoung, IEEE J. Quãntum Electron. QE-9, 1064 (1973).

6. C. Phipps, Jr., and S. Thomas, Opt. lett. 1, 93 (1977).

7. J. Wynne, Phys. Rev. 178, 1295 (1969).

8. R. Lind, D. Steel, M. Klein, R. Abrams, C. Giuliano, and R. Jain, Appl. Phys. Lett. 34, 457 (1979).

9. C. Flytzanis, Phys. Lett. 31A, 273 (1970).

10. D. Watkins, C. Phipps, Jr., and S. Thomas, unpublished.

11. C. Phipps, Jr. D. Watkins, and S. Thomas, in Proceedings of the International Conference on Lasers 179 (STS Press, Alexandria, Va., 1980), paper Q5.

12. D. Weaire, B. Wherrett, D. Miller, and S. Smith, Opt. Commun. 27,133 (1978).

13. A. Yariv and D. Pepper, 0pt. Lett. I, 16 (1977).

14. S. Yuen, R. Aggarwa 7, N. Lee, and B. Lax, Opt. Commun. 28, $237(1979)$.

15. C. Phipps, Jr., and 3 . Thomas, in Digest of Topical Meeting on Inertial Confinement and Fusion (Optical Society of America, Washington, D. C., 1980), paper TuB5. 


\section{APPENDIX B}

THE THREE-LEVEL POLARIZABILITY IN THE SLOWLY VARYING ENVELOPE APPROXIMATION

\section{Introduction}

The counterpropagating pump beams in degenerate four-wave mixing create a standing wave. In a resonant medium, the response of the medium to this standing wave will be a spatial variation in the polarizability with a period of $1 / 2$ the optical wavelength. These rapid variations appear in the polarizability derived in section $B$ of Chapter VII. In order to remove the rapid variations and obtain a polarizability consistent with the slowly varying envelope approximation (SVEA), a spatial average over one period of the standing wave is taken.

In this appendix, the details of this spatial average are presented for both the zero- and first-order polarizability, Eqs. VII-20 and 21 . The coupling coefficients of Eqs. VII-25 are also derived.

\section{A. The zero-order polarizability in the SVEA}

The zero-order polarizability is given in Eq. VII-20. In order to average this polarizability, I use

(1)

$$
\left|E_{o}\right|^{2}=\rho_{f}^{2}+\rho_{b}{ }^{2}+2 \rho_{f} \rho_{b} \cos \phi_{p}
$$


where

$$
\phi_{p}=2 k \zeta+\phi_{f}-\phi_{b}
$$

Substituting this into Eq. VII-22, the denominator becomes

$$
D=A+B \cos \left(\phi_{p}\right)+C \cos ^{2}\left(\phi_{p}\right)
$$

where

$$
A=1+\delta 1^{2}+\Gamma\left(\rho_{f}{ }^{2}+c_{b}{ }^{2}\right)+\gamma^{2}\left(\rho_{f}{ }^{2}+\rho_{b}{ }^{2}\right)^{2} / 4
$$

$$
B=2 \Gamma \rho_{f} \rho_{b}+\gamma^{2} \rho_{f} \rho_{b}\left(\rho_{f}^{2}+\rho_{b}^{2}\right)
$$

and

$$
c=r^{2} \rho_{f}^{2} \rho_{b}^{2} .
$$

Similarly, the triple product in the numerator of Eq. VII-20 is most conveniently written

$$
\begin{aligned}
\left|E_{0}\right|^{2} E_{0}= & {\left[\rho_{f}{ }^{2}+\rho_{b}^{2}+2 \rho_{b}{ }^{2} \cos ^{2}\left(\phi_{p}\right)\right.} \\
& \left.-2 i \rho_{b}{ }^{2} \sin \left(\phi_{p}\right) \cos \left(\phi_{p}\right)\right] \rho_{f} \exp \left[i\left(k \zeta+\phi_{f}\right)\right] \\
+ & {\left[\rho_{f}^{2}+\rho_{b}^{2}+2 \rho_{f}^{2} \cos ^{2}\left(\phi_{p}\right)\right.} \\
& \left.+2 i \rho_{f}^{2} \sin \left(\phi_{p}\right) \cos \left(\phi_{p}\right)\right] \rho_{b} \exp \left[i\left(-k \zeta+\phi_{b}\right)\right] .
\end{aligned}
$$

The polarizability is integrated by substituting these results into Eq. VII-20 and integrating over the variable $\phi_{p}$ on an interval of $2 \pi$. Over this interval, the pump beam amplitudes are assumed to be constant, consistent with the SVEA. Note that since $\phi_{p}$ contains the 
quantity $\phi_{-}=\phi_{f}-\phi_{b}$, this variable is integrated away in this process.

In the case of the zero-order polarizability, three integrals must be obtained:

$$
I_{p 0}=\frac{1}{2 \pi} \quad \int_{-\pi}^{\pi} \frac{1}{D} d \phi_{p},
$$

$$
I_{p 2}=\frac{1}{2 \pi} \quad \int_{-\pi}^{\pi} \frac{\cos ^{2}\left(\phi_{p}\right)}{D} d \phi_{p},
$$

and

(7)

$$
I_{p}=\frac{1}{2 \pi} \quad \int_{-\pi}^{\pi} \frac{\sin \left(\phi_{p}\right) \cos \left(\phi_{p}\right)}{D} d \phi_{p} .
$$

The last integral is easily seen to vanish, since $\sin \left(\phi_{p}\right)$ is an odd function, and everything else in the integrand is even. The remaining two integrals have been evaluated for the case $B^{2}>4 A C$, with the results

$$
I_{p 0}=\frac{1}{A(\alpha-\beta)}\left[\frac{\alpha}{\sqrt{1-\alpha^{2}}}-\frac{\beta}{\sqrt{1-\beta^{2}}}\right]
$$

and

where

$$
I_{p 2}=\frac{1}{c}\left\{1+\frac{1}{(\alpha-\beta)}\left[\frac{\beta}{\sqrt{1-\alpha^{2}}}-\frac{\alpha}{\sqrt{1-\beta^{2}}}\right]\right\}
$$

$$
\alpha=\frac{B+\sqrt{B^{2}-4 A C}}{2 A} \text { and } B=\frac{B-\sqrt{B^{2}-4 A C}}{2 A} \text {. }
$$

Pulling everything together, the resulting slowly varying zero-order polarizability is 
(10) $P_{o}=\frac{n^{2} \alpha_{o} E_{s}}{2 \pi k}\left\{\left[i-\delta^{\prime}+G\left(\rho_{f}^{2}+\rho_{b}^{2}\right)\right] I_{p 0}\right.$

$$
\begin{array}{r}
\left.+2 G \rho_{b}^{2} I_{p 2}\right\} \rho_{f} \exp \left[i\left(k \zeta+\phi_{f}\right)\right] \\
+\frac{n^{2} \alpha_{o} E_{s}}{2 \pi k}\left\{\left[i-\delta^{\prime}+G\left(\rho_{f}^{2}+\rho_{b}{ }^{2}\right)\right] I_{p 0}\right. \\
\left.+2 G \rho_{f}^{2} I_{p 2}\right\} \rho_{b} \exp \left[i\left(k \zeta+\phi_{b}\right)\right] .
\end{array}
$$

This polarizability is used directiy in the text to obtain the pump equations, Eq. VII-24.

B. The first-order polarizability of the SVEA and the derivation of the coupling coefficients

In the first-order polarizability, phase matching the terms and identifying the coefficients $\alpha$ and $k$ is relatively simple. Phase matching is achieved by equating coefficients of the exponentials, $\exp \left( \pm i k \zeta_{l}\right)$. Since

$$
\Delta E=E_{1} \exp \left(i k \zeta_{1}\right)+E_{2} \exp \left(-i k \zeta_{1}\right)
$$

and

$$
\Delta E^{*}=E_{1} * \exp \left(-i k \zeta_{1}\right)+E_{2} * \exp \left(i k \zeta_{1}\right),
$$

it is obvious that the coefficients of $\Delta E$ become $\alpha$ and those of $\Delta E *$ become $K$. The factor in the polarizability $n^{2} E_{s} / 2 \pi k$, along with the factor $2 k$ on 
the left hand side of the wave equation in the SVEA, cancel the factor $4 \pi v^{2} / c^{2} E_{S}$ on the right hand side of the wave equation. There remains a factor of $i$, which is incarporated into $\alpha$, but appears explicitly in the cross-coupling term of the coupled equations, Eq. VII-25. I note that the imaginary part of $\alpha$ introduces a phase shift common to both waves, and therefore cannot effect the magnitude of the reflectivity (see Chapter IV, Eqs. 13 and 14). This factor does not appear in any solution given in Chapter IV for the coupled probe waves. The spatial averages that give the remaining terms are

$$
\alpha_{R}=\frac{-\alpha_{0}}{2 \pi \cos (\theta)} \int_{-\pi}^{\pi} \frac{1+\delta 1^{2}-\gamma^{2}\left|E_{0}\right|^{4 / 4}}{D^{2}} d \phi_{p}
$$

$$
\kappa_{I}=\frac{\alpha_{0} \exp \left(-i \phi_{+}\right)}{2 \pi \cos (\theta)} \quad \int_{-\pi}^{\pi}
$$$$
\frac{\left(\Gamma+\gamma^{2}\left|E_{0}\right|^{2} / 2\right) E_{0}^{2}}{D^{2}} d \phi_{p}
$$

and

$$
\begin{aligned}
K_{R} & =\frac{\alpha_{0} \exp \left(-j \phi_{+}\right)}{2 \pi \cos (\theta)}\left\{\int_{-\pi}^{\pi} \frac{\left[\delta^{\prime}\left(\Gamma+\gamma^{2}\left|E_{0}\right|^{2} / 2\right) E_{0}^{2}\right.}{D^{2}} d \phi_{p}\right. \\
& \left.+\int_{-\pi}^{\pi} \frac{G\left(1+\delta^{\prime 2}-\gamma^{2}\left|E_{0}\right|^{4 / 4)} E_{0}^{2}\right.}{D^{2}} d \phi_{p}\right\} .
\end{aligned}
$$

Although it is not readily apparent from Eqs. 12 and 13 , $K_{R}$ and $\kappa_{I}$ are both real (see Eq. 19). In the case of the probe waves, a more convenient variable for the 
integration is

$$
\phi_{p}{ }^{\prime}=\phi_{p} / 2=k \zeta+\left(\phi_{f}-\phi_{b}\right) / 2 .
$$

In terms of this variable,

$$
\left|E_{o}\right|^{2}=\left(\rho_{f}-\rho_{b}\right)^{2}+4 \rho_{f} o_{b} \cos ^{2}\left(\phi_{p}^{\prime}\right)
$$

and

where

$$
D=a+b \cos ^{2}\left(\phi_{p}^{\prime}\right)+c \cos ^{4}\left(\phi_{p}^{\prime}\right),
$$

$$
\begin{aligned}
& a=1+\delta 1^{2}+\Gamma\left(\rho_{f}-\rho_{b}\right)^{2}+\gamma^{2}\left(\rho_{f}-\rho_{b}\right)^{4} / 4, \\
& D=4 \Gamma \rho_{f} \rho_{b}+2 \gamma^{2} \rho_{f} \rho_{b}\left(\rho_{f}-\rho_{b}\right)^{2}, \\
& c=4 \gamma^{2} \rho_{f}^{2} \rho_{b}^{2} .
\end{aligned}
$$

and

The coupling coefficients are written in terms of the integrals

$$
I_{n}{ }^{\prime}=\frac{1}{\pi} \quad \int_{-\pi / 2}^{\pi / 2} \quad \frac{\cos ^{n^{\prime}}\left(\phi_{p}^{\prime}\right)}{D^{2}} d \phi_{p^{\prime}},
$$

where $n^{\prime}=0,2,4$, or 6 . These integrals are evaluated below. Of the coupling coefficients, $\alpha_{R}$ is the easiest to write in terms of these integrals:

$$
\begin{aligned}
\alpha_{R} & =\frac{-\alpha_{o}}{\cos (\theta)}\left\{\left[1+\delta \cdot 2-\gamma^{2}\left(\rho_{f}-\rho_{b}\right)^{4} / 4\right] I_{0}\right. \\
& \left.-2 \gamma^{2} \rho_{f} \rho_{b}\left(\rho_{f}-\rho_{b}\right)^{2} I_{2}-4 \gamma^{2} \rho_{f}^{2} \rho_{b}^{2} I_{4}\right\} .
\end{aligned}
$$

208 
To evaluate $\kappa$, I first need

$$
\begin{aligned}
E_{0}^{2}=\exp \left(i \phi_{+}\right)\left[-\left(\rho_{f}-\rho_{b}\right)^{2}+2\left(\rho_{f}{ }^{2}+\rho_{p}{ }^{2}\right) \cos ^{2}\left(\phi_{p}{ }^{\prime}\right)\right. \\
\left.+2 i\left(\rho_{f}{ }^{2}-\rho_{b}{ }^{2}\right) \sin \left(\phi_{p}^{\prime}\right) \cos \left(\phi_{p}^{\prime}\right)\right] .
\end{aligned}
$$

Note that the exponential term in this equation cancels the exponential of Els. 12 and 13 , and that the term in $\sin \left(\phi_{p}{ }^{\prime}\right)$ vanishes in the integral because it is odd. Substituting this into Ens. 12 and 13 , I find

$$
\begin{aligned}
\kappa_{I}=\frac{\alpha_{0}}{\cos (\theta)}\left\{-\left[\Gamma\left(\rho_{f}-\rho_{b}\right)^{2}+\gamma^{2}\left(\rho_{f}-\rho_{b}\right)^{4} / 2\right] I_{0}\right. \\
+\left[2 \Gamma\left(\rho_{f}{ }^{2}+\rho_{b}{ }^{2}\right)+\gamma 2\left(\rho_{f}-\rho_{b}\right) 4\right] I_{2} \\
\left.+4 \gamma^{2} \rho_{f} \rho_{b}\left(\rho_{f}{ }^{2}+\rho_{b}{ }^{2}\right) I_{4}\right\}
\end{aligned}
$$

and

where

$$
K_{R}=\frac{\alpha_{0}}{\cos (\bar{\theta})}\left(K_{R 0} I_{0}+K_{R 2} I_{2}+K_{R 4} I_{4}+K_{R 6} I_{6}\right)
$$

$$
\begin{aligned}
\kappa_{R 0}=\gamma^{2} G\left(\rho_{f}-\rho_{b}\right)^{6} / 4-\delta^{\prime} \gamma^{2}\left(\rho_{f}-\rho_{b}\right)^{4} / 2 \\
-\left[G\left(1+\delta^{2}\right)+\delta^{\prime} \Gamma\right]\left(\rho_{f}-\rho_{p}\right)^{2} \\
\kappa_{R 2}=2\left[G\left(1+\delta^{2}\right)+\delta^{\prime} \Gamma-\gamma^{2} G\left(\rho_{f}-\rho_{b}\right)^{4} / 2\right. \\
\left.+\gamma^{2}\left(\rho_{f}-\rho_{b}\right)^{2}\left(\delta^{\prime}+2 G \rho_{f} \rho_{b}\right)\right]\left(\rho_{f}^{2}+\rho_{b}^{2}\right)
\end{aligned}
$$

209 


$$
\begin{gathered}
-2 \gamma^{2}\left(\delta^{\prime}+2 G \rho_{f} \rho_{b}\right) \rho_{f} \rho_{b}\left(\rho_{f}-\rho_{b}\right)^{2} \\
K_{R 4}=4 \gamma^{2} \rho_{f} \rho_{b}\left[\delta^{\prime}-G\left(\rho_{f}-\rho_{b}\right)^{2}\right]\left(\rho_{f}{ }^{2}+\rho_{b}{ }^{2}\right) \\
+G \rho_{f} \rho_{b}\left(\rho_{f}-\rho_{b}\right)^{2}
\end{gathered}
$$

and

$$
\kappa_{R 6}=-8 \gamma^{2} G \rho_{f}^{2} \rho_{b}^{2}\left(\rho_{f}^{2}+\rho_{b}{ }^{2}, .\right.
$$

The integrals in Eq. 17 are evaluated by substituting $x=$ $\tan \left(\phi_{p}^{\prime}\right)$ to obtain

$$
\begin{aligned}
I_{2 n} & =\frac{1}{\pi} \int_{-\infty}^{\infty} \frac{\left(1+x^{2}\right)^{3-n}}{\left[a\left(1+x^{2}\right)^{2}+b\left(1+x^{2}\right)+c\right]^{2}} d x \\
& =\frac{1}{a^{2} \pi} \int_{-\infty}^{\infty} \frac{\left(1+x^{2}\right)^{3-n}}{\left[x^{4}+\frac{2 a+b}{a} x^{2}+\frac{a+b+c}{2 a}\right]^{2}} d x
\end{aligned}
$$

where $n=n^{1 / 2}$. The roots of the denominator are

$$
x= \pm\left[-\left(\frac{b}{2 a}+1\right) \pm \frac{\sqrt{b^{2}-4 a c}}{2 a}\right]^{1 / 2}
$$

with the various combinations of the \pm signals giving four roots. In the present case, $a, b$, and $c$ are greater than zero and $b^{2}>4 a c$. Thus the roots can be written $\pm i R_{1}$ and $\pm i R_{2}$ where

$$
R_{2}=\left[(1+/ 2 a) \pm\left(\sqrt{ } b^{2}-4 a c\right) / 2 a\right]^{1 / 2}=\sqrt{\alpha \pm \beta}
$$


This integral can be evaluated by the residue theorem. There are two poles in the upper half plane, at $i R_{1}$ and $i R_{2}$. The restidues at these poles may be obtained by using Cauchy's theorem. The result is

$$
\begin{aligned}
I_{2 n}=\frac{1}{4 \alpha^{2} \beta^{2}}\{(3-n) & {\left[\frac{(1-\alpha+\beta)^{2-n}}{\sqrt{\alpha-\beta}}+\frac{(1-\alpha-\beta)^{2}-n}{\sqrt{\alpha+\beta}}\right] } \\
& +\left(\frac{\alpha}{\beta}+\frac{3}{2}\right) \frac{(1-\alpha-\beta)^{3-n}}{(\alpha+\beta)^{3 / 2}} \\
& \left.-\left(\frac{\alpha}{\beta}-\frac{3}{2}\right) \frac{(1-\alpha+\beta)^{3-n}}{(\alpha-\beta)^{3 / 2}}\right\}
\end{aligned}
$$

This completes the details needed for the application of the three-level model of the polarizability to DFWM in $\mathrm{KCl}: \mathrm{KReO}_{4}$. 


\section{PUBLICATIONS:}

1. M.H. Wilkening and D. E. Watkins, "Air exchange and $222 \mathrm{Rn}$ concentrations in the Carlsbad Caverns," Health Physics 31, $139(1976)$.

2. R. K. Ahrenkiel, P. Weiss, D. E. Watkins, S. K. Gulati, and W. W. Grunneman, "The Faraday effect in $\mathrm{Hg} 1-{ }^{-} \mathrm{Cd}_{x} \mathrm{Te}$ at $\mathrm{CO}_{2}$ laser wavelengths," J. App 1. Phys. 49, 2265 (1978).

3. C.R. Phipps, Jr., S. J. Thomas and D. E. Watkins "Effect of nonlinear refraction on beam brightness in laser fusion applications," Proceedings of the International Conference on Lasers 179, STS Press, Alexandria, VA.

4. D. E. Watkins, J. F. Figueira and S. J. Thomas, "observation of resonantly enhanced degenerate four-wave mixing in doped alkali halides," Opt. Lett. 5, $169(1980)$.

5. D. E. Watkins, C. R. Phipps, Jr. and S. J. Thomas, "The determination of the third order nonlinear optical coefficients of germanium via ellipse rotation," Opt. Lett. 5,248 (1980).

6. D. E. Watkins, C. R. Phipps, Jr. and S. J. Thomas, "Observation of amplified reflection via degenerate four-wave mixing at $\mathrm{CO}_{2}$ laser wavelengths in germanium," Opt. Lett. 6,76 (1981). 